\title{
Requested Documentation Associated with Soil Sampling at Building 419
}

\author{
March 12, 2012
}

V.Salvo 
This work performed under the auspices of the U.S. Department of Energy by Lawrence Livermore National Laboratory under Contract DE-AC52-07NA27344. 


\section{Building 419 Map Showing Soil Sample Locations}




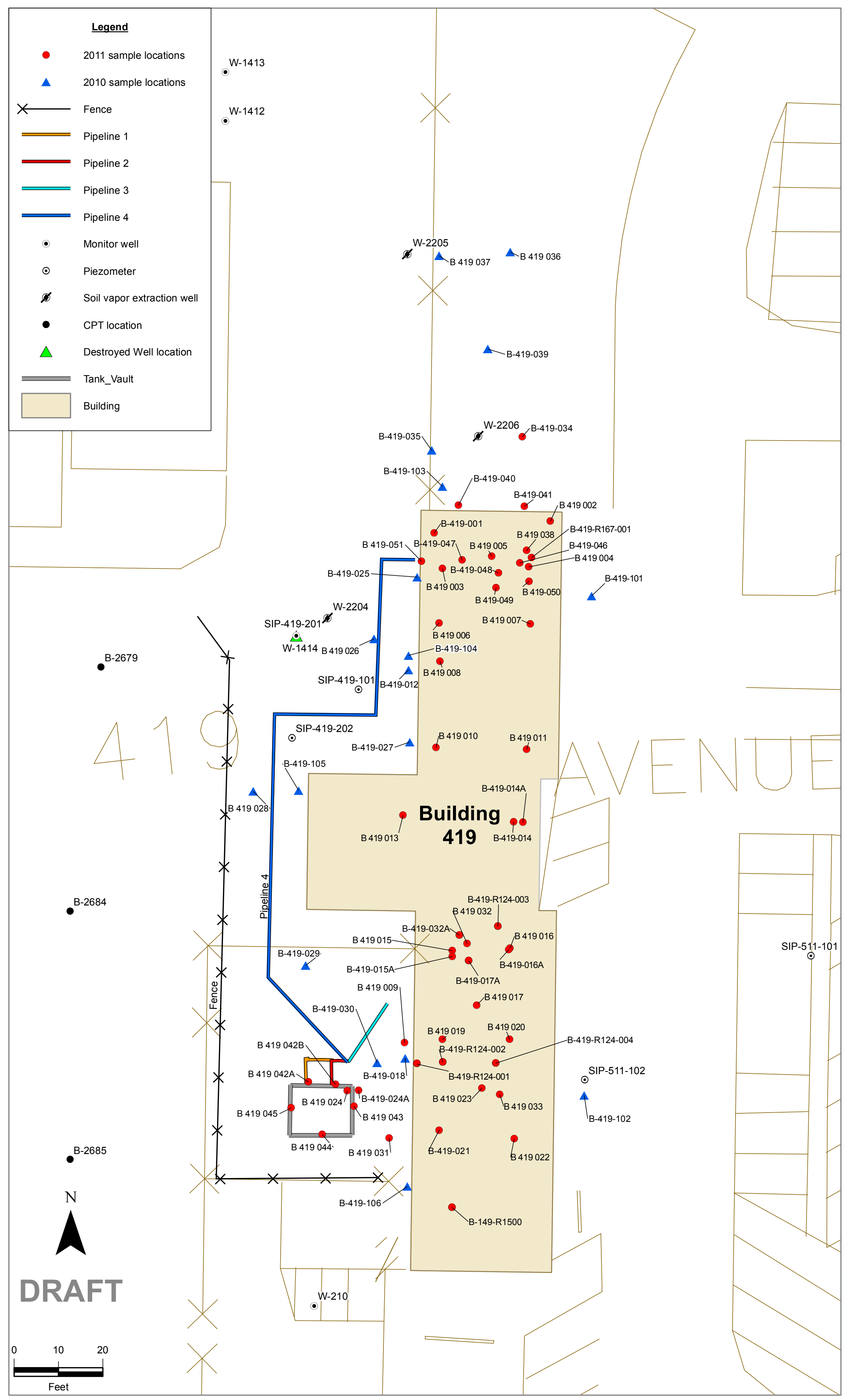

Z:|projectspacels200ls200hydrogeol2011/SitewidelB419_ClosurelPhase_II_data_analysislgis|B419SampleLocs.mxd 20120214 


\section{Logs for Building 419 Borehole Locations 40 and 41}


Borehole/Well Construction Log

Page 1 of 6

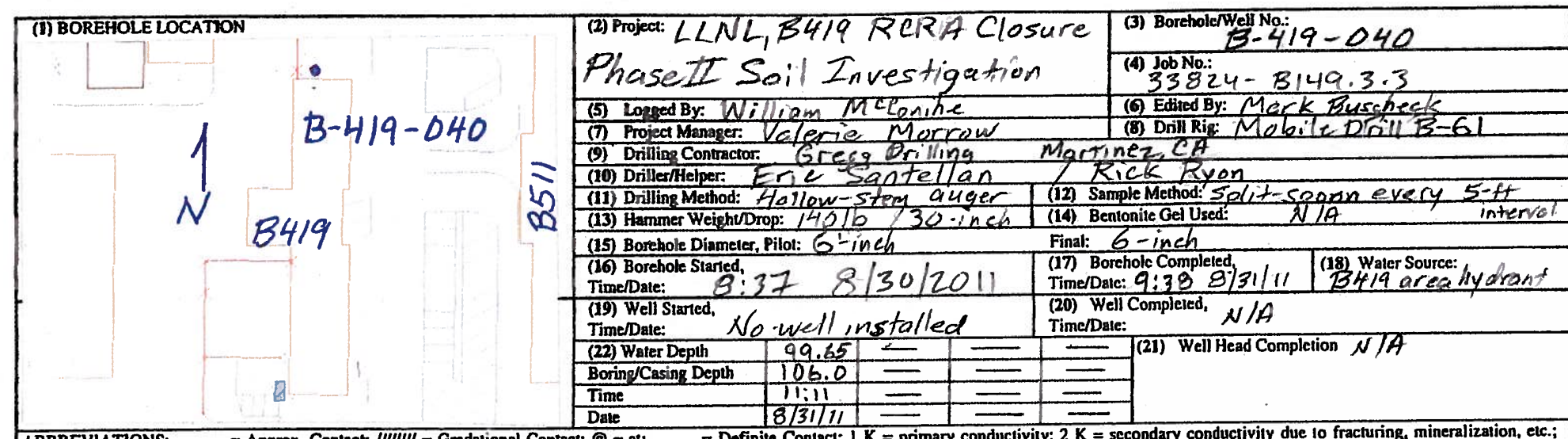

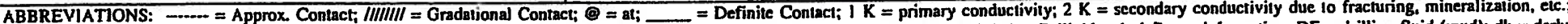

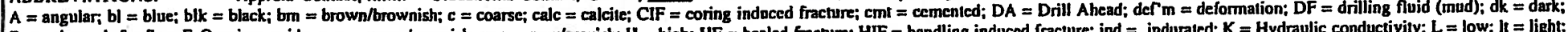

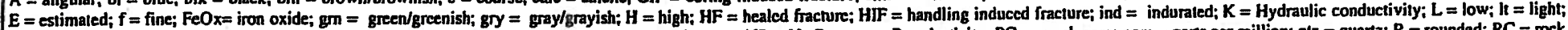

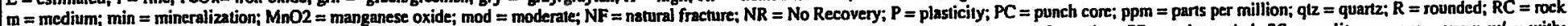

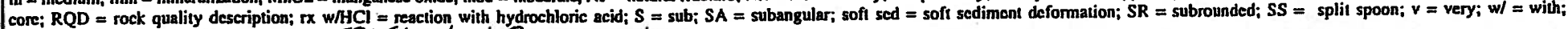
$x$-beds $=$ cross beds; $y \mid w=$ yellowlyellowish. $S P:$ Standard Penetrometer

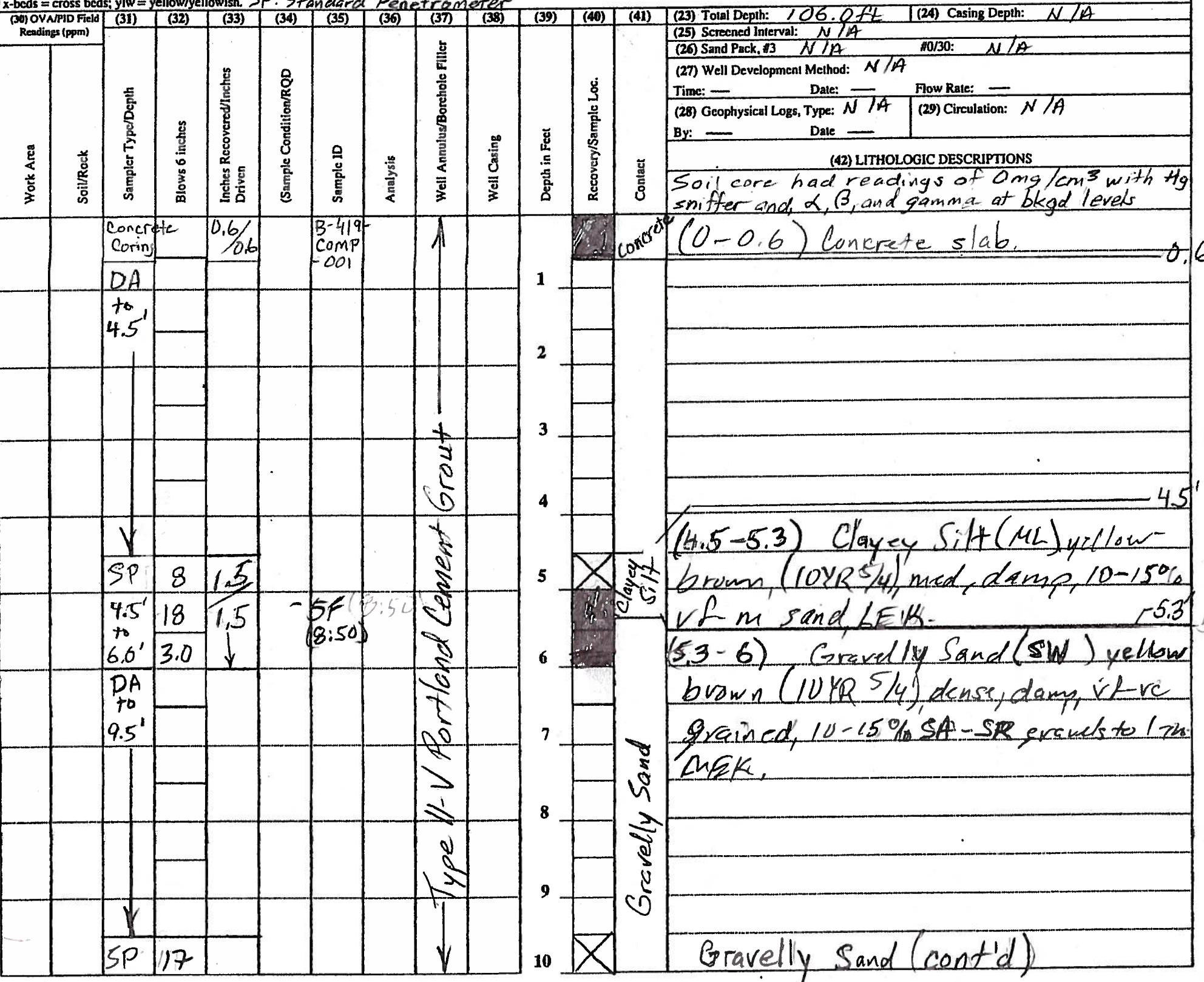

Note: Numbers 1-42 listed on this log correspond to Subsections 6.4.1-6.4.42 of SOP 1.1. 
Borehole/Well Construction Log (cont) Page 2 of 6

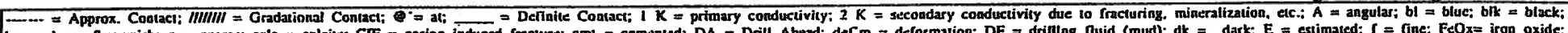

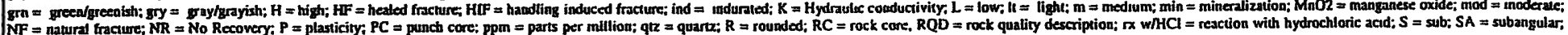

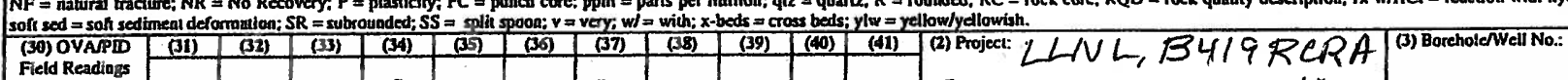

\begin{tabular}{|c|c|c|c|c|c|c|c|c|c|c|c|c|c|c|}
\hline \multicolumn{2}{|c|}{ 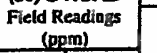 } & \multirow{4}{*}{ 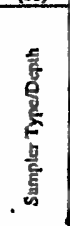 } & \multirow{4}{*}{ 毫 } & \multirow{4}{*}{ 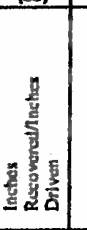 } & \multirow{4}{*}{ 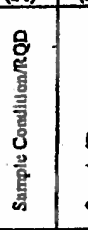 } & \multirow{4}{*}{\multicolumn{2}{|c|}{ 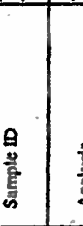 }} & & \multirow{4}{*}{ 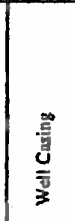 } & \multirow{4}{*}{\multicolumn{2}{|c|}{ 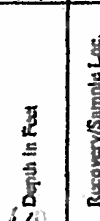 }} & & \multirow{3}{*}{$\begin{array}{l}\text { Closure Phase II } \\
\text { Soil Investigation }\end{array}$} & \multirow{4}{*}{$B-419-040$} \\
\hline & & & & & & & & & & & & & & \\
\hline$\xi$ & & & & & & & & & & & & & & \\
\hline$\frac{\sigma^{\circ}}{3}$ & 害 & & & & & & & & & & & & Noles: & \\
\hline & & $\begin{array}{l}9.5 \\
+4 \\
11.0^{\circ}\end{array}$ & $\frac{19}{25}$ & 14 & & $\mid \begin{array}{l}100 \\
0: 05\end{array}$ & & 91 & & & & है & $30-40 \% S A-5 R$ & 15 \\
\hline & & $\begin{array}{ll}D A \\
t,\end{array}$ & & & & & & & & & & $\overrightarrow{\bar{y}}$ & & \\
\hline & & 145 & & & & & & & & . & & & & \\
\hline
\end{tabular}

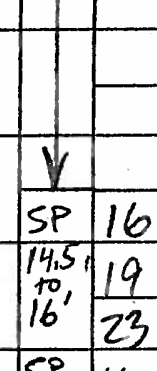

159,14418

to 17

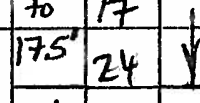

$D A$

$17,5^{+}$

19.5

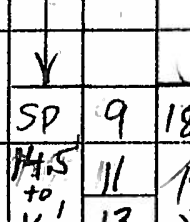

\begin{tabular}{llll}
16 & 13 & 18 \\
\hline
\end{tabular}

$-20 F$

$(9: 2.4)$

DA,

to

245

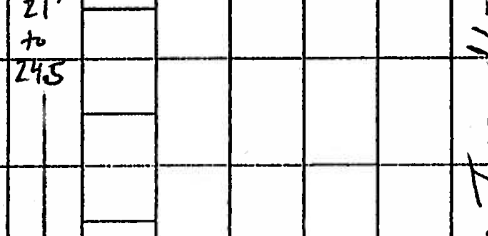

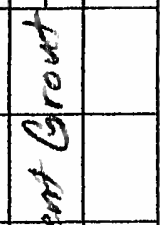

16

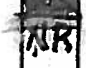

तर

so tol-ike, $20-30 \%$ Vf-ve sand, MEK

$(163-17.5)$ Silty sand (SM), light olive brow $\$ \rightarrow(2+5.5 / 4)$, med, damp, if-m grained, $10-20 \%$ $\eta^{5}$ fines, $L-M E K$.

18

齐

$(14,5$ - 163) Scudy Gravel (GP ) Yollew brown $\left(104 R^{6} / 4\right)$, dense, damp, SA-5R grawe

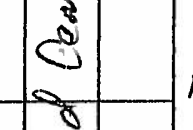

s

o

$>$

西

$>$

\&

ti
-25 F

$(9.42)$
20

$\pm(195-21)$ Clayey Silt (ML) light olive brown ain $(2.5 Y 5 / 6)$, ned dom, trace of - re sand, $\vec{s}$ Heght, low p lasticity, LEK.$$
21 \text {. Hoght, low p lasticity, LEK. }
$$

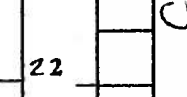

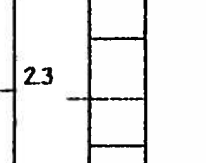

24

25

$(24.5-26)$ Gaud, (SW), Light olve brown, $(2.5)$ $5 / 6)$, med, damp, $v f$-ve grained, trace fines, $M E K$

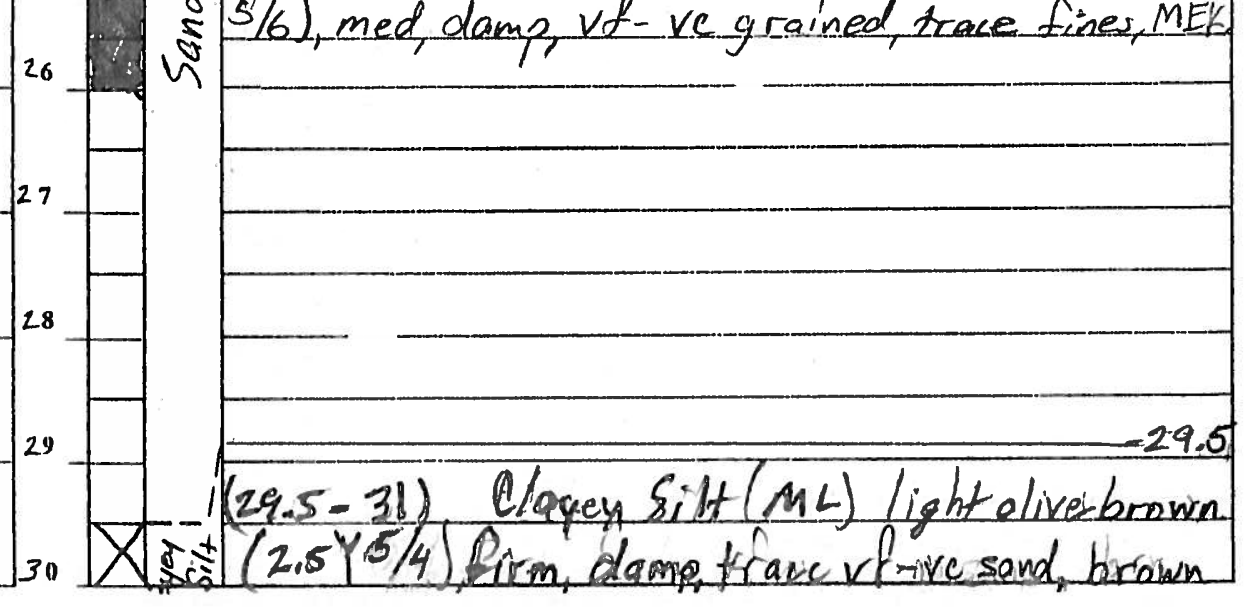


Borehole/Well Construction Log (cont) Page 3 of 6

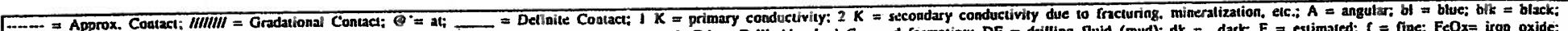

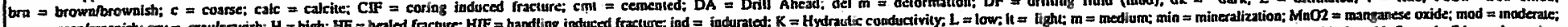

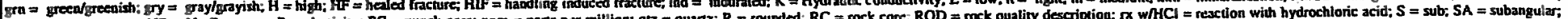

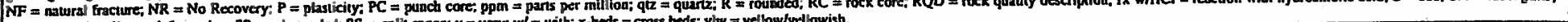

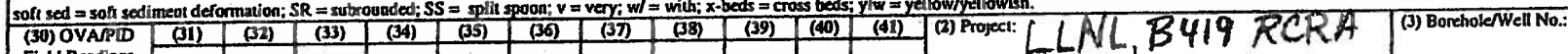

\begin{tabular}{|c|c|c|c|c|c|c|c|c|c|c|c|c|c|}
\hline \multirow{2}{*}{\multicolumn{2}{|c|}{ 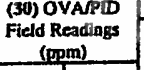 }} & \multirow{4}{*}{ 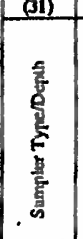 } & \multirow{4}{*}{\multicolumn{2}{|c|}{ 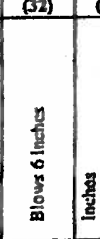 }} & \multirow{4}{*}{\multicolumn{2}{|c|}{ 等) }} & \multirow{4}{*}{ 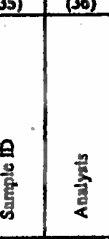 } & \multirow{4}{*}{ 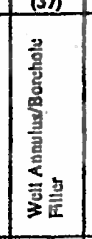 } & \multirow{4}{*}{\multicolumn{2}{|c|}{ 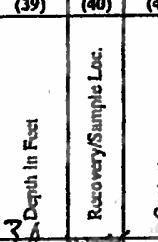 }} & \multirow{3}{*}{\multicolumn{2}{|c|}{$\begin{array}{l}\text { Closure Phase II } \\
\text { Soil Investigation }\end{array}$}} & \multirow{3}{*}{$B-419-0.40$} \\
\hline & & & & \multirow{3}{*}{ | } & & & & & & & & & \\
\hline$\xi$ & & & & & & & & & & & & & \\
\hline 羊 & 褰 & & & & & & & & & & $\frac{0^{2}}{3}$ & Noles: & \\
\hline & & $\begin{array}{l}29.5 \\
+0 \\
t 0\end{array}$ & 24 & & & 30100 & & $\pi$ & & & 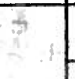 & caliche LEK & \\
\hline & & $D A$ & & $D$ & & & & & & & & & \\
\hline & & $34,5^{\prime}$ & & & & & & & 32 & & & & \\
\hline & & & & & & & & & & & & & \\
\hline & & & & & & & & & 33 & & $x$ & & \\
\hline & & ( & & & & & & & & & & & \\
\hline & & & & & & & & & 34 & & it & & \\
\hline & & V & & & & & & & & & $\lambda$ & & \\
\hline & & SP & 17 & $B$ & & & & & 35 & $x$ & 8 & 234.5-36ft, same & as a bove, shiff, tight \\
\hline & & 然 & 18 & $\mid 18$ & & $\begin{array}{l}35 \mathrm{H} \\
100 \mathrm{O}, 1\end{array}$ & & & & & (a) & cream eolbred calich & \\
\hline & & $\mid \frac{36}{D A}$ & 26 & $y$ & & & & & 36 & & J & & \\
\hline & & 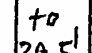 & & & & & & $\overrightarrow{0}$ & & & & & \\
\hline & & 39,5 & & & & & & t & 37 & & & & \\
\hline & & & & & & & & 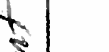 & & & & & \\
\hline & & & & & & & & है & 38 & & & & \\
\hline & & & & & & & & 8 & & & & & \\
\hline & & & & & & & & & 39 & & & & \\
\hline & & r & & & & & & of & & & & 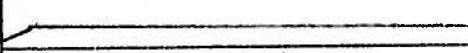 & 39.5 \\
\hline & & sp & 16 & 19 & & & $x$ & 5 & 40 & X & & $(39,5-41,0)$ Sandy & Silt, (ML) olive yellow, \\
\hline & & $\begin{array}{l}393 \\
50 \\
41\end{array}$ & $\frac{15}{13}$ & $1 \varepsilon$ & & {$[0: 18\}$} & Q & 0 & & & & $\begin{array}{l}(2.5 y \text { y } / 4) \text {, snifl, dicy } \\
\text { cilored caliche, blk }\end{array}$ & $\frac{10-13 \% \text { of }-f \text { sand eream }}{1 n-0 \text { stainsto } 8,25 \text {-ench }}$ \\
\hline & & $D A$ & & & & & & $\Delta$ & & & & LEK & \\
\hline & & $\left|\begin{array}{ll}+\infty \\
44,5^{2}\end{array}\right|$ & & & & & & & 42 & & & & \\
\hline & & & & & & & & & & & & & \\
\hline & & & & & & & & 8 & 43 & & t & & \\
\hline & & & & & & & & 3 & & & $\dot{n}$ & & \\
\hline & & & & & & & & & 44 & & $\lambda$ & & \\
\hline & & 11 & & & & & & & & & & & \\
\hline & & SP & 18 & 19 & & & & & 45 & Xi & 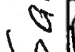 & $(44,5-46,0)$ Sendys & it (ML) yellow $6 \mathrm{~mm}$ \\
\hline & & $\begin{array}{c}44,5 \\
\text { to }\end{array}$ & 19 & 19 & & $\begin{array}{l}4557 \\
10: 29)\end{array}$ & & & & & $v$ & $(10 Y R 5 / 4)$, med, dry & $20-30 \%$ vf-m sand \\
\hline & & $46^{\prime}$ & 31 & $\psi$ & & & & & 46 & & & $10=10$ SA gravels ti & 0.75 inch, ${ }_{1}$ ight, LEK. \\
\hline & & $\left|\begin{array}{ll}D A \\
P A\end{array}\right|$ & & & & & & & & & & & \\
\hline & & 49,5 & & & & & & & 47 & & & & \\
\hline & & & & & & & & & & & & & \\
\hline & & & & & & & & & 48 & & & & \\
\hline & & & & & & & & & & & & & \\
\hline & & & & & & & & & 49 & & & & $-49.5^{\prime}$ \\
\hline & & \pm & & & & & & & & 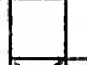 & - & $(49.5-51.0)$ Sendy & Bravelf (GM/sW) yello \\
\hline & & SP & 22 & $18 / 19$ & & & & & 0 & 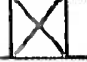 & & {$\left[\left(10 Y R^{5} 5 / 4\right)\right.$ dense-h } & $S A S R$ \\
\hline
\end{tabular}


Borehole/Well Construction Log (cont.) Page 4 of 6

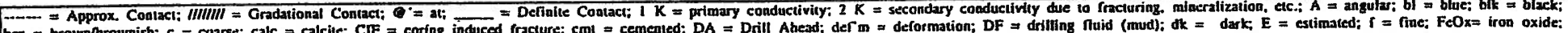

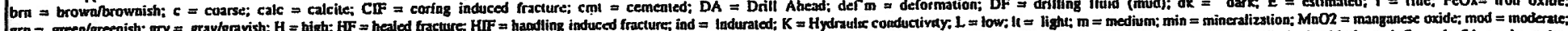

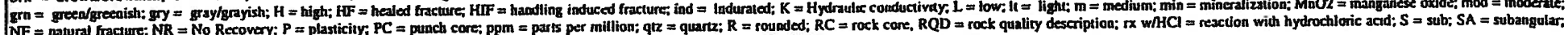

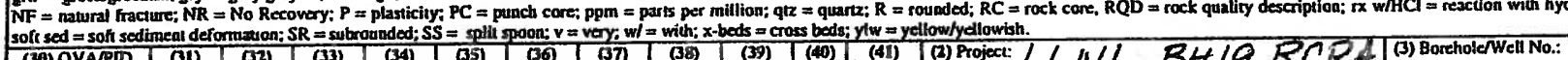

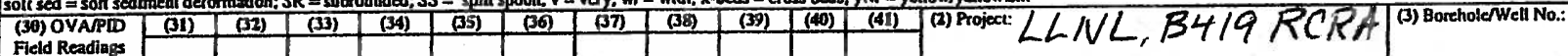

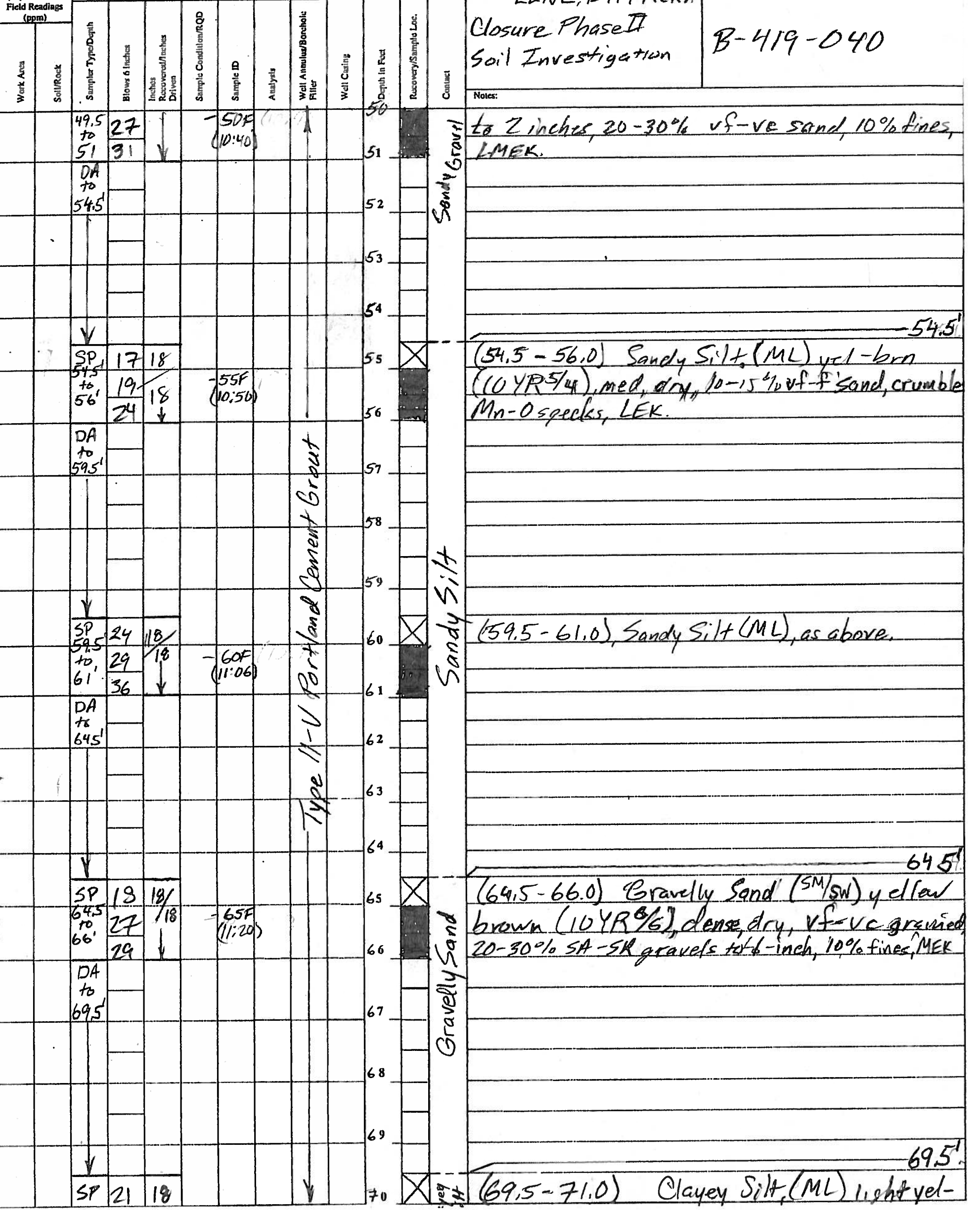


Borehole/Well Construction Log (cont.) Page 5 of 6

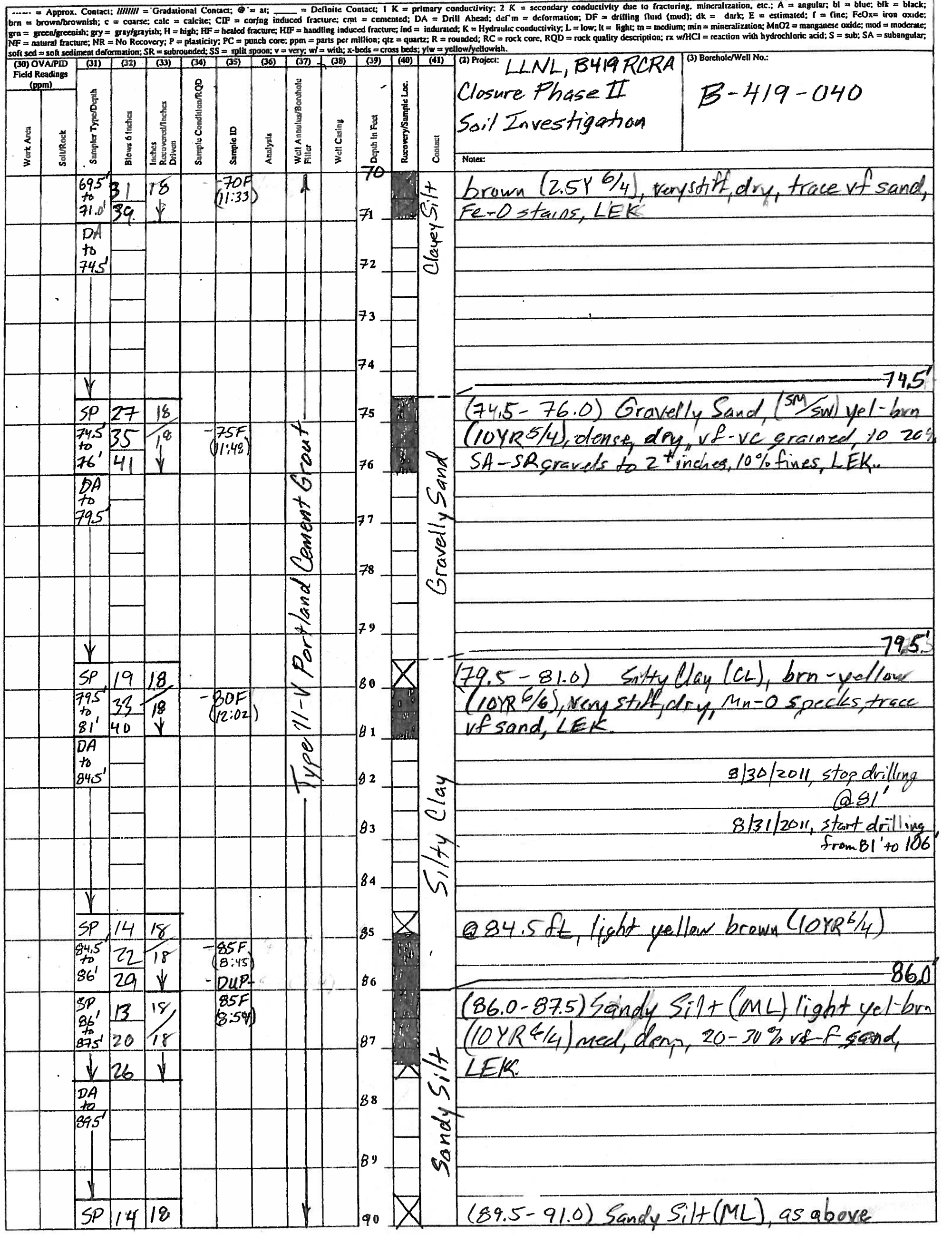


Borehole/Well Construction Log (cont.) Page 6 of 6

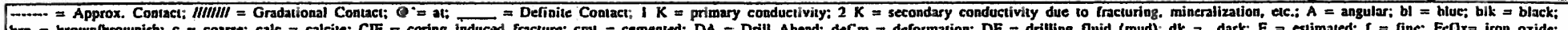

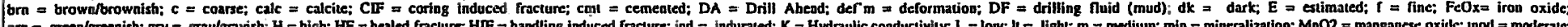

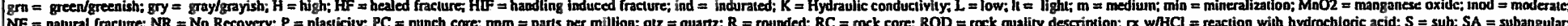

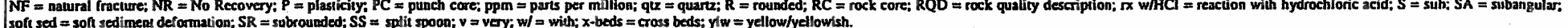

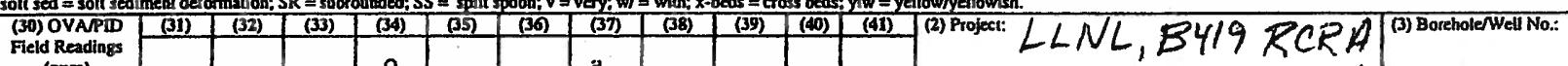

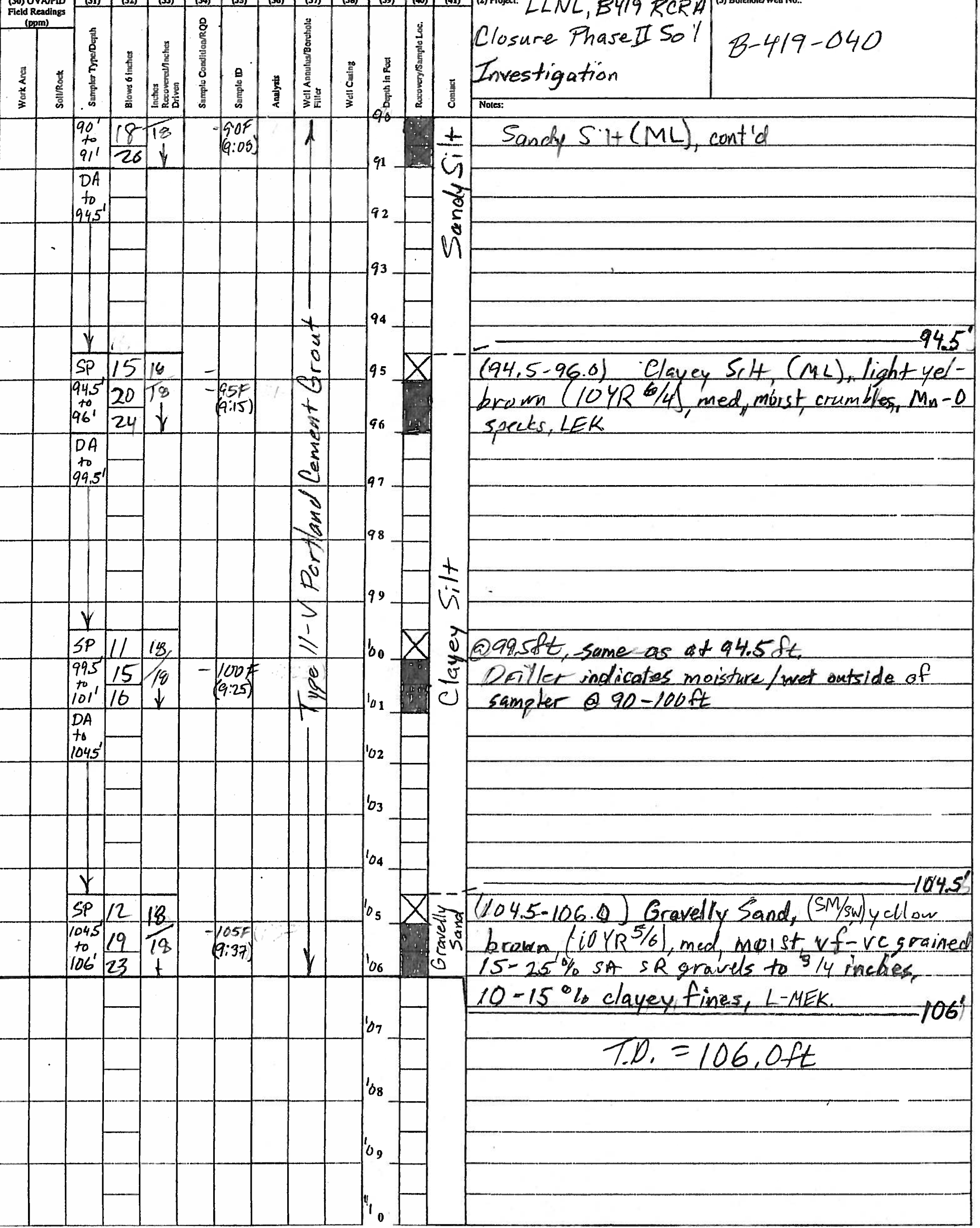


Borehole/Well Construction Log

Page 1 of 6

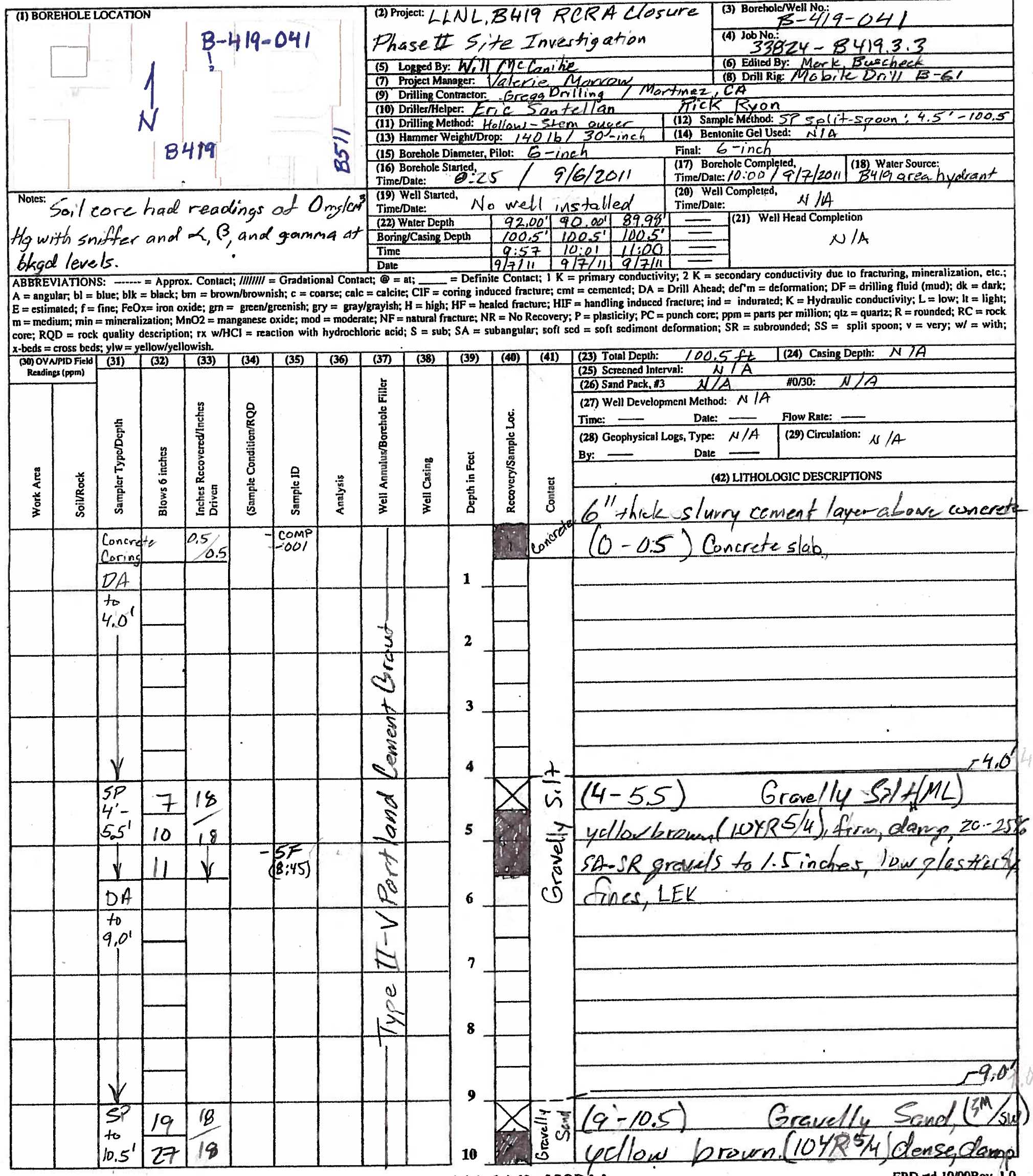

Note: Numbers 1-42 listed on this log correspond to Subsections 6.4.1-6.4.42 of SOP 1.1. 
Borehole/Well Construction Log (cont.) Page 2 of 6

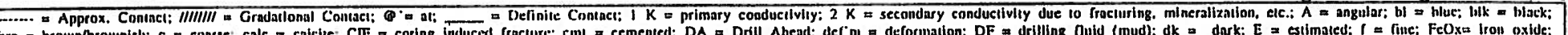

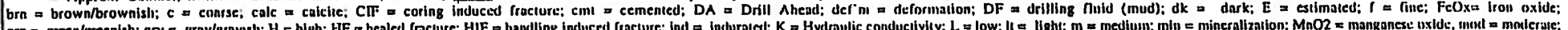

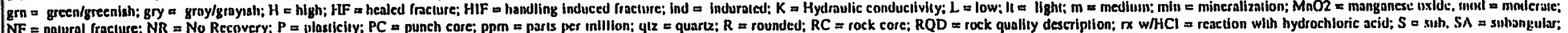

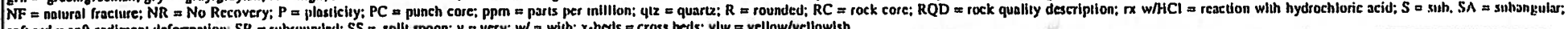

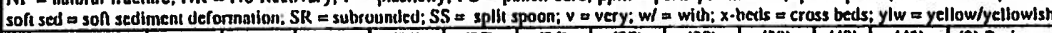

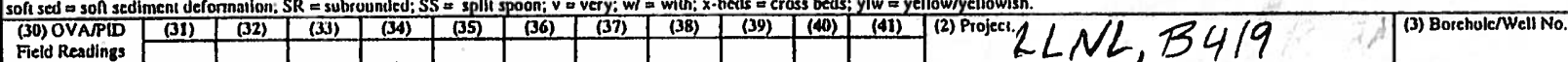

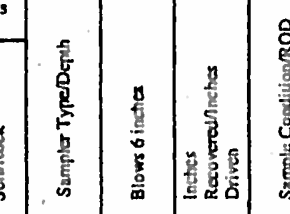

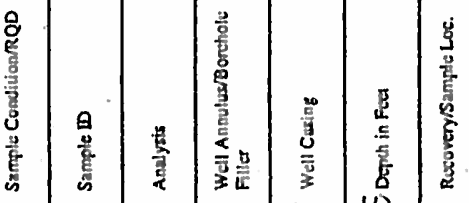

RCRA Closure Phasell

B $-419-041$

营

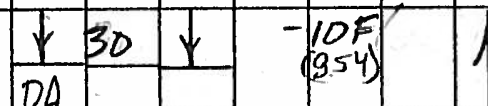

to $14.0^{\prime}$

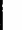

SP 13

$15,522-18$

1264

$D A$

to

$19^{\prime}$

4

\begin{tabular}{l|l|l}
\hline$p$ & 14 & 18 \\
\hline 19 &
\end{tabular}

$20.519 / 15$

\begin{tabular}{c|c|c|}
1 & 20 & $y$ \\
\hline 58 & 15 & 18
\end{tabular}

$\begin{array}{r}-20 \\ 9: 10 \\ - \text { Dup. } \\ \hline\end{array}$

\begin{tabular}{l|ll}
$5 P$ & 15 & 18
\end{tabular}

\begin{tabular}{c|c|c}
205 & 21 \\
-22 & 21 & 18
\end{tabular}

$\forall 27$

DA

to 24

$\uparrow$

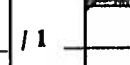

Site Investigation

so

12

8

if-ve grained $25-35 \%$ gravels tar $3 / 4=$ inch $10 \%$ finus, LEK - $M$.

仓े

13

$\geq$

0
0
0
0

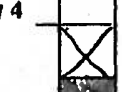

$(14-15.5)$, same ar at 9-10.5ft.

15

16

(9:05)

4

\begin{tabular}{l|l|l|l|}
$5 P$ & 13 & 18 \\
\hline
\end{tabular}

$2 5 5 \longdiv { 1 7 }$

189

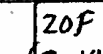

$(9: 18)$

DA

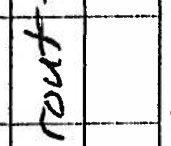

is

0

at

18

cy

7

19

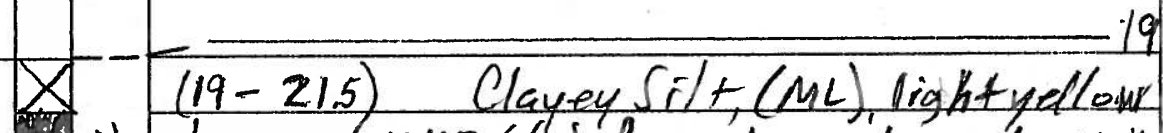

20 * $*$ brown $(104 R 6 / 4$ ) firm, dom, low plartienty

af

i LEK

21

3

$>$

$2^{2}$

$$
\text { है। }
$$

23

in

24

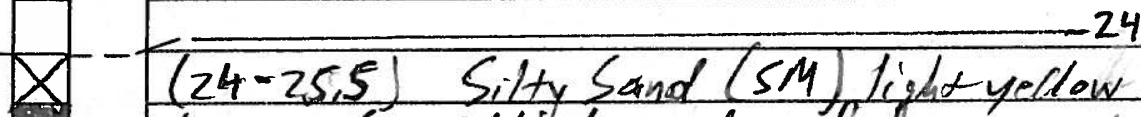

25 - o brown, (10KR 6/4) fouse, dry, vf-f grained, vitu-150\% silty fines L-NEK.

to 29

26

r

in

27

1

28

29

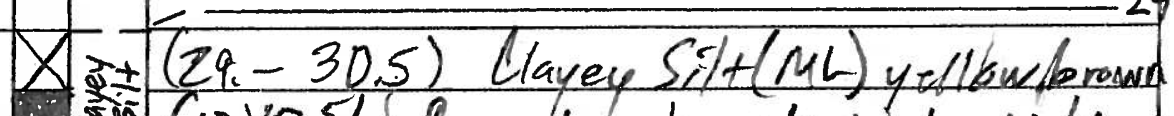


Borehole/Well Construction Log (cont.) Page 3 of 6

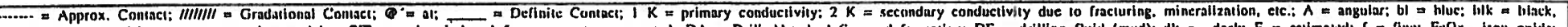

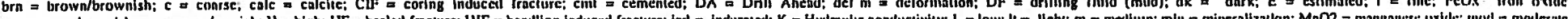

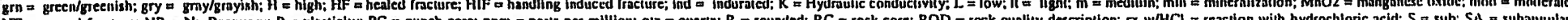

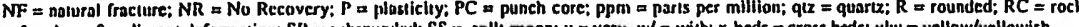

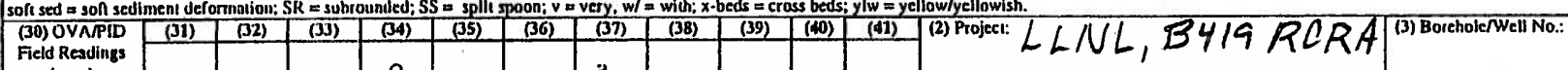
(ppm)

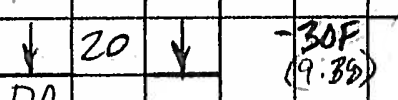

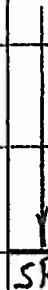

$5 P_{31} 11$

$34 ! \frac{17}{15}$

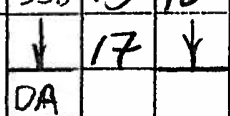

$-35 F$

$(9.50)$

$39^{\prime}$

1

$\begin{array}{llll}59 & 18 & 18\end{array}$

$\begin{array}{lll}39^{\prime} & 18 & \\ 405^{\prime} & 22 & 18\end{array}$

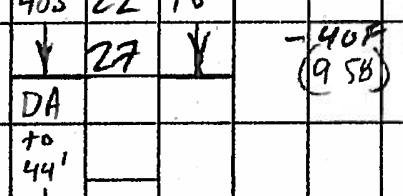

\section{位}

1

\section{3}

31

Closure PhaseII Soil

$B-419-041$

\section{Investigation}

trace $S R$ gravels to $1 / 2$ inch, $L E K$.

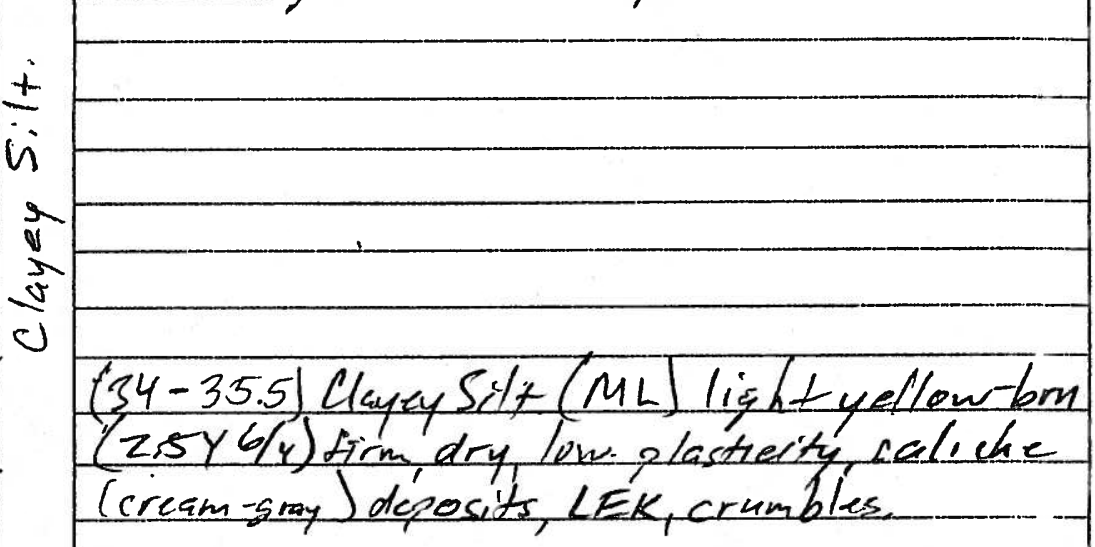
$i$ brown $\left(2.5 y^{5} / 6\right)$, firm, damp $15-25^{\circ}$ orf sand \&- crumbles, some plasticily, LEK,

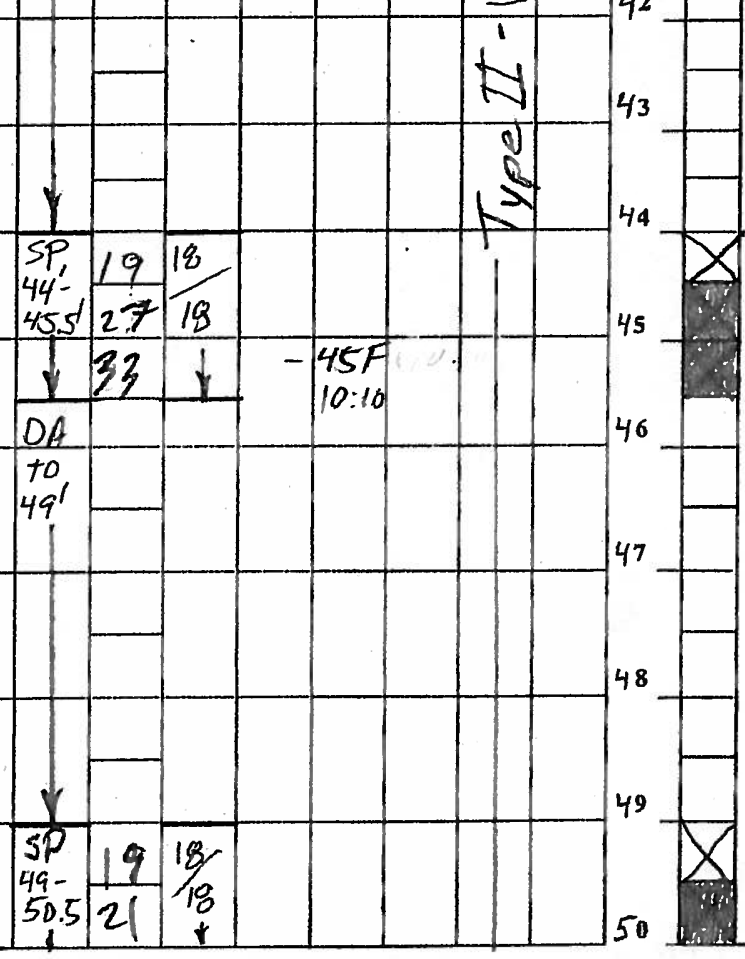
n

$(44-45.5)$ Gravelly Sand, (5M/sw) yed-6m $\left(10 Y R^{5} 6\right)$, dense, damp of-vc grained, $20-30 \%$ $S A-S R$ gravels to 1.5 incher, $10 \%$ finer, L-MEK.

$(49 .-50.5)$ Same as at 44-45.5ft, graved up to Zinches. 
Borehole/Well Construction Log (cont.) Page 4 of 6

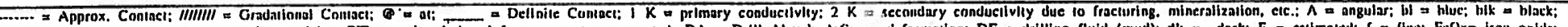

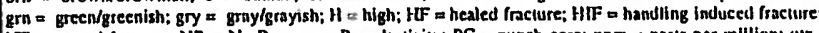

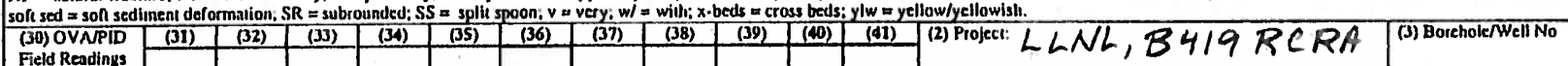

Closure Phaselt Soil

$B-4 / 9-041$

$$
\begin{array}{lll}
1 & 32 & 1 \\
\hline D A & &
\end{array}
$$

DA Investigation

\section{(1)}

SP 15

1518

65.52218 $y 23 y$ $D A 22$

to

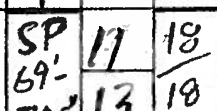

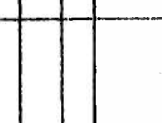
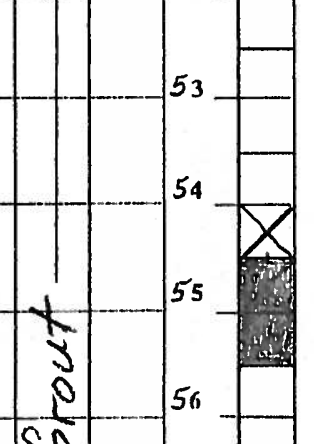

$-57$

60 .

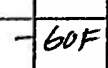

อัo

5

4

4
0

A
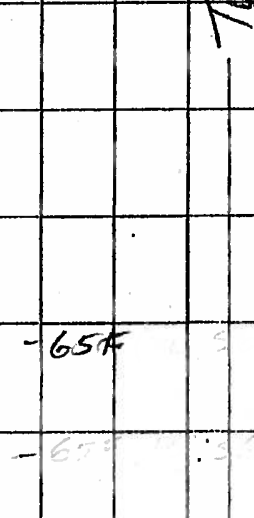$$
52
$$

54 55

58

59

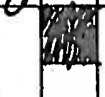

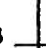

61

$62+$

63

64

区

65

66

67

68

69

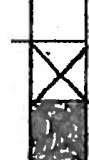

Gravelly Sand ( $S M / S w)$, cont'd

QS4ft, same as above with $35-40 \%$ SA-SR2 grands to 1.5 inch.

Q.54 ft, Gravelly sand, as above, $15-25 \%$ SAsor eravels to $3 / 4$-inch.

(5)

Q64ft. Gravelly Saud os above

9/6/201 Stop@10'

$(69-70.1)$ same as ebove, inth 10-20\% $S A-S R$ grovele to 1.5 inath. 
Borehole/Well Construction Log (cont.) Page $\$$ of 6

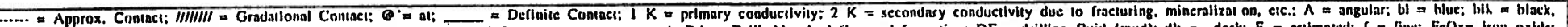

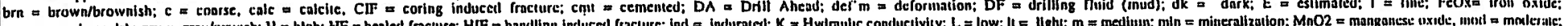

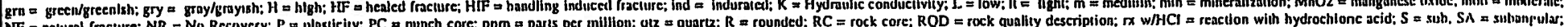

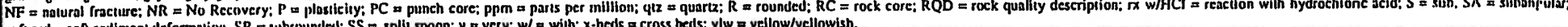

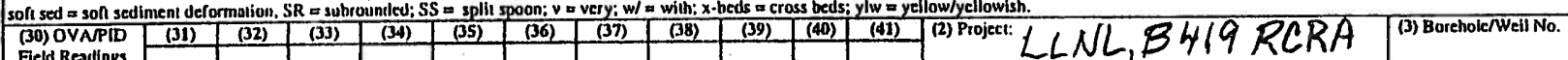

, Closure Phase II Soil

Investigation

$$
B-419-041
$$

Noles: $(70.1-70.5) C l a y=y ~ S i l t(M L)$ yellow brown

$54^{5}-16 \quad 18$

$75.518-18$

$\downarrow 20 *-75 F$

$D A$

to

$79^{\prime}$

(9:19)

SP 1918

30,1318

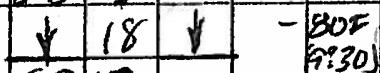

SP $1318=D$ MP -

$80.5^{\prime}$

$-82^{\prime} 16$

1
1

805

$D A$

to 84

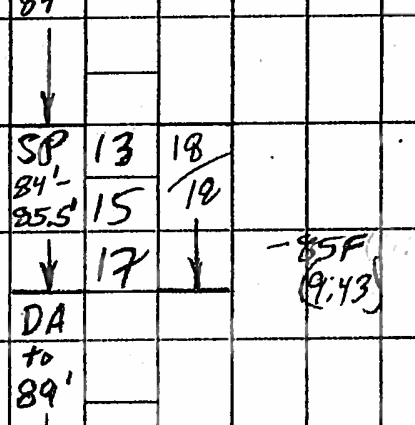
$(10 Y R 6 / 5)$, ght dry, tight, fracem sand, $m_{n}=0$. specks, LEK.

$(7,4-75.5)$ Clayey $S 1+(M L)$, brown-yellow

71

72

73

74

76

77

78

$\frac{6}{15}$

$x+8$

$\mathrm{A}^{\circ}$

iे

3

rit

83

83

0 in

$+279-80,5)$ Clayey Silt $(M L)$ as abave.

\section{loYR Ela), firon, dry,
Cow plasticity, LEK}

$(84-05.5)$ Clayey Silt (ML)/ightyelleut brown $(250 y)$ G firm, damp, lourplasticity LEK. 
Borehole/Well Construction Log (cont.) Page 6 of 6

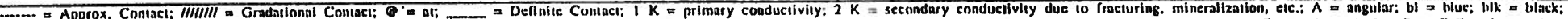

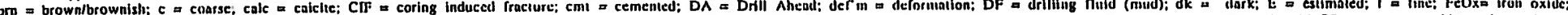

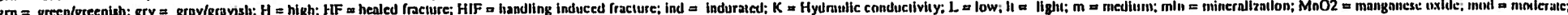

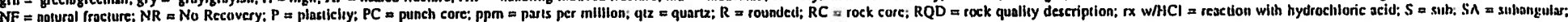

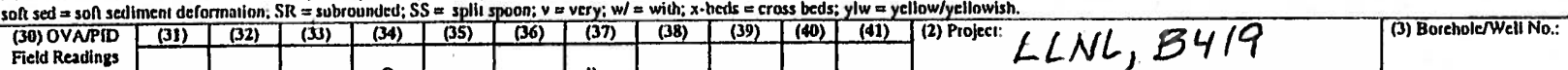

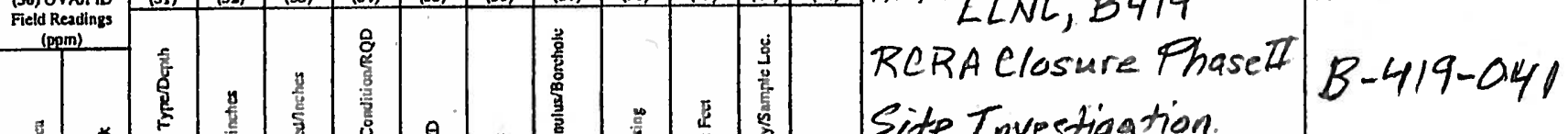

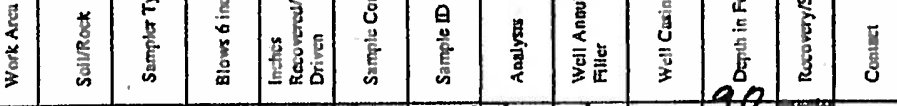

$116 \backslash$ 90F 190

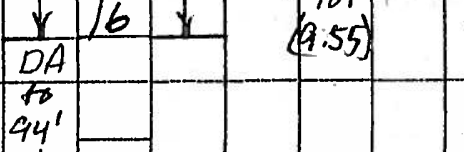

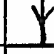

\begin{tabular}{l|l|l|}
$5 p$ & 11 & 18 \\
94 & 18 \\
$955^{\prime}$ & 13 & 18 \\
\hline
\end{tabular}

\begin{tabular}{|c|c|}
\hline 1 & 19 \\
\hline
\end{tabular}

$D A$

99

$91+$

to $1 / 2$ inch, LEK.

92

93

i

ปิ่

$\frac{v}{v}$

$(94-95,5)$ silt (ML) light brewn-yellow
$\left(2,5 y^{6 / 4}\right)$, firm, moist, low plasticity, LEK

96

$97-\frac{x}{6}$

98

99

Sp, 918

$199^{\prime}-\frac{9}{17}$

$\downarrow 20 \sqrt{100 F_{10} 245}$

60

'b 1

$\mathrm{O}_{2}$

$b_{3}$

$b_{4}$

$b_{5}$

$b_{6}$

$b_{7}$

$b_{8}$

tog

$1 \%$
7 (99-100.5) Clayey Silt (ML) light - alive brown. svi $(2.5 \% 5 / 4)$, firm, moist, tight, low plastierity, o Mn-O specks, LEKi

$$
T . D .=100.5 \mathrm{ft}
$$


Building 419 Soil Sample Results 
US EPA

Preliminary Bldg

Industrial Screening

Field Location

Sample Date Sample Identifcation

Matrix Depth Analyte Result Units Flag Level (mg/kg)

19 Background background

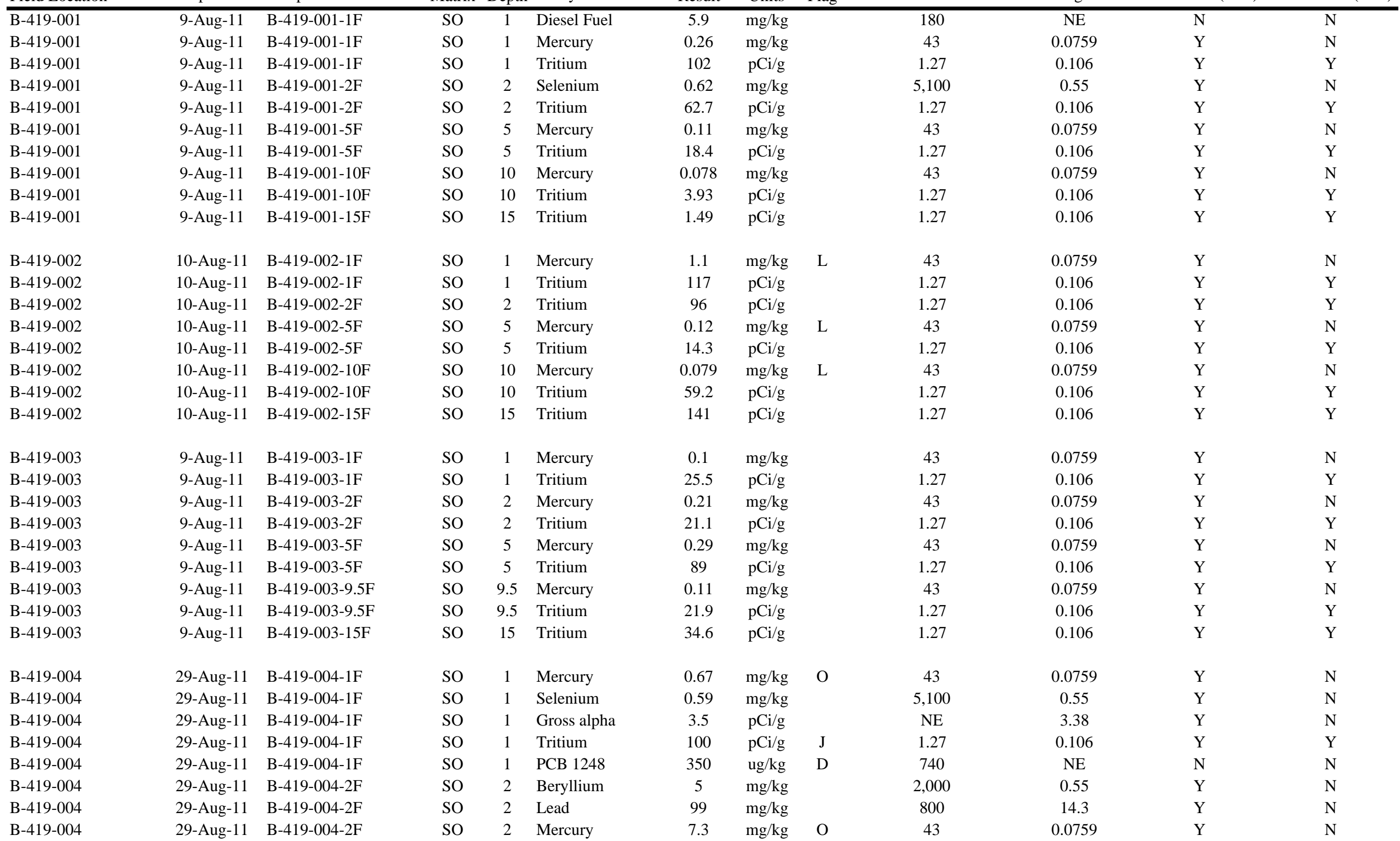


US EPA

Preliminary Bldg

Industrial Screening

Level (mg/kg)

19 Background background

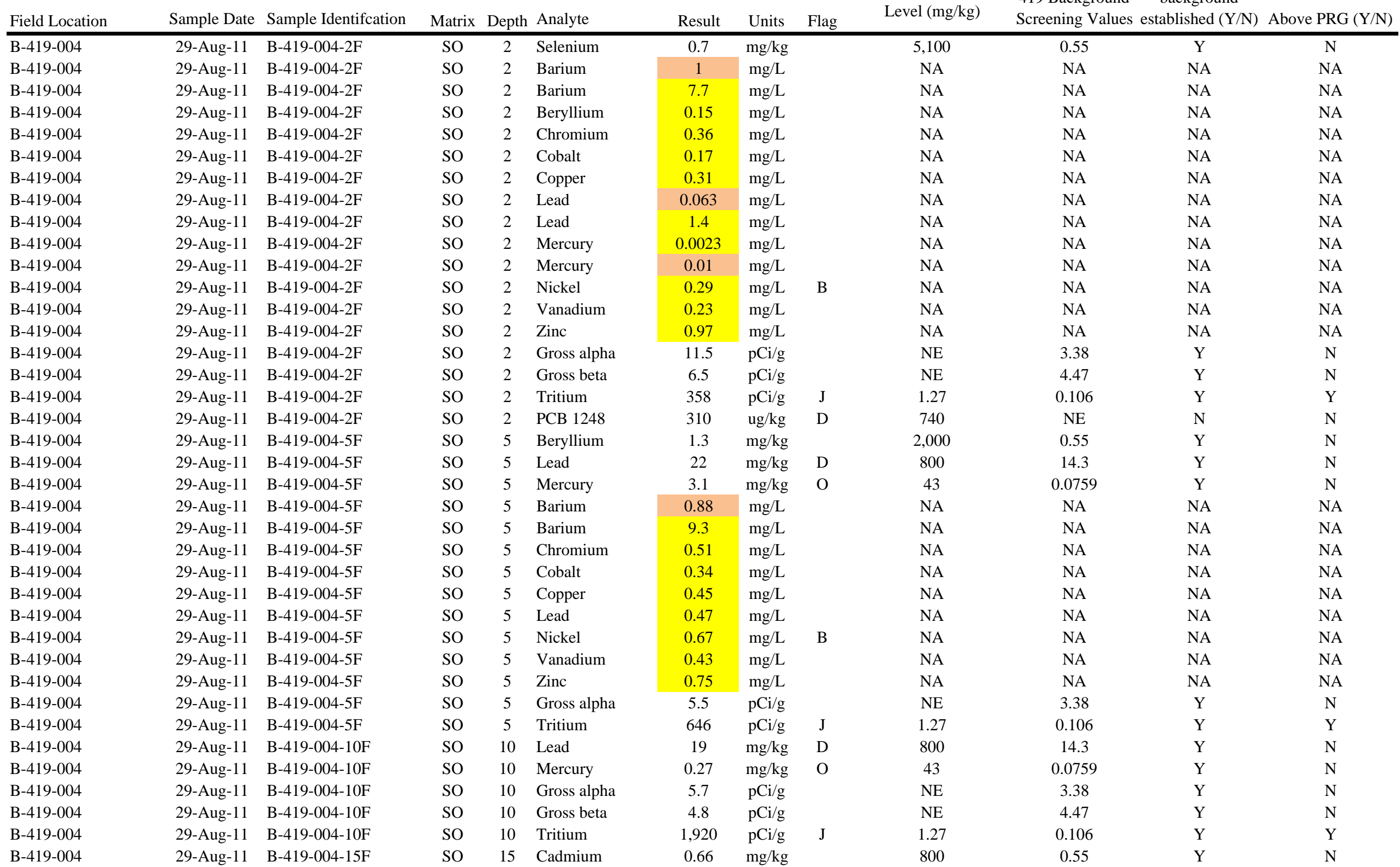


US EPA

Preliminary Bldg

Industrial Screening

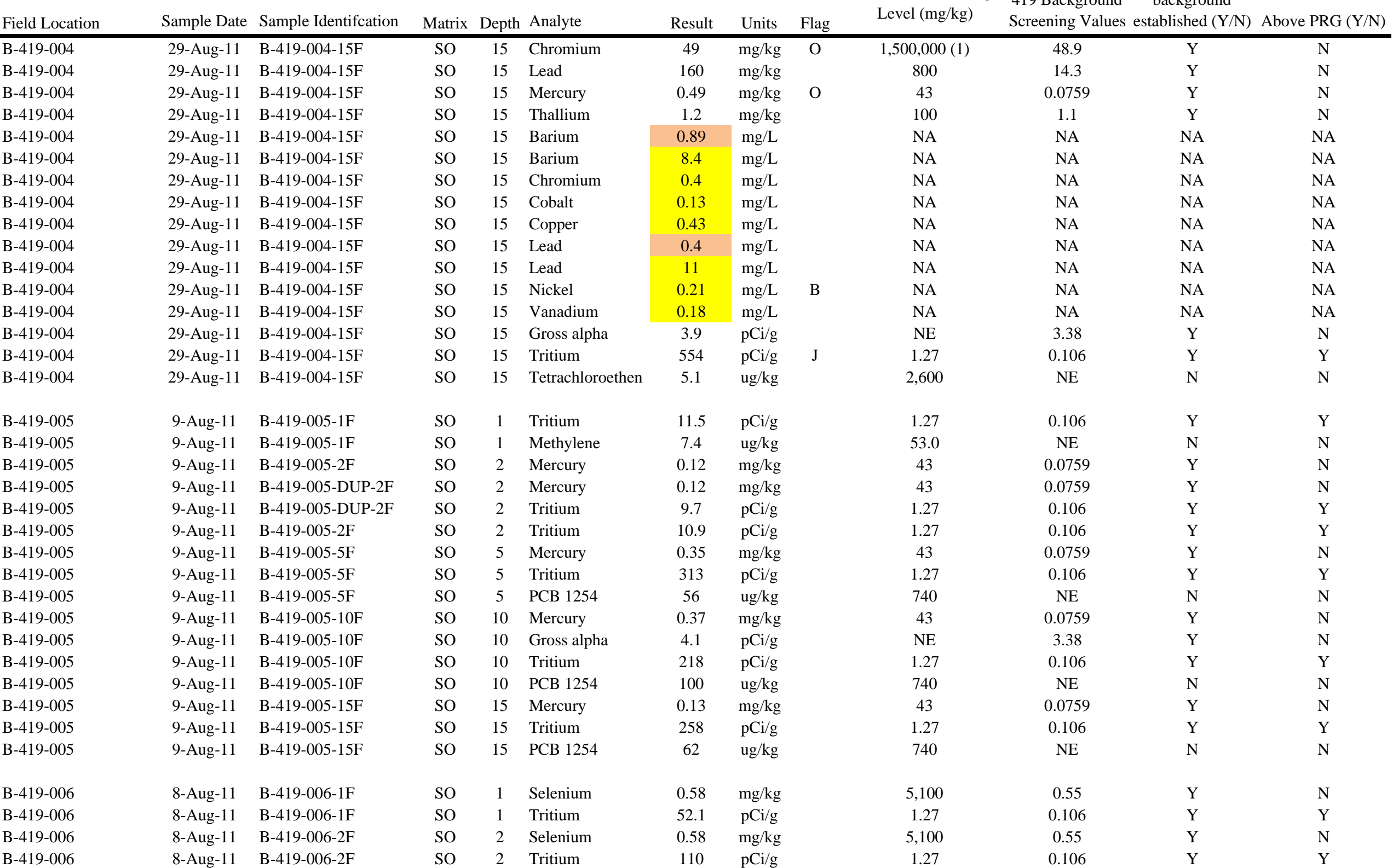


US EPA

Preliminary Bldg

Industrial Screening

Field Location Sample Date Sample Identifcation Matrix Depth Analyte

Result Units Flag

Level (mg/kg)

8-Aug-11 $\quad$ B-419-006-5F $\quad$ SO 5 Thallium

8-Aug-11 B-419-006-5F

8-Aug-11 B-419-006-10F

8-Aug-11 B-419-006-10F

8-Aug-11 B-419-006-15F

8-Aug-11 B-419-006-15F

8-Aug-11 B-419-007-1F

8-Aug-11 B-419-007-2F

8-Aug-11 B-419-007-5F

8-Aug-11 B-419-007-5F

8-Aug-11 B-419-007-10F

8-Aug-11 B-419-007-10F

8-Aug-11 B-419-007-15F

8-Aug-11 B-419-008-1F

8-Aug-11 B-419-008-1F

8-Aug-11 B-419-008-2F

8-Aug-11 B-419-008-5F

8-Aug-11 B-419-008-5F

8-Aug-11 B-419-008-10F

8-Aug-11 B-419-008-10F

8-Aug-11 B-419-008-15F

3-Aug-11 B-419-010-1F

3-Aug-11 B-419-010-2F

3-Aug-11 B-419-010-5F

3-Aug-11 B-419-010-5F

3-Aug-11 B-419-010-10F

3-Aug-11 B-419-010-10F

3-Aug-11 B-419-010-10F

3-Aug-11 B-419-010-10F

3-Aug-11 B-419-011-1F

3-Aug-11 B-419-011-2F

3-Aug-11 B-419-011-5F

3-Aug-11 B-419-011-5F

3-Aug-11 B-419-011-10F
5 Tritium

10 Mercury

10 Tritium

15 Mercury

15 Tritium

SO 1 Tritium

SO 2 Tritium

SO 5 Mercury

SO 5 Tritium

SO 10 Mercury

SO 10 Tritium

SO 15 Tritium

\section{SO 1 Tritium}

SO 1 Bis(2-

SO 2 Tritium

SO 5 Mercury

SO 5 Tritium

SO 10 Mercury

SO 10 Tritium

SO 15 Tritium

SO 1 Nickel

SO 2 Tritium

SO 5 Mercury

SO 5 Tritium

SO 10 Barium

SO 10 Cadmium

SO 10 Molybdenum

SO 10 Nickel

SO 1 Tritium

SO 2 Tritium

SO 5 Mercury

SO 5 Tritium

SO 10 Mercury

$\begin{array}{cc}1.2 & \mathrm{mg} / \mathrm{kg} \\ 234 & \mathrm{pCi} / \mathrm{g} \\ 0.076 & \mathrm{mg} / \mathrm{kg} \\ 153 & \mathrm{pCi} / \mathrm{g} \\ 0.09 & \mathrm{mg} / \mathrm{kg} \\ 55.2 & \mathrm{pCi} / \mathrm{g}\end{array}$

$163 \mathrm{pCi} / \mathrm{g}$

$307 \mathrm{pCi} / \mathrm{g}$

$0.1 \mathrm{mg} / \mathrm{kg}$

$226 \mathrm{pCi} / \mathrm{g}$

$0.089 \mathrm{mg} / \mathrm{kg}$

$343 \mathrm{pCi} / \mathrm{g}$

$660 \mathrm{pCi} / \mathrm{g}$

\section{$4.05 \quad \mathrm{pCi} / \mathrm{g}$}

$360 \mathrm{ug} / \mathrm{kg}$

$4.49 \mathrm{pCi} / \mathrm{g}$

$0.11 \mathrm{mg} / \mathrm{kg}$

$4.32 \mathrm{pCi} / \mathrm{g}$

$0.092 \mathrm{mg} / \mathrm{kg}$

$1.65 \mathrm{pCi} / \mathrm{g}$

$1.15 \mathrm{pCi} / \mathrm{g}$

$160 \quad \mathrm{mg} / \mathrm{kg} \quad \mathrm{L}$

$0.47 \mathrm{pCi} / \mathrm{g}$

$0.1 \mathrm{mg} / \mathrm{kg}$

$0.372 \mathrm{pCi} / \mathrm{g}$

$940 \mathrm{mg} / \mathrm{kg} \quad \mathrm{L}$

$1.1 \mathrm{mg} / \mathrm{kg}$

$1.7 \mathrm{mg} / \mathrm{kg}$

$140 \mathrm{mg} / \mathrm{kg}$

$1.33 \mathrm{pCi} / \mathrm{g}$

$1.7 \quad \mathrm{pCi} / \mathrm{g}$

$0.076 \mathrm{mg} / \mathrm{kg}$

$1.07 \mathrm{pCi} / \mathrm{g}$

$0.089 \mathrm{mg} / \mathrm{kg}$

1.27

1.27

43

1.27

43

1.27

1.27

1.27

43

1.27

43

1.27

1.27

1.27

120,000

1.27

43

1.27

43

1.27

1.27

20,000

1.27

43

1.27

190,000

800

5,100

20,000

1.27

1.27

43

1.27

43

419 Barkg

$\begin{array}{llll}100 & 1.1 & \mathrm{Y} & \mathrm{N}\end{array}$

0.106

0.0759

0.106

0.0759

0.106

0.106

0.106

0.0759

0.106

0.0759

0.106

0.106

0.106

NE

0.106

0.0759

0.106

0.0759

0.106

0.106

70.1

0.106

0.0759

0.106

332

0.55

1.1

70.1

0.106

0.106

0.0759

0.106

0.0759

$\begin{array}{ll}\mathrm{Y} & \mathrm{N} \\ \mathrm{Y} & \mathrm{Y} \\ \mathrm{Y} & \mathrm{N} \\ \mathrm{Y} & \mathrm{Y} \\ \mathrm{Y} & \mathrm{N} \\ \mathrm{Y} & \mathrm{Y} \\ & \\ \mathrm{Y} & \mathrm{Y} \\ \mathrm{Y} & \mathrm{Y} \\ \mathrm{Y} & \mathrm{N} \\ \mathrm{Y} & \mathrm{Y} \\ \mathrm{Y} & \mathrm{N} \\ \mathrm{Y} & \mathrm{Y} \\ \mathrm{Y} & \mathrm{Y}\end{array}$

Y

$\mathrm{N}$

$\mathrm{Y}$

$\mathrm{Y}$

\section{N}

$$
\text { N }
$$


US EPA

Preliminary Bldg

Industrial Screening

\begin{tabular}{|c|c|c|c|c|c|c|c|c|c|c|c|c|}
\hline Field Location & Sample Date & Sample Identifcation & Matrix & Depth & Analyte & Result & Units & Flag & Level (mg/kg) & Screening Values & established (Y/N) & Above PRG (Y/N) \\
\hline B-419-011 & 3-Aug-11 & B-419-011-DUP-10F & SO & 10 & Mercury & 0.097 & $\mathrm{mg} / \mathrm{kg}$ & & 43 & 0.0759 & $\bar{Y}$ & $\mathrm{~N}$ \\
\hline B-419-011 & 3-Aug-11 & B-419-011-10F & SO & 10 & Tritium & 0.332 & $\mathrm{pCi} / \mathrm{g}$ & & 1.27 & 0.106 & $\mathrm{Y}$ & $\mathrm{N}$ \\
\hline B-419-011 & 3-Aug-11 & B-419-011-15F & SO & 15 & Chromium & 230 & $\mathrm{mg} / \mathrm{kg}$ & $\mathrm{L}$ & $1,500,000(1)$ & 48.9 & $\mathrm{Y}$ & $\mathrm{N}$ \\
\hline B-419-011 & 3-Aug-11 & B-419-011-15F & SO & 15 & Barium & 0.97 & $\mathrm{mg} / \mathrm{L}$ & & NA & NA & NA & NA \\
\hline B-419-011 & 3-Aug-11 & B-419-011-15F & SO & 15 & Barium & 10 & $\mathrm{mg} / \mathrm{L}$ & & NA & NA & NA & NA \\
\hline B-419-011 & 3-Aug-11 & B-419-011-15F & SO & 15 & Cobalt & 0.28 & $\mathrm{mg} / \mathrm{L}$ & & NA & NA & NA & NA \\
\hline B-419-011 & 3-Aug-11 & B-419-011-15F & SO & 15 & Copper & 0.16 & $\mathrm{mg} / \mathrm{L}$ & & NA & NA & NA & NA \\
\hline B-419-011 & 3-Aug-11 & B-419-011-15F & SO & 15 & Lead & 0.088 & $\mathrm{mg} / \mathrm{L}$ & & NA & NA & NA & NA \\
\hline B-419-011 & 3-Aug-11 & B-419-011-15F & SO & 15 & Nickel & 0.45 & $\mathrm{mg} / \mathrm{L}$ & B & NA & NA & NA & NA \\
\hline B-419-011 & 3-Aug-11 & B-419-011-15F & SO & 15 & Vanadium & 0.18 & $\mathrm{mg} / \mathrm{L}$ & & NA & NA & NA & NA \\
\hline B-419-011 & 3-Aug-11 & B-419-011-15F & SO & 15 & Zinc & 2.3 & $\mathrm{mg} / \mathrm{L}$ & & NA & NA & NA & NA \\
\hline B-419-012 & 28-Sep-10 & B-419-012-1F & SO & 1 & Chromium & 54 & $\mathrm{mg} / \mathrm{kg}$ & & $1,500,000(1)$ & 48.9 & $\mathrm{Y}$ & $\mathrm{N}$ \\
\hline B-419-012 & 28-Sep-10 & B-419-012-1F & SO & 1 & Diesel Fuel & 360 & $\mathrm{mg} / \mathrm{kg}$ & $\mathrm{D}$ & 180 & NE & $\mathrm{N}$ & $\mathrm{Y}$ \\
\hline B-419-012 & 28-Sep-10 & B-419-012-1F & SO & 1 & Molybdenum & 4.2 & $\mathrm{mg} / \mathrm{kg}$ & & 5,100 & 1.1 & $\mathrm{Y}$ & $\mathrm{N}$ \\
\hline B-419-012 & 28-Sep-10 & B-419-012-11F & SO & 11 & Mercury & 0.077 & $\mathrm{mg} / \mathrm{kg}$ & & 43 & 0.0759 & $\mathrm{Y}$ & $\mathrm{N}$ \\
\hline B-419-013 & 2-Aug-11 & B-419-013-1F & SO & 1 & Tritium & 0.314 & $\mathrm{pCi} / \mathrm{g}$ & & 1.27 & 0.106 & $\mathrm{Y}$ & $\mathrm{N}$ \\
\hline B-419-013 & 2-Aug-11 & B-419-013-2F & SO & 2 & Tritium & 0.257 & $\mathrm{pCi} / \mathrm{g}$ & & 1.27 & 0.106 & $\mathrm{Y}$ & $\mathrm{N}$ \\
\hline B-419-013 & 2-Aug-11 & B-419-013-5F & SO & 5 & Mercury & 0.14 & $\mathrm{mg} / \mathrm{kg}$ & & 43 & 0.0759 & $\mathrm{Y}$ & $\mathrm{N}$ \\
\hline B-419-013 & 2-Aug-11 & B-419-013-10F & SO & 10 & Mercury & 0.076 & $\mathrm{mg} / \mathrm{kg}$ & & 43 & 0.0759 & $\mathrm{Y}$ & $\mathrm{N}$ \\
\hline B-419-013 & 2-Aug-11 & B-419-013-15F & SO & 15 & Tritium & 0.84 & $\mathrm{pCi} / \mathrm{g}$ & & 1.27 & 0.106 & $\mathrm{Y}$ & $\mathrm{N}$ \\
\hline B-419-014 & 3-Aug-11 & B-419-014-1F & SO & 1 & Hexavalent & 3.5 & $\mathrm{mg} / \mathrm{kg}$ & $\mathrm{D}$ & 5.6 & 2.2 & $\mathrm{Y}$ & $\mathrm{N}$ \\
\hline B-419-014 & 3-Aug-11 & B-419-014-1F & SO & 1 & Tritium & 1.3 & $\mathrm{pCi} / \mathrm{g}$ & & 1.27 & 0.106 & $\mathrm{Y}$ & $\mathrm{Y}$ \\
\hline B-419-014 & 3-Aug-11 & B-419-014-2F & SO & 2 & Lead & 20 & $\mathrm{mg} / \mathrm{kg}$ & $\mathrm{D}$ & 800 & 14.3 & $\mathrm{Y}$ & $\mathrm{N}$ \\
\hline B-419-014 & 3-Aug-11 & B-419-014-2F & SO & 2 & Tritium & 1.05 & $\mathrm{pCi} / \mathrm{g}$ & & 1.27 & 0.106 & $\mathrm{Y}$ & $\mathrm{N}$ \\
\hline B-419-014 & 21-Sep-11 & B-419-014-4.5F & OT & 4.5 & Arsenic & 8.6 & $\mathrm{mg} / \mathrm{kg}$ & & 1.6 & 7.59 & $\mathrm{Y}$ & $\mathrm{Y}$ \\
\hline B-419-014 & 21-Sep-11 & B-419-014-4.5F & OT & 4.5 & Chromium & 760 & $\mathrm{mg} / \mathrm{kg}$ & & $1,500,000(3)$ & 48.9 & $\mathrm{Y}$ & $\mathrm{N}$ \\
\hline B-419-014 & 21-Sep-11 & B-419-014-4.5F & OT & 4.5 & Copper & 180 & $\mathrm{mg} / \mathrm{kg}$ & & 41,000 & 35.8 & $\mathrm{Y}$ & $\mathrm{N}$ \\
\hline B-419-014 & 21-Sep-11 & B-419-014-4.5F & OT & 4.5 & Diesel Fuel & 23 & $\mathrm{mg} / \mathrm{kg}$ & & 180 & NA & $\mathrm{N}$ & $\mathrm{N}$ \\
\hline B-419-014 & 21-Sep-11 & B-419-014-4.5F & OT & 4.5 & Molybdenum & 85 & $\mathrm{mg} / \mathrm{kg}$ & & 5,100 & 1.1 & $\mathrm{Y}$ & $\mathrm{N}$ \\
\hline B-419-014 & 21-Sep-11 & B-419-014-4.5F & OT & 4.5 & Nickel & 440 & $\mathrm{mg} / \mathrm{kg}$ & & 20,000 & 70.1 & $\mathrm{Y}$ & $\mathrm{N}$ \\
\hline
\end{tabular}


US EPA

Preliminary Bldg

Industrial Screening

Field Location

Sample Date Sample Identifcation

Matrix Depth Analyte Result Units Flag

Level (mg/kg)

419 Background background

\begin{tabular}{|c|c|c|}
\hline B-419-014 & 21-Sep-11 & B-419-014-4.5F \\
\hline B-419-014 & 21-Sep-11 & B-419-014-4.5F \\
\hline B-419-014 & 21-Sep-11 & B-419-014-4.5F \\
\hline B-419-014 & 21-Sep-11 & B-419-014-4.5F \\
\hline B-419-014 & 21-Sep-11 & B-419-014-4.5F \\
\hline B-419-014 & 21-Sep-11 & B-419-014-4.5F \\
\hline B-419-014 & 21-Sep-11 & B-419-014-4.5F \\
\hline B-419-014 & 21-Sep-11 & B-419-014-4.5F \\
\hline B-419-014 & 21-Sep-11 & B-419-014-4.5F \\
\hline B-419-014 & 21-Sep-11 & B-419-014-4.5F \\
\hline B-419-014 & 21-Sep-11 & B-419-014-4.5F \\
\hline B-419-014 & 21-Sep-11 & B-419-014-4.5F \\
\hline B-419-014 & 21-Sep-11 & B-419-014-4.5F \\
\hline B-419-014 & 21-Sep-11 & B-419-014-4.5F \\
\hline B-419-014 & 21-Sep-11 & B-419-014-4.5F \\
\hline B-419-014 & 21-Sep-11 & B-419-014-4.5F \\
\hline B-419-014 & 21-Sep-11 & B-419-014-4.5F \\
\hline B-419-014 & 21-Sep-11 & B-419-014-4.5F \\
\hline B-419-014A & 10-Aug-11 & B-419-014A-1F \\
\hline B-419-014A & 10-Aug-11 & B-419-014A-1F \\
\hline B-419-014A & 10-Aug-11 & B-419-014A-1F \\
\hline B-419-014A & 10-Aug-11 & B-419-014A-1F \\
\hline B-419-014A & 10-Aug-11 & B-419-014A-2F \\
\hline B-419-014A & 10-Aug-11 & B-419-014A-DUP-2F \\
\hline B-419-014A & 10-Aug-11 & B-419-014A-2F \\
\hline B-419-014A & 10-Aug-11 & B-419-014A-DUP-2F \\
\hline B-419-014A & 10-Aug-11 & B-419-014A-2F \\
\hline B-419-014A & 10-Aug-11 & B-419-014A-DUP-2F \\
\hline B-419-014A & 10-Aug-11 & B-419-014A-2F \\
\hline B-419-014A & 10-Aug-11 & B-419-014A-2F \\
\hline B-419-014A & 10-Aug-11 & B-419-014A-2F \\
\hline B-419-014A & 10-Aug-11 & B-419-014A-2F \\
\hline B-419-014A & 10-Aug-11 & B-419-014A-2F \\
\hline B-419-014A & 10-Aug-11 & B-419-014A-2F \\
\hline B-419-014A & 10-Aug-11 & B-419-014A-2F \\
\hline B-419-014A & 10-Aug-11 & B-419-014A-2F \\
\hline B-419-014A & 10-Aug-11 & B-419-014A-2F \\
\hline
\end{tabular}

$\begin{array}{lll}\text { OT } & 4.5 & \text { Selenium } \\ \text { OT } & 4.5 & \text { Barium } \\ \text { OT } & 4.5 & \text { Barium } \\ \text { OT } & 4.5 & \text { Chromium } \\ \text { OT } & 4.5 & \text { Cobalt } \\ \text { OT } & 4.5 & \text { Copper } \\ \text { OT } & 4.5 & \text { Lead } \\ \text { OT } & 4.5 & \text { Molybdenum } \\ \text { OT } & 4.5 & \text { Nickel } \\ \text { OT } & 4.5 & \text { Vanadium } \\ \text { OT } & 4.5 & \text { Zinc } \\ \text { OT } & 4.5 & \text { Tritium } \\ \text { OT } & 4.5 & 1,2,4-T r i m e \\ \text { OT } & 4.5 & 1,3,5-T r i m e \\ \text { OT } & 4.5 & \text { Naphthalene } \\ \text { OT } & 4.5 & 1,2,4- \\ \text { OT } & 4.5 & \text { Methylene } \\ \text { OT } & 4.5 & \text { Naphthalene } \\ & & \\ \text { SO } & 1 & \text { Chromium } \\ \text { SO } & 1 & \text { Copper } \\ \text { SO } & 1 & \text { Nickel } \\ \text { SO } & 1 & \text { Tritium } \\ \text { SO } & 2 & \text { Chromium } \\ \text { SO } & 2 & \text { Chromium } \\ \text { SO } & 2 & \text { Cobalt } \\ \text { SO } & 2 & \text { Cobalt } \\ \text { SO } & 2 & \text { Nickel } \\ \text { SO } & 2 & \text { Nickel } \\ \text { SO } & 2 & \text { Barium } \\ \text { SO } & 2 & \text { Barium } \\ \text { SO } & 2 & \text { Chromium } \\ \text { SO } & 2 & \text { Cobalt } \\ \text { SO } & 2 & \text { Copper } \\ \text { SO } & 2 & \text { Lead } \\ \text { SO } & 2 & \text { Nickel } \\ \text { SO } & 2 & \text { Vanadium } \\ \text { SO } & 2 & \text { Zinc }\end{array}$

\begin{tabular}{cccc}
\hline 12 & $\mathrm{mg} / \mathrm{kg}$ & $\mathrm{D}$ \\
\hline 0.59 & $\mathrm{mg} / \mathrm{L}$ & \\
\hline 2.9 & $\mathrm{mg} / \mathrm{L}$ & \\
0.42 & $\mathrm{mg} / \mathrm{L}$ & \\
0.11 & $\mathrm{mg} / \mathrm{L}$ & \\
1.4 & $\mathrm{mg} / \mathrm{L}$ & \\
0.065 & $\mathrm{mg} / \mathrm{L}$ & \\
0.34 & $\mathrm{mg} / \mathrm{L}$ & \\
0.69 & $\mathrm{mg} / \mathrm{L}$ & \\
0.16 & $\mathrm{mg} / \mathrm{L}$ & \\
0.58 & $\mathrm{mg} / \mathrm{L}$ & \\
& 0.382 & $\mathrm{pCi} / \mathrm{g}$ & \\
440 & $\mathrm{ug} / \mathrm{kg}$ & \\
130 & $\mathrm{ug} / \mathrm{kg}$ & \\
140 & $\mathrm{ug} / \mathrm{kg}$ & \\
17 & $\mathrm{ug} / \mathrm{L}$ & $\mathrm{D}$ \\
& 17 & $\mathrm{ug} / \mathrm{L}$ & $\mathrm{BD}$ \\
\hline & 12 & $\mathrm{ug} / \mathrm{L}$ & $\mathrm{D}$
\end{tabular}

5,100
NA
NA
NA
NA
NA
NA
NA
NA
NA
NA
1.27
260,000
$10,000,000$
18,000
NA
NA
NA

Screening Values established (Y/N) Above PRG (Y/N)

B-419-014A

10-Aug-11 B-419-014A-2

$\begin{array}{cc}55 & \mathrm{mg} / \mathrm{kg} \\ 36 & \mathrm{mg} / \mathrm{kg} \\ 160 & \mathrm{mg} / \mathrm{kg} \\ 0.68 & \mathrm{pCi} / \mathrm{g} \\ 64 & \mathrm{mg} / \mathrm{kg} \\ 72 & \mathrm{mg} / \mathrm{kg} \\ 18 & \mathrm{mg} / \mathrm{kg} \\ 19 & \mathrm{mg} / \mathrm{kg} \\ 200 & \mathrm{mg} / \mathrm{kg} \\ 200 & \mathrm{mg} / \mathrm{kg} \\ 0.68 & \mathrm{mg} / \mathrm{L} \\ 6.9 & \mathrm{mg} / \mathrm{L} \\ 0.36 & \mathrm{mg} / \mathrm{L} \\ 0.47 & \mathrm{mg} / \mathrm{L} \\ 0.64 & \mathrm{mg} / \mathrm{L} \\ 0.058 & \mathrm{mg} / \mathrm{L} \\ 2.2 & \mathrm{mg} / \mathrm{L} \\ 0.14 & \mathrm{mg} / \mathrm{L} \\ 0.21 & \mathrm{mg} / \mathrm{L}\end{array}$

$1,500,000(1)$
41,000
20,000
1.27
$1,500,000(1)$
$1,500,000(1)$
300
300
20,000
20,000
NA
NA
NA
NA
NA
NA
NA
NA
NA

\begin{tabular}{|c|c|c|}
\hline 0.55 & $\mathrm{Y}$ & $\mathrm{N}$ \\
\hline NA & NA & NA \\
\hline NA & NA & NA \\
\hline NA & NA & NA \\
\hline NA & NA & NA \\
\hline NA & NA & NA \\
\hline NA & NA & NA \\
\hline NA & NA & NA \\
\hline NA & NA & NA \\
\hline NA & NA & NA \\
\hline NA & NA & NA \\
\hline 0.106 & $\mathrm{Y}$ & $\mathrm{N}$ \\
\hline NA & $\mathrm{N}$ & $\mathrm{N}$ \\
\hline NA & $\mathrm{N}$ & $\mathrm{N}$ \\
\hline NA & $\mathrm{N}$ & $\mathrm{N}$ \\
\hline NA & NA & NA \\
\hline NA & NA & NA \\
\hline NA & NA & NA \\
\hline 48.9 & $\mathrm{Y}$ & $\mathrm{N}$ \\
\hline 35.8 & $\mathrm{Y}$ & $\mathrm{N}$ \\
\hline 70.1 & $\mathrm{Y}$ & $\mathrm{N}$ \\
\hline 0.106 & $\mathrm{Y}$ & $\mathrm{N}$ \\
\hline 48.9 & $\mathrm{Y}$ & $\mathrm{N}$ \\
\hline 48.9 & $\mathrm{Y}$ & $\mathrm{N}$ \\
\hline 16.7 & $\mathrm{Y}$ & $\mathrm{N}$ \\
\hline 16.7 & $\mathrm{Y}$ & $\mathrm{N}$ \\
\hline 70.1 & $\mathrm{Y}$ & $\mathrm{N}$ \\
\hline 70.1 & $\mathrm{Y}$ & $\mathrm{N}$ \\
\hline NA & NA & NA \\
\hline NA & NA & NA \\
\hline NA & NA & NA \\
\hline NA & NA & NA \\
\hline NA & NA & NA \\
\hline NA & NA & NA \\
\hline NA & NA & NA \\
\hline NA & NA & NA \\
\hline NA & NA & NA \\
\hline
\end{tabular}


US EPA

Preliminary Bldg

Industrial Screening

\begin{tabular}{|c|c|c|c|c|c|c|c|c|c|c|c|c|}
\hline Field Location & Sample Date & Sample Identifcation & Matrix & Depth & Analyte & Result & Units & Flag & Level (mg/kg) & Screening Values & established (Y/N) & Above PRG (Y/N) \\
\hline B-419-014A & 10-Aug-11 & B-419-014A-DUP-2F & SO & 2 & Tritium & 0.85 & $\mathrm{pCi} / \mathrm{g}$ & & 1.27 & 0.106 & $\bar{Y}$ & $\mathrm{~N}$ \\
\hline B-419-014A & 10-Aug-11 & B-419-014A-2F & SO & 2 & Tritium & 1.01 & $\mathrm{pCi} / \mathrm{g}$ & & 1.27 & 0.106 & $\mathrm{Y}$ & $\mathrm{N}$ \\
\hline B-419-014A & 10-Aug-11 & B-419-014A-DUP-2F & SO & 2 & $\operatorname{Bis}(2-$ & 5900 & $\mathrm{ug} / \mathrm{kg}$ & & 120,000 & $\mathrm{NE}$ & $\mathrm{N}$ & $\mathrm{N}$ \\
\hline B-419-015A & 15-Aug-11 & B-419-015A-1F & SO & 1 & Barium & 360 & $\mathrm{mg} / \mathrm{kg}$ & $\mathrm{L}$ & 190,000 & 332 & $\mathrm{Y}$ & $\mathrm{N}$ \\
\hline B-419-015A & 15-Aug-11 & B-419-015A-1F & SO & 1 & Zinc & 150 & $\mathrm{mg} / \mathrm{kg}$ & $\mathrm{L}$ & 310,000 & 69.8 & $\mathrm{Y}$ & $\mathrm{N}$ \\
\hline B-419-015A & 15-Aug-11 & B-419-015A-2F & SO & 2 & Barium & 380 & $\mathrm{mg} / \mathrm{kg}$ & $\mathrm{L}$ & 190,000 & 332 & $\mathrm{Y}$ & $\mathrm{N}$ \\
\hline B-419-015A & 15-Aug-11 & B-419-015A-2F & SO & 2 & Zinc & 140 & $\mathrm{mg} / \mathrm{kg}$ & $\mathrm{L}$ & 310,000 & 69.8 & $\mathrm{Y}$ & $\mathrm{N}$ \\
\hline B-419-015A & 15-Aug-11 & B-419-015A-2F & SO & 2 & Tritium & 1.19 & $\mathrm{pCi} / \mathrm{g}$ & $\mathrm{L}$ & 1.27 & 0.106 & $\mathrm{Y}$ & $\mathrm{N}$ \\
\hline B-419-015A & 15-Aug-11 & B-419-015A-5F & SO & 5 & Mercury & 0.077 & $\mathrm{mg} / \mathrm{kg}$ & & 43 & 0.0759 & $\mathrm{Y}$ & $\mathrm{N}$ \\
\hline B-419-015A & 15-Aug-11 & B-419-015A-5F & SO & 5 & Tritium & 0.88 & $\mathrm{pCi} / \mathrm{g}$ & $\mathrm{L}$ & 1.27 & 0.106 & $\mathrm{Y}$ & $\mathrm{N}$ \\
\hline B-419-015A & 15-Aug-11 & B-419-015A-10F & SO & 10 & Arsenic & 37 & $\mathrm{mg} / \mathrm{kg}$ & & 1.6 & 7.59 & $\mathrm{Y}$ & $\mathrm{Y}$ \\
\hline B-419-015A & 15-Aug-11 & B-419-015A-10F & SO & 10 & Barium & 1000 & $\mathrm{mg} / \mathrm{kg}$ & $\mathrm{L}$ & 190,000 & 332 & $\mathrm{Y}$ & $\mathrm{N}$ \\
\hline B-419-015A & 15-Aug-11 & B-419-015A-10F & SO & 10 & Chromium & 230 & $\mathrm{mg} / \mathrm{kg}$ & & $1,500,000(1)$ & 48.9 & $\mathrm{Y}$ & $\mathrm{N}$ \\
\hline B-419-015A & 15-Aug-11 & B-419-015A-10F & SO & 10 & Cobalt & 60 & $\mathrm{mg} / \mathrm{kg}$ & & 300 & 16.7 & $\mathrm{Y}$ & $\mathrm{N}$ \\
\hline B-419-015A & 15-Aug-11 & B-419-015A-10F & SO & 10 & Nickel & 280 & $\mathrm{mg} / \mathrm{kg}$ & & 20,000 & 70.1 & $\mathrm{Y}$ & $\mathrm{N}$ \\
\hline B-419-015A & 15-Aug-11 & B-419-015A-10F & SO & 10 & Vanadium & 180 & $\mathrm{mg} / \mathrm{kg}$ & & 5,200 & 43.7 & $\mathrm{Y}$ & $\mathrm{N}$ \\
\hline B-419-015A & 15-Aug-11 & B-419-015A-10F & SO & 10 & Zinc & 280 & $\mathrm{mg} / \mathrm{kg}$ & $\mathrm{L}$ & 310,000 & 69.8 & $\mathrm{Y}$ & $\mathrm{N}$ \\
\hline B-419-015A & 15-Aug-11 & B-419-015A-15F & SO & 15 & Tritium & 0.426 & $\mathrm{pCi} / \mathrm{g}$ & $\mathrm{L}$ & 1.27 & 0.106 & $\mathrm{Y}$ & $\mathrm{N}$ \\
\hline B-419-016A & 10-Aug-11 & B-419-016A-1F & SO & 1 & Lead & 23 & $\mathrm{mg} / \mathrm{kg}$ & & 800 & 14.3 & $\mathrm{Y}$ & $\mathrm{N}$ \\
\hline B-419-016A & 10-Aug-11 & B-419-016A-1F & SO & 1 & Tritium & 0.444 & $\mathrm{pCi} / \mathrm{g}$ & & 1.27 & 0.106 & $\mathrm{Y}$ & $\mathrm{N}$ \\
\hline B-419-016A & 10-Aug-11 & B-419-016A-2F & SO & 2 & Tritium & 2.39 & $\mathrm{pCi} / \mathrm{g}$ & & 1.27 & 0.106 & $\mathrm{Y}$ & $\mathrm{Y}$ \\
\hline B-419-016A & 10-Aug-11 & B-419-016A-5F & SO & 5 & Tritium & 2.2 & $\mathrm{pCi} / \mathrm{g}$ & & 1.27 & 0.106 & $\mathrm{Y}$ & $\mathrm{Y}$ \\
\hline B-419-016A & 10-Aug-11 & B-419-016A-10F & SO & 10 & Arsenic & 11 & $\mathrm{mg} / \mathrm{kg}$ & & 1.6 & 7.59 & $\mathrm{Y}$ & $\mathrm{Y}$ \\
\hline B-419-016A & 10-Aug-11 & B-419-016A-10F & SO & 10 & Mercury & 0.085 & $\mathrm{mg} / \mathrm{kg}$ & $\mathrm{L}$ & 43 & 0.0759 & $\mathrm{Y}$ & $\mathrm{N}$ \\
\hline B-419-016A & 10-Aug-11 & B-419-016A-10F & SO & 10 & Tritium & 0.336 & $\mathrm{pCi} / \mathrm{g}$ & & 1.27 & 0.106 & $\mathrm{Y}$ & $\mathrm{N}$ \\
\hline B-419-017 & 1-Aug-11 & B-419-017-0.8F & SO & 0.8 & Tritium & 0.55 & $\mathrm{pCi} / \mathrm{g}$ & & 1.27 & 0.106 & $\mathrm{Y}$ & $\mathrm{N}$ \\
\hline B-419-017 & 1-Aug-11 & B-419-017-4.5F & SO & 4.5 & Tritium & 0.64 & $\mathrm{pCi} / \mathrm{g}$ & & 1.27 & 0.106 & $\mathrm{Y}$ & $\mathrm{N}$ \\
\hline B-419-017 & 1-Aug-11 & B-419-017-10F & SO & 10 & Tritium & 0.419 & $\mathrm{pCi} / \mathrm{g}$ & & 1.27 & 0.106 & $\mathrm{Y}$ & $\mathrm{N}$ \\
\hline B-419-017A & 19-Sep-11 & B-419-017A-2F & SO & 2 & Molybdenum & 5.6 & $\mathrm{mg} / \mathrm{kg}$ & & 5,100 & 1.1 & $\mathrm{Y}$ & $\mathrm{N}$ \\
\hline
\end{tabular}


US EPA

Preliminary Bldg

Industrial Screening

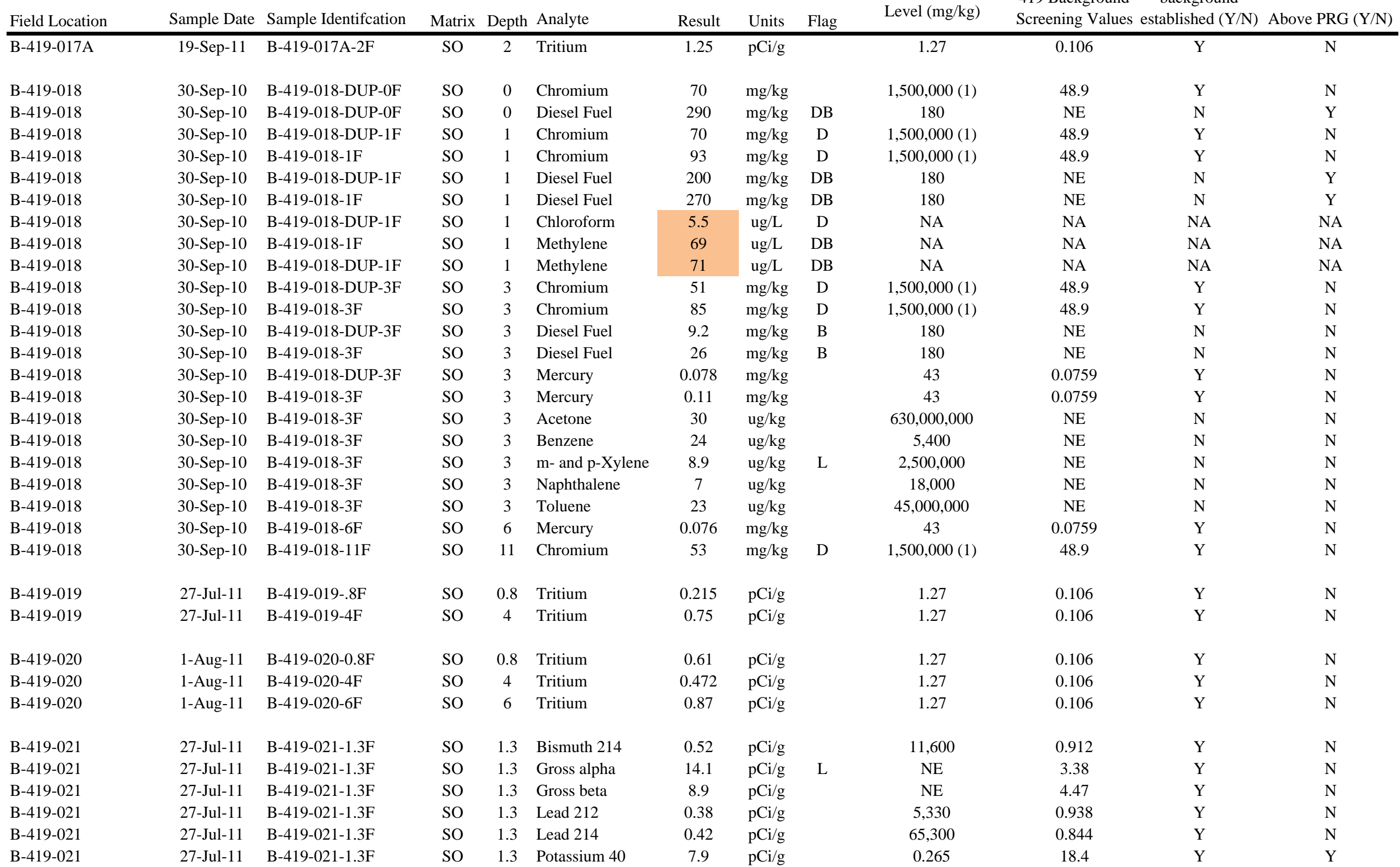


US EPA

Preliminary Bldg

Industrial Screening

\begin{tabular}{|c|c|c|c|c|c|c|c|c|c|c|c|c|}
\hline Field Location & Sample Date & Sample Identifcation & Matrix & Depth & Analyte & Result & Units & Flag & Level (mg/kg) & Screening Values & established (Y/N) & Above PRG (Y/N) \\
\hline B-419-021 & 27-Jul-11 & B-419-021-1.3F & SO & 1.3 & Thorium 228 & 0.52 & $\mathrm{pCi} / \mathrm{g}$ & & 109 & 0.991 & $\mathrm{Y}$ & $\mathrm{N}$ \\
\hline B-419-021 & 27-Jul-11 & B-419-021-1.3F & SO & 1.3 & Thorium 230 & 0.403 & $\mathrm{pCi} / \mathrm{g}$ & & 18 & 0.957 & $\mathrm{Y}$ & $\mathrm{N}$ \\
\hline B-419-021 & 27-Jul-11 & B-419-021-1.3F & SO & 1.3 & Thorium 232 & 0.382 & $\mathrm{pCi} / \mathrm{g}$ & B & 17 & 0.983 & $\mathrm{Y}$ & $\mathrm{N}$ \\
\hline B-419-021 & 27-Jul-11 & B-419-021-1.3F & SO & 1.3 & Tritium & 9.8 & $\mathrm{pCi} / \mathrm{g}$ & & 1.27 & 0.106 & $\mathrm{Y}$ & $\mathrm{Y}$ \\
\hline B-419-021 & 27-Jul-11 & B-419-021-1.3F & SO & 1.3 & Uranium 234 and & 0.42 & $\mathrm{pCi} / \mathrm{g}$ & & 25.5 & 0.76 & $\mathrm{Y}$ & $\mathrm{N}$ \\
\hline B-419-021 & 27-Jul-11 & B-419-021-1.3F & SO & 1.3 & Uranium 238 & 0.33 & $\mathrm{pCi} / \mathrm{g}$ & & 33 & 0.717 & $\mathrm{Y}$ & $\mathrm{N}$ \\
\hline B-419-021 & 27-Jul-11 & B-419-021-4F & SO & 4 & Lead & 96 & $\mathrm{mg} / \mathrm{kg}$ & B & 800 & 14.3 & $\mathrm{Y}$ & $\mathrm{N}$ \\
\hline B-419-021 & 27-Jul-11 & B-419-021-4F & SO & 4 & Mercury & 0.078 & $\mathrm{mg} / \mathrm{kg}$ & & 43 & 0.0759 & $\mathrm{Y}$ & $\mathrm{N}$ \\
\hline B-419-021 & 27-Jul-11 & B-419-021-4F & SO & 4 & Barium & 0.67 & $\mathrm{mg} / \mathrm{L}$ & & NA & NA & NA & NA \\
\hline B-419-021 & 27-Jul-11 & B-419-021-4F & SO & 4 & Barium & 6.5 & $\mathrm{mg} / \mathrm{L}$ & & NA & NA & NA & NA \\
\hline B-419-021 & 27-Jul-11 & B-419-021-4F & SO & 4 & Chromium & 0.15 & $\mathrm{mg} / \mathrm{L}$ & & NA & NA & NA & NA \\
\hline B-419-021 & 27-Jul-11 & B-419-021-4F & SO & 4 & Cobalt & 0.29 & $\mathrm{mg} / \mathrm{L}$ & & NA & NA & NA & NA \\
\hline B-419-021 & 27-Jul-11 & B-419-021-4F & SO & 4 & Copper & 0.12 & $\mathrm{mg} / \mathrm{L}$ & & NA & NA & NA & NA \\
\hline B-419-021 & 27-Jul-11 & B-419-021-4F & SO & 4 & Lead & 0.069 & $\mathrm{mg} / \mathrm{L}$ & B & NA & NA & NA & NA \\
\hline B-419-021 & 27-Jul-11 & B-419-021-4F & SO & 4 & Nickel & 0.59 & $\mathrm{mg} / \mathrm{L}$ & & NA & NA & NA & NA \\
\hline B-419-021 & 27-Jul-11 & B-419-021-4F & SO & 4 & Selenium & 0.055 & $\mathrm{mg} / \mathrm{L}$ & B & NA & NA & NA & NA \\
\hline B-419-021 & 27-Jul-11 & B-419-021-7F & SO & 7 & Mercury & 0.085 & $\mathrm{mg} / \mathrm{kg}$ & & 43 & 0.0759 & $\mathrm{Y}$ & $\mathrm{N}$ \\
\hline B-419-021 & 27-Jul-11 & B-419-021-7F & SO & 7 & Tritium & 1.47 & $\mathrm{pCi} / \mathrm{g}$ & & 1.27 & 0.106 & $\mathrm{Y}$ & $\mathrm{Y}$ \\
\hline B-419-021 & 27-Jul-11 & B-419-021-9F & SO & 9 & Tritium & 0.55 & $\mathrm{pCi} / \mathrm{g}$ & & 1.27 & 0.106 & $\mathrm{Y}$ & $\mathrm{N}$ \\
\hline B-419-021 & 27-Jul-11 & B-419-021-15F & SO & 15 & Diesel Fuel & 11 & $\mathrm{mg} / \mathrm{kg}$ & & 180 & NE & $\mathrm{N}$ & $\mathrm{N}$ \\
\hline B-419-021 & 27-Jul-11 & B-419-021-15F & SO & 15 & Tritium & 0.28 & $\mathrm{pCi} / \mathrm{g}$ & & 1.27 & 0.106 & $\mathrm{Y}$ & $\mathrm{N}$ \\
\hline B-419-022 & 26-Jul-11 & B-419-022-2F & SO & 2 & Selenium & 0.73 & $\mathrm{mg} / \mathrm{kg}$ & & 5,100 & 0.55 & $\mathrm{Y}$ & $\mathrm{N}$ \\
\hline B-419-022 & 26-Jul-11 & B-419-022-2F & SO & 2 & Tritium & 0.6 & $\mathrm{pCi} / \mathrm{g}$ & & 1.27 & 0.106 & $\mathrm{Y}$ & $\mathrm{N}$ \\
\hline B-419-022 & 26-Jul-11 & B-419-022-5F & SO & 5 & Selenium & 0.74 & $\mathrm{mg} / \mathrm{kg}$ & & 5,100 & 0.55 & $\mathrm{Y}$ & $\mathrm{N}$ \\
\hline B-419-022 & 26-Jul-11 & B-419-022-5F & SO & 5 & Tritium & 0.392 & $\mathrm{pCi} / \mathrm{g}$ & & 1.27 & 0.106 & $\mathrm{Y}$ & $\mathrm{N}$ \\
\hline B-419-022 & 26-Jul-11 & B-419-022-10F & SO & 10 & Selenium & 0.6 & $\mathrm{mg} / \mathrm{kg}$ & & 5,100 & 0.55 & $\mathrm{Y}$ & $\mathrm{N}$ \\
\hline B-419-023 & 15-Aug-11 & B-419-023-2F & SO & 2 & Selenium & 0.57 & $\mathrm{mg} / \mathrm{kg}$ & & 5,100 & 0.55 & $\mathrm{Y}$ & $\mathrm{N}$ \\
\hline B-419-023 & 15-Aug-11 & B-419-023-2F & SO & 2 & Tritium & 0.57 & $\mathrm{pCi} / \mathrm{g}$ & $\mathrm{L}$ & 1.27 & 0.106 & $\mathrm{Y}$ & $\mathrm{N}$ \\
\hline B-419-023 & 15-Aug-11 & B-419-023-5F & SO & 5 & Tritium & 1.33 & $\mathrm{pCi} / \mathrm{g}$ & $\mathrm{L}$ & 1.27 & 0.106 & $\mathrm{Y}$ & Y \\
\hline B-419-023 & 15-Aug-11 & B-419-023-15F & SO & 15 & Mercury & 0.12 & mg/kg & & 43 & 0.0759 & Y & $\mathrm{N}$ \\
\hline B-419-024 & 16-Aug-11 & B-419-024-1F & SO & 1 & Diesel Fuel & 64 & $\mathrm{mg} / \mathrm{kg}$ & & 180 & $\mathrm{NE}$ & $\mathrm{N}$ & $\mathrm{N}$ \\
\hline B-419-024 & 16-Aug-11 & B-419-024-1F & SO & 1 & Lead & 16 & $\mathrm{mg} / \mathrm{kg}$ & $\mathrm{D}$ & 800 & 14.3 & $\mathrm{Y}$ & $\mathrm{N}$ \\
\hline
\end{tabular}


US EPA

Preliminary Bldg

Industrial Screening

\begin{tabular}{|c|c|c|c|c|c|c|c|c|c|c|c|c|}
\hline Field Location & Sample Date & Sample Identifcation & Matrix & Depth & Analyte & Result & Units & Flag & Level (mg/kg) & Screening Values & established $(\mathrm{Y} / \mathrm{N})$ & Above PRG $(\mathrm{Y} / \mathrm{N})$ \\
\hline B-419-024 & 16-Aug-11 & B-419-024-1F & SO & 1 & Mercury & 0.28 & $\mathrm{mg} / \mathrm{kg}$ & & 43 & 0.0759 & $\mathrm{Y}$ & $\mathrm{N}$ \\
\hline B-419-024 & 16-Aug-11 & B-419-024-1F & SO & 1 & Americium 241 & 0.68 & $\mathrm{pCi} / \mathrm{g}$ & B & 4.82 & 0.062 & $\mathrm{Y}$ & $\mathrm{N}$ \\
\hline B-419-024 & 16-Aug-11 & B-419-024-1F & SO & 1 & Americium 241 & 0.77 & $\mathrm{pCi} / \mathrm{g}$ & & 4.82 & 0.062 & $\mathrm{Y}$ & $\mathrm{N}$ \\
\hline B-419-024 & 16-Aug-11 & B-419-024-1F & SO & 1 & Gross alpha & 9.8 & $\mathrm{pCi} / \mathrm{g}$ & $\mathrm{L}$ & $\mathrm{NE}$ & 3.38 & $\mathrm{Y}$ & $\mathrm{N}$ \\
\hline B-419-024 & 16-Aug-11 & B-419-024-1F & SO & 1 & Lead 212 & 0.49 & $\mathrm{pCi} / \mathrm{g}$ & & 5,330 & 0.938 & $\mathrm{Y}$ & $\mathrm{N}$ \\
\hline B-419-024 & 16-Aug-11 & B-419-024-1F & SO & 1 & Lead 214 & 0.58 & $\mathrm{pCi} / \mathrm{g}$ & & 65,300 & 0.844 & $\mathrm{Y}$ & $\mathrm{N}$ \\
\hline B-419-024 & 16-Aug-11 & B-419-024-1F & SO & 1 & Plutonium 238 & 0.268 & $\mathrm{pCi} / \mathrm{g}$ & & 14.4 & 0.0355 & $\mathrm{Y}$ & $\mathrm{N}$ \\
\hline B-419-024 & 16-Aug-11 & B-419-024-1F & SO & 1 & Plutonium $239+2 \measuredangle$ & 2.52 & $\mathrm{pCi} / \mathrm{g}$ & & 12.5 & 0.046 & $\mathrm{Y}$ & $\mathrm{N}$ \\
\hline B-419-024 & 16-Aug-11 & B-419-024-1F & SO & 1 & Potassium 40 & 10.3 & $\mathrm{pCi} / \mathrm{g}$ & & 0.265 & 18.4 & $\mathrm{Y}$ & $\mathrm{Y}$ \\
\hline B-419-024 & 16-Aug-11 & B-419-024-1F & SO & 1 & Thallium 208 & 0.33 & $\mathrm{pCi} / \mathrm{g}$ & & 33,600 & 0.371 & $\mathrm{Y}$ & $\mathrm{N}$ \\
\hline B-419-024 & 16-Aug-11 & B-419-024-1F & SO & 1 & Thorium 228 & 0.61 & $\mathrm{pCi} / \mathrm{g}$ & & 109 & 0.991 & $\mathrm{Y}$ & $\mathrm{N}$ \\
\hline B-419-024 & 16-Aug-11 & B-419-024-1F & SO & 1 & Thorium 230 & 0.37 & $\mathrm{pCi} / \mathrm{g}$ & & 18 & 0.957 & $\mathrm{Y}$ & $\mathrm{N}$ \\
\hline B-419-024 & 16-Aug-11 & B-419-024-1F & SO & 1 & Thorium 232 & 0.385 & $\mathrm{pCi} / \mathrm{g}$ & B & 17 & 0.983 & $\mathrm{Y}$ & $\mathrm{N}$ \\
\hline B-419-024 & 16-Aug-11 & B-419-024-1F & SO & 1 & Tritium & 14.2 & $\mathrm{pCi} / \mathrm{g}$ & & 1.27 & 0.106 & $\mathrm{Y}$ & $\mathrm{Y}$ \\
\hline B-419-024 & 16-Aug-11 & B-419-024-1F & SO & 1 & Uranium 234 and & 1.26 & $\mathrm{pCi} / \mathrm{g}$ & & 25.5 & 0.76 & $\mathrm{Y}$ & $\mathrm{N}$ \\
\hline B-419-024 & 16-Aug-11 & B-419-024-1F & SO & 1 & Uranium 238 & 0.89 & $\mathrm{pCi} / \mathrm{g}$ & & 33 & 0.717 & $\mathrm{Y}$ & $\mathrm{N}$ \\
\hline B-419-024 & 16-Aug-11 & B-419-024-2.5F & SO & 2.5 & Diesel Fuel & 86 & $\mathrm{mg} / \mathrm{kg}$ & & 180 & NE & $\mathrm{N}$ & $\mathrm{N}$ \\
\hline B-419-024 & 16-Aug-11 & B-419-024-2.5F & SO & 2.5 & Lead & 61 & $\mathrm{mg} / \mathrm{kg}$ & $\mathrm{D}$ & 800 & 14.3 & $\mathrm{Y}$ & $\mathrm{N}$ \\
\hline B-419-024 & 16-Aug-11 & B-419-024-2.5F & SO & 2.5 & Mercury & 0.6 & $\mathrm{mg} / \mathrm{kg}$ & & 43 & 0.0759 & $\mathrm{Y}$ & $\mathrm{N}$ \\
\hline B-419-024 & 16-Aug-11 & B-419-024-2.5F & SO & 2.5 & Gross alpha & 8.6 & $\mathrm{pCi} / \mathrm{g}$ & $\mathrm{L}$ & NE & 3.38 & $\mathrm{Y}$ & $\mathrm{N}$ \\
\hline B-419-024 & 16-Aug-11 & B-419-024-2.5F & SO & 2.5 & Tritium & 11.6 & $\mathrm{pCi} / \mathrm{g}$ & & 1.27 & 0.106 & $\mathrm{Y}$ & $\mathrm{Y}$ \\
\hline B-419-024 & 16-Aug-11 & B-419-024-2.5F & SO & 2.5 & $\operatorname{Bis}(2-$ & 1200 & $\mathrm{ug} / \mathrm{kg}$ & & 120,000 & NE & $\mathrm{N}$ & $\mathrm{N}$ \\
\hline B-419-024 & 16-Aug-11 & B-419-024-2.5F & SO & 2.5 & РCB 1254 & 6900 & $\mathrm{ug} / \mathrm{kg}$ & DIJ & 740 & NE & $\mathrm{N}$ & $\mathrm{Y}$ \\
\hline B-419-024 & 16-Aug-11 & B-419-024-5F & SO & 5 & Diesel Fuel & 7.5 & mg/kg & & 180 & $\mathrm{NE}$ & $\mathrm{N}$ & $\mathrm{N}$ \\
\hline B-419-024 & 16-Aug-11 & B-419-024-5F & SO & 5 & Mercury & 0.12 & $\mathrm{mg} / \mathrm{kg}$ & & 43 & 0.0759 & $\mathrm{Y}$ & $\mathrm{N}$ \\
\hline B-419-024 & 16-Aug-11 & B-419-024-5F & SO & 5 & Tritium & 5.99 & $\mathrm{pCi} / \mathrm{g}$ & & 1.27 & 0.106 & $\mathrm{Y}$ & $\mathrm{Y}$ \\
\hline B-419-024 & 16-Aug-11 & B-419-024-5F & SO & 5 & $\operatorname{Bis}(2-$ & 460 & $\mathrm{ug} / \mathrm{kg}$ & & 120,000 & NE & $\mathrm{N}$ & $\mathrm{N}$ \\
\hline B-419-024 & 16-Aug-11 & B-419-024-5F & SO & 5 & РCB 1254 & 1000 & $\mathrm{ug} / \mathrm{kg}$ & DIJ & 740 & $\mathrm{NE}$ & $\mathrm{N}$ & $\mathrm{Y}$ \\
\hline B-419-024 & 16-Aug-11 & B-419-024-10F & SO & 10 & Tritium & 0.7 & $\mathrm{pCi} / \mathrm{g}$ & & 1.27 & 0.106 & $\mathrm{Y}$ & $\mathrm{N}$ \\
\hline B-419-024 & 16-Aug-11 & B-419-024-15F & SO & 15 & Tritium & 0.391 & $\mathrm{pCi} / \mathrm{g}$ & & 1.27 & 0.106 & $\mathrm{Y}$ & $\mathrm{N}$ \\
\hline B-419-024A & 22-Aug-11 & B-419-024A-2F & SO & 2 & Tritium & 4.01 & $\mathrm{pCi} / \mathrm{g}$ & & 1.27 & 0.106 & $\mathrm{Y}$ & $\mathrm{Y}$ \\
\hline B-419-025 & 28-Sep-10 & B-419-025-1F & SO & 1 & Diesel Fuel & 130 & $\mathrm{mg} / \mathrm{kg}$ & DBLO & 180 & $\mathrm{NE}$ & $\mathrm{N}$ & $\mathrm{N}$ \\
\hline B-419-025 & 28-Sep-10 & B-419-025-DUP-1F & SO & 1 & Diesel Fuel & 150 & $\mathrm{mg} / \mathrm{kg}$ & DBLO & 180 & $\mathrm{NE}$ & $\mathrm{N}$ & $\mathrm{N}$ \\
\hline
\end{tabular}


US EPA

Preliminary Bldg

Industrial Screening

Level (mg/kg)

background

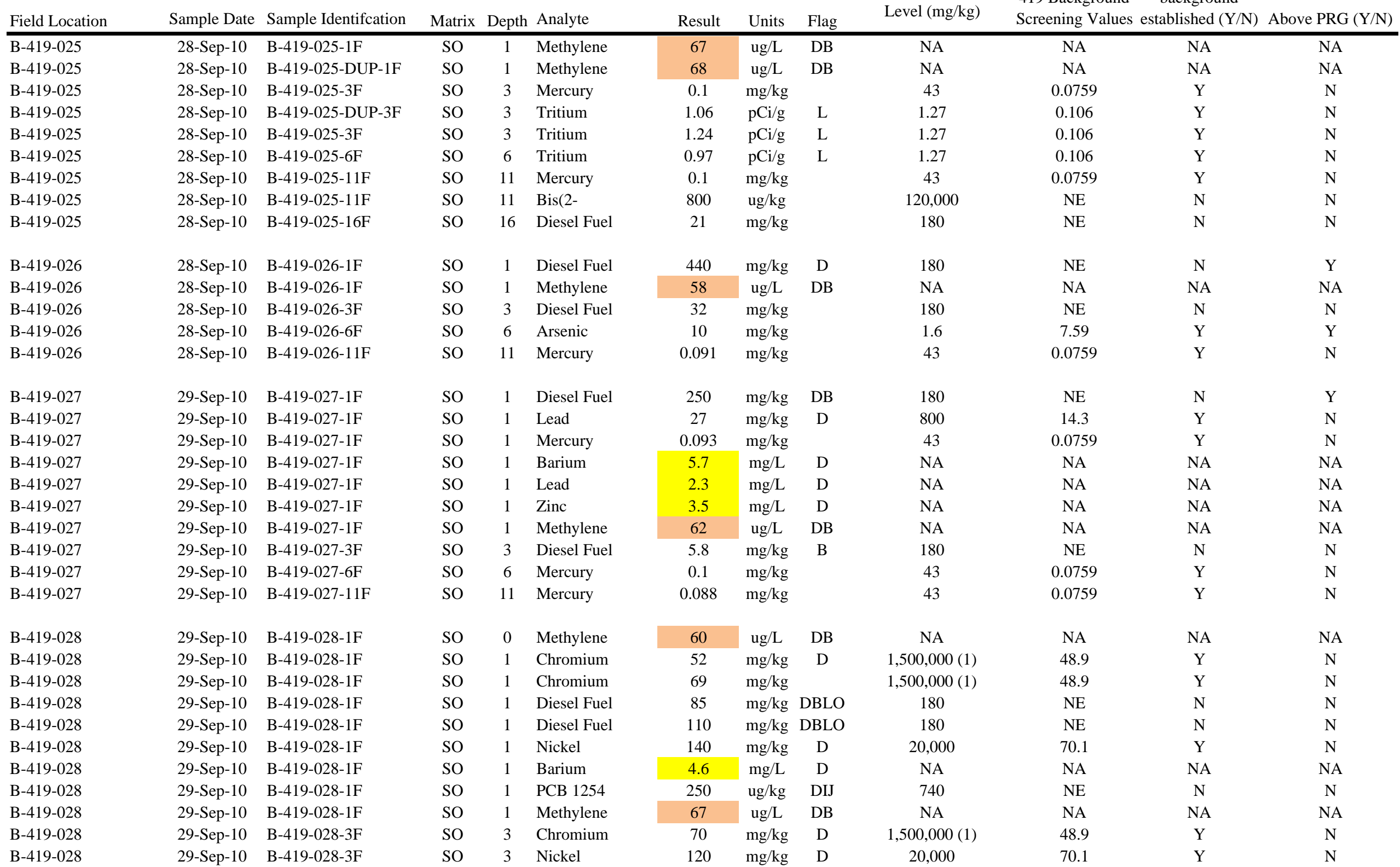


US EPA

Preliminary Bldg

Industrial Screening

\begin{tabular}{|c|c|c|c|c|c|c|c|c|c|c|c|c|}
\hline Field Location & Sample Date & Sample Identifcation & Matrix & Depth & Analyte & Result & Units & Flag & Level (mg/kg) & Screening Values & established (Y/N) & Above PRG (Y/N) \\
\hline B-419-028 & 29-Sep-10 & B-419-028-DUP-3F & SO & 3 & Tritium & 0.283 & $\mathrm{pCi} / \mathrm{g}$ & $\mathrm{L}$ & 1.27 & 0.106 & $\mathrm{Y}$ & $\mathrm{N}$ \\
\hline B-419-028 & 29-Sep-10 & B-419-028-3F & SO & 3 & Tritium & 0.37 & $\mathrm{pCi} / \mathrm{g}$ & $\mathrm{L}$ & 1.27 & 0.106 & $\mathrm{Y}$ & $\mathrm{N}$ \\
\hline B-419-028 & 29-Sep-10 & B-419-028-6F & SO & 6 & Tritium & 4.78 & $\mathrm{pCi} / \mathrm{g}$ & $\mathrm{L}$ & 1.27 & 0.106 & $\mathrm{Y}$ & $\mathrm{Y}$ \\
\hline B-419-028 & 29-Sep-10 & B-419-028-11F & SO & 11 & Tritium & 2.56 & $\mathrm{pCi} / \mathrm{g}$ & $\mathrm{L}$ & 1.27 & 0.106 & $\mathrm{Y}$ & $\mathrm{Y}$ \\
\hline B-419-029 & 29-Sep-10 & B-419-029-1F & SO & 1 & Chromium & 100 & $\mathrm{mg} / \mathrm{kg}$ & $\mathrm{D}$ & $1,500,000(1)$ & 48.9 & $\mathrm{Y}$ & $\mathrm{N}$ \\
\hline B-419-029 & 29-Sep-10 & B-419-029-1F & SO & 1 & Diesel Fuel & 210 & $\mathrm{mg} / \mathrm{kg}$ & DBLO & 180 & $\mathrm{NE}$ & $\mathrm{N}$ & $\mathrm{Y}$ \\
\hline B-419-029 & 29-Sep-10 & B-419-029-1F & SO & 1 & Barium & 9.3 & $\mathrm{mg} / \mathrm{L}$ & $\mathrm{D}$ & NA & NA & NA & NA \\
\hline B-419-029 & 29-Sep-10 & B-419-029-1F & SO & 1 & Lead & 0.54 & $\mathrm{mg} / \mathrm{L}$ & $\mathrm{D}$ & NA & NA & NA & NA \\
\hline B-419-029 & 29-Sep-10 & B-419-029-1F & SO & 1 & Methylene & 69 & ug/L & DB & NA & NA & NA & NA \\
\hline B-419-029 & 29-Sep-10 & B-419-029-6F & SO & 6 & Diesel Fuel & 360 & $\mathrm{mg} / \mathrm{kg}$ & DBLO & 180 & NE & $\mathrm{N}$ & $\mathrm{Y}$ \\
\hline B-419-029 & 29-Sep-10 & B-419-029-11F & SO & 11 & Mercury & 0.082 & $\mathrm{mg} / \mathrm{kg}$ & & 43 & 0.0759 & $\mathrm{Y}$ & $\mathrm{N}$ \\
\hline B-419-030 & 30-Sep-10 & B-419-030-1F & SO & 1 & Chromium & 110 & $\mathrm{mg} / \mathrm{kg}$ & $\mathrm{D}$ & $1,500,000(1)$ & 48.9 & $\mathrm{Y}$ & $\mathrm{N}$ \\
\hline B-419-030 & 30-Sep-10 & B-419-030-1F & so & 1 & Diesel Fuel & 140 & $\mathrm{mg} / \mathrm{kg}$ & DB & 180 & NE & $\mathrm{N}$ & $\mathrm{N}$ \\
\hline B-419-030 & 30-Sep-10 & B-419-030-3F & SO & 3 & Chromium & 71 & $\mathrm{mg} / \mathrm{kg}$ & $\mathrm{D}$ & $1,500,000(1)$ & 48.9 & $\mathrm{Y}$ & $\mathrm{N}$ \\
\hline B-419-030 & 30-Sep-10 & B-419-030-3F & SO & 3 & Diesel Fuel & 20 & $\mathrm{mg} / \mathrm{kg}$ & B & 180 & $\mathrm{NE}$ & $\mathrm{N}$ & $\mathrm{N}$ \\
\hline B-419-030 & 30-Sep-10 & B-419-030-3F & SO & 3 & Mercury & 0.21 & $\mathrm{mg} / \mathrm{kg}$ & & 43 & 0.0759 & $\mathrm{Y}$ & $\mathrm{N}$ \\
\hline B-419-030 & 30-Sep-10 & B-419-030-6F & SO & 6 & Arsenic & 9.2 & $\mathrm{mg} / \mathrm{kg}$ & $\mathrm{D}$ & 1.6 & 7.59 & $\mathrm{Y}$ & $\mathrm{Y}$ \\
\hline B-419-030 & 30-Sep-10 & B-419-030-6F & so & 6 & Chromium & 59 & $\mathrm{mg} / \mathrm{kg}$ & $\mathrm{D}$ & $1,500,000(1)$ & 48.9 & $\mathrm{Y}$ & $\mathrm{N}$ \\
\hline B-419-030 & 30-Sep-10 & B-419-030-6F & SO & 6 & Mercury & 0.089 & $\mathrm{mg} / \mathrm{kg}$ & & 43 & 0.0759 & $\mathrm{Y}$ & $\mathrm{N}$ \\
\hline B-419-030 & 30-Sep-10 & B-419-030-11F & SO & 11 & Mercury & 0.086 & $\mathrm{mg} / \mathrm{kg}$ & & 43 & 0.0759 & $\mathrm{Y}$ & $\mathrm{N}$ \\
\hline B-419-030 & 30-Sep-10 & B-419-030-11F & SO & 11 & Tritium & 0.77 & $\mathrm{pCi} / \mathrm{g}$ & & 1.27 & 0.106 & $\mathrm{Y}$ & $\mathrm{N}$ \\
\hline B-419-031 & 17-Aug-11 & B-419-031-1F & SO & 1 & Zinc & 76 & $\mathrm{mg} / \mathrm{kg}$ & $\mathrm{L}$ & 310,000 & 69.8 & $\mathrm{Y}$ & $\mathrm{N}$ \\
\hline B-419-031 & 17-Aug-11 & B-419-031-2F & SO & 2 & Diesel Fuel & 5.8 & mg/kg & & 180 & $\mathrm{NE}$ & $\mathrm{N}$ & $\mathrm{N}$ \\
\hline B-419-031 & 17-Aug-11 & B-419-031-5F & SO & 5 & Mercury & 0.099 & $\mathrm{mg} / \mathrm{kg}$ & & 43 & 0.0759 & $\mathrm{Y}$ & $\mathrm{N}$ \\
\hline B-419-031 & 17-Aug-11 & B-419-031-15F & SO & 15 & Mercury & 0.11 & $\mathrm{mg} / \mathrm{kg}$ & & 43 & 0.0759 & Y & $\mathrm{N}$ \\
\hline B-419-032 & 1-Aug-11 & B-419-032-0.8F & SO & 0.8 & Diesel Fuel & 15 & $\mathrm{mg} / \mathrm{kg}$ & & 180 & $\mathrm{NE}$ & $\mathrm{N}$ & $\mathrm{N}$ \\
\hline B-419-032 & 1-Aug-11 & B-419-032-0.8F & SO & 0.8 & Mercury & 0.083 & $\mathrm{mg} / \mathrm{kg}$ & & 43 & 0.0759 & Y & $\mathrm{N}$ \\
\hline B-419-032 & 1-Aug-11 & B-419-032-5F & SO & 5 & Tritium & 0.92 & $\mathrm{pCi} / \mathrm{g}$ & & 1.27 & 0.106 & $\mathrm{Y}$ & $\mathrm{N}$ \\
\hline B-419-032 & 1-Aug-11 & B-419-032-10F & SO & 10 & Nickel & 100 & $\mathrm{mg} / \mathrm{kg}$ & & 20,000 & 70.1 & $\mathrm{Y}$ & $\mathrm{N}$ \\
\hline
\end{tabular}


US EPA

Preliminary Bldg

Industrial Screening

background

\begin{tabular}{|c|c|c|c|c|c|c|c|c|c|c|c|c|}
\hline Field Location & Sample Date & Sample Identifcation & Matrix & Depth & Analyte & Result & Units & Flag & Level (mg/kg) & Screening Values & established (Y/N) & Above PRG (Y/N) \\
\hline B-419-032 & 1-Aug-11 & B-419-032-15F & SO & 15 & Tritium & 0.225 & $\mathrm{pCi} / \mathrm{g}$ & & 1.27 & 0.106 & $\mathrm{Y}$ & $\mathrm{N}$ \\
\hline B-419-032A & 19-Sep-11 & B-419-032A-2F & SO & 2 & Tritium & 0.37 & $\mathrm{pCi} / \mathrm{g}$ & & 1.27 & 0.106 & $\mathrm{Y}$ & $\mathrm{N}$ \\
\hline B-419-033 & 16-Aug-11 & B-419-033-1.6F & SO & 1.6 & Cobalt & 19 & $\mathrm{mg} / \mathrm{kg}$ & LO & 300 & 16.7 & $\mathrm{Y}$ & $\mathrm{N}$ \\
\hline B-419-033 & 16-Aug-11 & B-419-033-1.6F & SO & 1.6 & Tritium & 0.338 & $\mathrm{pCi} / \mathrm{g}$ & & 1.27 & 0.106 & $\mathrm{Y}$ & $\mathrm{N}$ \\
\hline B-419-033 & 16-Aug-11 & B-419-033-2F & SO & 2 & Cobalt & 21 & $\mathrm{mg} / \mathrm{kg}$ & LO & 300 & 16.7 & $\mathrm{Y}$ & $\mathrm{N}$ \\
\hline B-419-033 & 16-Aug-11 & B-419-033-2F & SO & 2 & Tritium & 0.313 & $\mathrm{pCi} / \mathrm{g}$ & & 1.27 & 0.106 & $\mathrm{Y}$ & $\mathrm{N}$ \\
\hline B-419-034 & 17-Aug-11 & B-419-034-1F & SO & 1 & Diesel Fuel & 23 & $\mathrm{mg} / \mathrm{kg}$ & & 180 & NE & $\mathrm{N}$ & $\mathrm{N}$ \\
\hline B-419-034 & 17-Aug-11 & B-419-034-1F & SO & 1 & Tetrachloroethen & 5.6 & ug/kg & & 2,600 & NE & $\mathrm{N}$ & $\mathrm{N}$ \\
\hline B-419-034 & 17-Aug-11 & B-419-034-2F & SO & 2 & Chromium & 54 & $\mathrm{mg} / \mathrm{kg}$ & & 1,500,000 (1) & 48.9 & $\mathrm{Y}$ & $\mathrm{N}$ \\
\hline B-419-034 & 17-Aug-11 & B-419-034-2F & SO & 2 & Diesel Fuel & 54 & $\mathrm{mg} / \mathrm{kg}$ & & 180 & $\mathrm{NE}$ & $\mathrm{N}$ & $\mathrm{N}$ \\
\hline B-419-034 & 17-Aug-11 & B-419-034-2F & SO & 2 & Nickel & 110 & $\mathrm{mg} / \mathrm{kg}$ & & 20,000 & 70.1 & $\mathrm{Y}$ & $\mathrm{N}$ \\
\hline B-419-034 & 17-Aug-11 & B-419-034-2F & SO & 2 & Tritium & 0.278 & $\mathrm{pCi} / \mathrm{g}$ & & 1.27 & 0.106 & $\mathrm{Y}$ & $\mathrm{N}$ \\
\hline B-419-034 & 17-Aug-11 & B-419-034-2F & SO & 2 & Tetrachloroethen & 5.4 & ug/kg & & 2,600 & $\mathrm{NE}$ & $\mathrm{N}$ & $\mathrm{N}$ \\
\hline B-419-034 & 17-Aug-11 & B-419-034-5F & SO & 5 & Mercury & 0.099 & $\mathrm{mg} / \mathrm{kg}$ & & 43 & 0.0759 & $\mathrm{Y}$ & $\mathrm{N}$ \\
\hline B-419-034 & 17-Aug-11 & B-419-034-5F & SO & 5 & Tritium & 1.44 & $\mathrm{pCi} / \mathrm{g}$ & & 1.27 & 0.106 & $\mathrm{Y}$ & $\mathrm{Y}$ \\
\hline B-419-034 & 17-Aug-11 & B-419-034-10F & SO & 10 & Tritium & 1.8 & $\mathrm{pCi} / \mathrm{g}$ & & 1.27 & 0.106 & $\mathrm{Y}$ & $\mathrm{Y}$ \\
\hline B-419-034 & 17-Aug-11 & B-419-034-15F & SO & 15 & Tritium & 1.87 & $\mathrm{pCi} / \mathrm{g}$ & & 1.27 & 0.106 & $\mathrm{Y}$ & $\mathrm{Y}$ \\
\hline B-419-035 & 27-Sep-10 & B-419-035-1F & SO & 1 & Cadmium & 0.73 & $\mathrm{mg} / \mathrm{kg}$ & & 800 & 0.55 & $\mathrm{Y}$ & $\mathrm{N}$ \\
\hline B-419-035 & 27-Sep-10 & B-419-035-1F & SO & 1 & Chromium & 54 & $\mathrm{mg} / \mathrm{kg}$ & $\mathrm{L}$ & $1,500,000(1)$ & 48.9 & $\mathrm{Y}$ & $\mathrm{N}$ \\
\hline B-419-035 & 27-Sep-10 & B-419-035-1F & SO & 1 & Diesel Fuel & 480 & $\mathrm{mg} / \mathrm{kg}$ & $\mathrm{D}$ & 180 & NE & $\mathrm{N}$ & $\mathrm{Y}$ \\
\hline B-419-035 & 27-Sep-10 & B-419-035-1F & SO & 1 & Mercury & 0.19 & $\mathrm{mg} / \mathrm{kg}$ & B & 43 & 0.0759 & $\mathrm{Y}$ & $\mathrm{N}$ \\
\hline B-419-035 & 27-Sep-10 & B-419-035-1F & SO & 1 & Nickel & 140 & $\mathrm{mg} / \mathrm{kg}$ & LO & 20,000 & 70.1 & $\mathrm{Y}$ & $\mathrm{N}$ \\
\hline B-419-035 & 27-Sep-10 & B-419-039-3F & SO & 1 & Barium & 11 & $\mathrm{mg} / \mathrm{L}$ & $\mathrm{D}$ & NA & NA & NA & NA \\
\hline B-419-035 & 27-Sep-10 & B-419-035-1F & SO & 1 & Tritium & 0.213 & $\mathrm{pCi} / \mathrm{g}$ & $\mathrm{L}$ & 1.27 & 0.106 & $\mathrm{Y}$ & $\mathrm{N}$ \\
\hline B-419-035 & 27-Sep-10 & B-419-035-1F & SO & 1 & Methylene & 50 & ug/L & $\mathrm{D}$ & NA & NA & NA & NA \\
\hline B-419-035 & 27-Sep-10 & B-419-035-3F & SO & 3 & Chromium & 52 & $\mathrm{mg} / \mathrm{kg}$ & $\mathrm{L}$ & 1,500,000 (1) & 48.9 & $\mathrm{Y}$ & $\mathrm{N}$ \\
\hline B-419-035 & 27-Sep-10 & B-419-035-3F & SO & 3 & Diesel Fuel & 370 & $\mathrm{mg} / \mathrm{kg}$ & $\mathrm{D}$ & 180 & NE & $\mathrm{N}$ & $\mathrm{Y}$ \\
\hline B-419-035 & 27-Sep-10 & B-419-035-3F & SO & 3 & Nickel & 110 & $\mathrm{mg} / \mathrm{kg}$ & LO & 20,000 & 70.1 & $\mathrm{Y}$ & $\mathrm{N}$ \\
\hline B-419-035 & 27-Sep-10 & B-419-035-11F & SO & 11 & Mercury & 0.11 & $\mathrm{mg} / \mathrm{kg}$ & B & 43 & 0.0759 & $\mathrm{Y}$ & $\mathrm{N}$ \\
\hline
\end{tabular}


US EPA

Preliminary Bldg

Industrial Screening

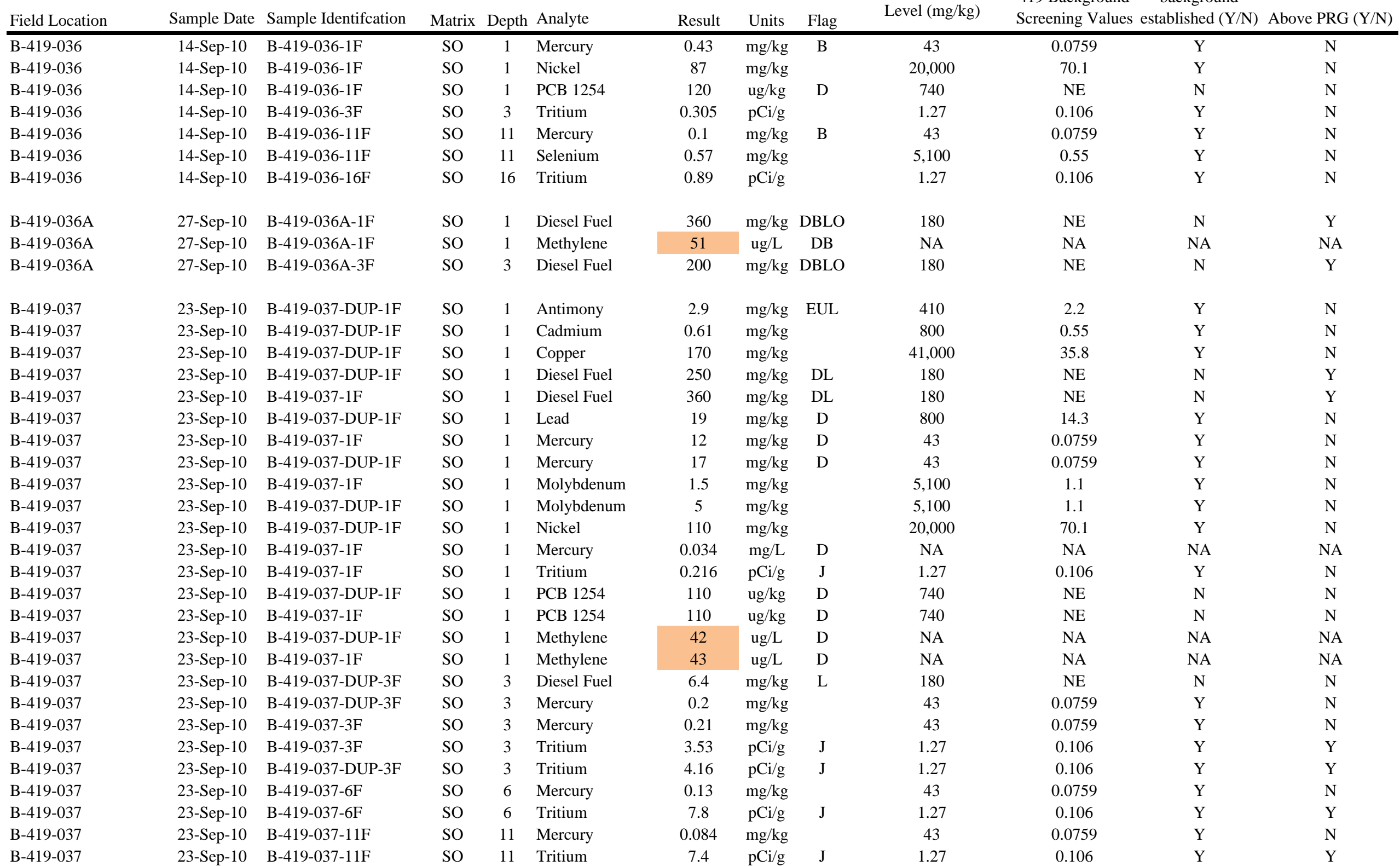


US EPA

Preliminary Bldg

Industrial Screening 419 Backround

background

Field Location $\quad$ Sample Date Sample Identifcation Matrix Depth Analyte Result Units Flag Screening Values established (Y/N) Above PRG (Y/N)

\begin{tabular}{|c|c|c|}
\hline B-419-038 & 24-Aug-11 & B-419-038-1F \\
\hline B-419-038 & 24-Aug-11 & B-419-038-1F \\
\hline B-419-038 & 24-Aug-11 & B-419-038-1F \\
\hline B-419-038 & 24-Aug-11 & B-419-038-1F \\
\hline B-419-038 & 24-Aug-11 & B-419-038-1F \\
\hline B-419-038 & 24-Aug-11 & B-419-038-1F \\
\hline B-419-038 & 24-Aug-11 & B-419-038-1F \\
\hline B-419-038 & 24-Aug-11 & B-419-038-1F \\
\hline B-419-038 & 24-Aug-11 & B-419-038-1F \\
\hline B-419-038 & 24-Aug-11 & B-419-038-1F \\
\hline B-419-038 & 24-Aug-11 & B-419-038-1F \\
\hline B-419-038 & 24-Aug-11 & B-419-038-1F \\
\hline B-419-038 & 24-Aug-11 & B-419-038-1F \\
\hline B-419-038 & 24-Aug-11 & B-419-038-1F \\
\hline B-419-038 & 24-Aug-11 & B-419-038-1F \\
\hline B-419-038 & 24-Aug-11 & B-419-038-1F \\
\hline B-419-038 & 24-Aug-11 & B-419-038-2F \\
\hline B-419-038 & 24-Aug-11 & B-419-038-2F \\
\hline B-419-038 & 24-Aug-11 & B-419-038-2F \\
\hline B-419-038 & 24-Aug-11 & B-419-038-2F \\
\hline B-419-038 & 24-Aug-11 & B-419-038-2F \\
\hline B-419-038 & 24-Aug-11 & B-419-038-2F \\
\hline B-419-038 & 24-Aug-11 & B-419-038-2F \\
\hline B-419-038 & 24-Aug-11 & B-419-038-2F \\
\hline B-419-038 & 24-Aug-11 & B-419-038-2F \\
\hline B-419-038 & 24-Aug-11 & B-419-038-2F \\
\hline B-419-038 & 24-Aug-11 & B-419-038-2F \\
\hline B-419-038 & 24-Aug-11 & B-419-038-2F \\
\hline B-419-038 & 24-Aug-11 & B-419-038-2F \\
\hline B-419-038 & 24-Aug-11 & B-419-038-2F \\
\hline B-419-038 & 24-Aug-11 & B-419-038-2F \\
\hline B-419-038 & 24-Aug-11 & B-419-038-2F \\
\hline B-419-038 & 24-Aug-11 & B-419-038-2F \\
\hline B-419-038 & 24-Aug-11 & B-419-038-2F \\
\hline B-419-038 & 24-Aug-11 & B-419-038-2F \\
\hline B-419-038 & 24-Aug-11 & B-419-038-2F \\
\hline В-419-038 & 24-Aug-11 & B-419-038-2F \\
\hline
\end{tabular}

$\begin{array}{lll}\text { SO } & 1 & \text { Beryllium } \\ \text { SO } & 1 & \text { Lead } \\ \text { SO } & 1 & \text { Mercury } \\ \text { SO } & 1 & \text { Barium } \\ \text { SO } & 1 & \text { Barium } \\ \text { SO } & 1 & \text { Chromium } \\ \text { SO } & 1 & \text { Cobalt } \\ \text { SO } & 1 & \text { Copper } \\ \text { SO } & 1 & \text { Lead } \\ \text { SO } & 1 & \text { Lead } \\ \text { SO } & 1 & \text { Nickel } \\ \text { SO } & 1 & \text { Vanadium } \\ \text { SO } & 1 & \text { Zinc } \\ \text { SO } & 1 & \text { Gross alpha } \\ \text { SO } & 1 & \text { Tritium } \\ \text { SO } & 1 & \text { PCB 1248 } \\ \text { SO } & 2 & \text { Beryllium } \\ \text { SO } & 2 & \text { Cadmium } \\ \text { SO } & 2 & \text { Chromium } \\ \text { SO } & 2 & \text { Copper } \\ \text { SO } & 2 & \text { Diesel Fuel } \\ \text { SO } & 2 & \text { Hexavalent } \\ \text { SO } & 2 & \text { Lead } \\ \text { SO } & 2 & \text { Mercury } \\ \text { SO } & 2 & \text { Silver } \\ \text { SO } & 2 & \text { Zinc } \\ \text { SO } & 2 & \text { Barium } \\ \text { SO } & 2 & \text { Barium } \\ \text { SO } & 2 & \text { Beryllium } \\ \text { SO } & 2 & \text { Beryllium } \\ \text { SO } & 2 & \text { Cadmium } \\ \text { SO } & 2 & \text { Chromium } \\ \text { SO } & 2 & \text { Chromium } \\ \text { SO } & 2 & \text { Cobalt } \\ \text { SO } & 2 & \text { Copper } \\ \text { SO } & 2 & \text { Lead } \\ \text { SO } & 2 & \text { Lead }\end{array}$

\begin{tabular}{|c|c|c|c|}
\hline 0.69 & $\mathrm{mg} / \mathrm{kg}$ & & 2,000 \\
\hline 220 & $\mathrm{mg} / \mathrm{kg}$ & $\mathrm{O}$ & 800 \\
\hline 0.65 & $\mathrm{mg} / \mathrm{kg}$ & $\mathrm{O}$ & 43 \\
\hline 0.81 & $\mathrm{mg} / \mathrm{L}$ & & NA \\
\hline 11 & $\mathrm{mg} / \mathrm{L}$ & & NA \\
\hline 0.38 & $\mathrm{mg} / \mathrm{L}$ & & NA \\
\hline 0.4 & $\mathrm{mg} / \mathrm{L}$ & & NA \\
\hline 0.23 & $\mathrm{mg} / \mathrm{L}$ & & NA \\
\hline 0.28 & $\mathrm{mg} / \mathrm{L}$ & & NA \\
\hline 1.5 & $\mathrm{mg} / \mathrm{L}$ & & NA \\
\hline 0.41 & $\mathrm{mg} / \mathrm{L}$ & B & NA \\
\hline 0.47 & $\mathrm{mg} / \mathrm{L}$ & & NA \\
\hline 1.7 & $\mathrm{mg} / \mathrm{L}$ & & NA \\
\hline 5.6 & $\mathrm{pCi} / \mathrm{g}$ & & $\mathrm{NE}$ \\
\hline 390 & $\mathrm{pCi} / \mathrm{g}$ & $\mathrm{J}$ & 1.27 \\
\hline 130 & $\mathrm{ug} / \mathrm{kg}$ & & 740 \\
\hline 29 & $\mathrm{mg} / \mathrm{kg}$ & & 2,000 \\
\hline 2.1 & $\mathrm{mg} / \mathrm{kg}$ & & 800 \\
\hline 280 & $\mathrm{mg} / \mathrm{kg}$ & $\mathrm{L}$ & $1,500,000(1)$ \\
\hline 110 & $\mathrm{mg} / \mathrm{kg}$ & & 41,000 \\
\hline 80 & $\mathrm{mg} / \mathrm{kg}$ & & 180 \\
\hline 8.3 & $\mathrm{mg} / \mathrm{kg}$ & D & 5.6 \\
\hline 230 & $\mathrm{mg} / \mathrm{kg}$ & $\mathrm{O}$ & 800 \\
\hline 66 & $\mathrm{mg} / \mathrm{kg}$ & DO & 43 \\
\hline 1.8 & $\mathrm{mg} / \mathrm{kg}$ & & 5,100 \\
\hline 130 & $\mathrm{mg} / \mathrm{kg}$ & & 310,000 \\
\hline 0.91 & $\mathrm{mg} / \mathrm{L}$ & & NA \\
\hline 9.4 & $\mathrm{mg} / \mathrm{L}$ & & NA \\
\hline 0.06 & $\mathrm{mg} / \mathrm{L}$ & B & NA \\
\hline 1.1 & $\mathrm{mg} / \mathrm{L}$ & & NA \\
\hline 0.093 & $\mathrm{mg} / \mathrm{L}$ & & NA \\
\hline 0.15 & $\mathrm{mg} / \mathrm{L}$ & & NA \\
\hline 7.5 & $\mathrm{mg} / \mathrm{L}$ & & NA \\
\hline 0.26 & $\mathrm{mg} / \mathrm{L}$ & & NA \\
\hline 3.9 & $\mathrm{mg} / \mathrm{L}$ & & NA \\
\hline 0.16 & $\mathrm{mg} / \mathrm{L}$ & & NA \\
\hline 8.3 & $\mathrm{mg} / \mathrm{L}$ & & NA \\
\hline
\end{tabular}

\begin{tabular}{|c|c|c|}
\hline 0.55 & $\mathrm{Y}$ & $\mathrm{N}$ \\
\hline 14.3 & $\mathrm{Y}$ & $\mathrm{N}$ \\
\hline 0.0759 & $\mathrm{Y}$ & $\mathrm{N}$ \\
\hline NA & NA & NA \\
\hline NA & NA & NA \\
\hline NA & NA & NA \\
\hline NA & NA & NA \\
\hline NA & NA & NA \\
\hline NA & NA & NA \\
\hline NA & NA & NA \\
\hline NA & NA & NA \\
\hline NA & NA & NA \\
\hline NA & NA & NA \\
\hline 3.38 & $\mathrm{Y}$ & $\mathrm{N}$ \\
\hline 0.106 & $\mathrm{Y}$ & $\mathrm{Y}$ \\
\hline NE & $\mathrm{N}$ & $\mathrm{N}$ \\
\hline 0.55 & $\mathrm{Y}$ & $\mathrm{N}$ \\
\hline 0.55 & $\mathrm{Y}$ & $\mathrm{N}$ \\
\hline 48.9 & $\mathrm{Y}$ & $\mathrm{N}$ \\
\hline 35.8 & $\mathrm{Y}$ & $\mathrm{N}$ \\
\hline NE & $\mathrm{N}$ & $\mathrm{N}$ \\
\hline 2.2 & Y & $\mathrm{Y}$ \\
\hline 14.3 & $\mathrm{Y}$ & $\mathrm{N}$ \\
\hline 0.0759 & $\mathrm{Y}$ & $\mathrm{Y}$ \\
\hline 1.1 & $\mathrm{Y}$ & $\mathrm{N}$ \\
\hline 69.8 & $\mathrm{Y}$ & $\mathrm{N}$ \\
\hline NA & NA & NA \\
\hline NA & NA & NA \\
\hline NA & NA & NA \\
\hline NA & NA & NA \\
\hline NA & NA & NA \\
\hline NA & NA & NA \\
\hline NA & NA & NA \\
\hline NA & NA & NA \\
\hline NA & NA & NA \\
\hline NA & NA & NA \\
\hline NA & NA & NA \\
\hline
\end{tabular}


US EPA

Preliminary Bldg

Industrial Screening

background

\begin{tabular}{|c|c|c|c|c|c|c|c|c|c|c|c|c|}
\hline Field Location & Sample Date & Sample Identifcation & Matrix & Depth & Analyte & Result & Units & Flag & Level (mg/kg) & Screening Values & established (Y/N) & Above PRG (Y/N) \\
\hline B-419-038 & 24-Aug-11 & B-419-038-2F & SO & 2 & Mercury & 0.073 & $\mathrm{mg} / \mathrm{L}$ & & NA & NA & NA & NA \\
\hline B-419-038 & 24-Aug-11 & B-419-038-2F & SO & 2 & Mercury & 0.56 & $\mathrm{mg} / \mathrm{L}$ & $\mathrm{D}$ & NA & NA & NA & NA \\
\hline B-419-038 & 24-Aug-11 & B-419-038-2F & SO & 2 & Nickel & 1.9 & $\mathrm{mg} / \mathrm{L}$ & B & NA & NA & NA & NA \\
\hline B-419-038 & 24-Aug-11 & B-419-038-2F & SO & 2 & Zinc & 0.25 & $\mathrm{mg} / \mathrm{L}$ & & NA & NA & NA & NA \\
\hline B-419-038 & 24-Aug-11 & B-419-038-2F & SO & 2 & Zinc & 6.1 & $\mathrm{mg} / \mathrm{L}$ & & NA & NA & NA & NA \\
\hline B-419-038 & 24-Aug-11 & B-419-038-2F & SO & 2 & Actinium 228 & 0.97 & $\mathrm{pCi} / \mathrm{g}$ & & 988 & 1.5 & $\mathrm{Y}$ & $\mathrm{N}$ \\
\hline B-419-038 & 24-Aug-11 & B-419-038-2F & SO & 2 & Americium 241 & 132 & $\mathrm{pCi} / \mathrm{g}$ & B & 4.82 & 0.062 & $\mathrm{Y}$ & $\mathrm{Y}$ \\
\hline B-419-038 & 24-Aug-11 & B-419-038-2F & SO & 2 & Americium 241 & 150 & $\mathrm{pCi} / \mathrm{g}$ & & 4.82 & 0.062 & $\mathrm{Y}$ & $\mathrm{Y}$ \\
\hline B-419-038 & 24-Aug-11 & B-419-038-2F & SO & 2 & Cesium 137 & 1.08 & $\mathrm{pCi} / \mathrm{g}$ & & 5.82 & 0.16 & $\mathrm{Y}$ & $\mathrm{N}$ \\
\hline B-419-038 & 24-Aug-11 & B-419-038-2F & SO & 2 & Cobalt 56 & 1.12 & $\mathrm{pCi} / \mathrm{g}$ & & 0.95 & 0.0547 & $\mathrm{Y}$ & $\mathrm{Y}$ \\
\hline B-419-038 & 24-Aug-11 & B-419-038-2F & SO & 2 & Curium 242 & 0.24 & $\mathrm{pCi} / \mathrm{g}$ & & 3.41 & 0.0143 & $\mathrm{Y}$ & $\mathrm{N}$ \\
\hline B-419-038 & 24-Aug-11 & B-419-038-2F & SO & 2 & Gross alpha & 262 & $\mathrm{pCi} / \mathrm{g}$ & & $\mathrm{NE}$ & 3.38 & $\mathrm{Y}$ & $\mathrm{N}$ \\
\hline B-419-038 & 24-Aug-11 & B-419-038-2F & SO & 2 & Gross beta & 9.8 & $\mathrm{pCi} / \mathrm{g}$ & & $\mathrm{NE}$ & 4.47 & $\mathrm{Y}$ & $\mathrm{N}$ \\
\hline B-419-038 & 24-Aug-11 & B-419-038-2F & SO & 2 & Lead 212 & 0.88 & $\mathrm{pCi} / \mathrm{g}$ & & 5,330 & 0.938 & $\mathrm{Y}$ & $\mathrm{N}$ \\
\hline B-419-038 & 24-Aug-11 & B-419-038-2F & SO & 2 & Plutonium 238 & 1.07 & $\mathrm{pCi} / \mathrm{g}$ & & 14.4 & 0.0355 & $\mathrm{Y}$ & $\mathrm{N}$ \\
\hline B-419-038 & 24-Aug-11 & B-419-038-2F & SO & 2 & Strontium 90 & 1.15 & $\mathrm{pCi} / \mathrm{g}$ & & 38 & 0.264 & $\mathrm{Y}$ & $\mathrm{N}$ \\
\hline B-419-038 & 24-Aug-11 & B-419-038-2F & SO & 2 & Thallium 208 & 0.31 & $\mathrm{pCi} / \mathrm{g}$ & & 33,600 & 0.371 & $\mathrm{Y}$ & $\mathrm{N}$ \\
\hline B-419-038 & 24-Aug-11 & B-419-038-2F & SO & 2 & Thorium 228 & 0.83 & $\mathrm{pCi} / \mathrm{g}$ & & 109 & 0.991 & $\mathrm{Y}$ & $\mathrm{N}$ \\
\hline B-419-038 & 24-Aug-11 & B-419-038-2F & SO & 2 & Tritium & 192 & $\mathrm{pCi} / \mathrm{g}$ & $\mathrm{J}$ & 1.27 & 0.106 & $\mathrm{Y}$ & $\mathrm{Y}$ \\
\hline B-419-038 & 24-Aug-11 & B-419-038-2F & SO & 2 & Uranium 234 and & 2.7 & $\mathrm{pCi} / \mathrm{g}$ & & 25.5 & 0.76 & $\mathrm{Y}$ & $\mathrm{N}$ \\
\hline B-419-038 & 24-Aug-11 & B-419-038-2F & SO & 2 & Uranium 235 and & 0.129 & $\mathrm{pCi} / \mathrm{g}$ & & 31.3 & 0.0904 & $\mathrm{Y}$ & $\mathrm{N}$ \\
\hline B-419-038 & 24-Aug-11 & B-419-038-2F & SO & 2 & Uranium 238 & 1.45 & $\mathrm{pCi} / \mathrm{g}$ & & 33 & 0.717 & $\mathrm{Y}$ & $\mathrm{N}$ \\
\hline B-419-038 & 24-Aug-11 & B-419-038-2F & SO & 2 & PCB 1254 & 2000 & ug/kg & $\mathrm{D}$ & 740 & $\mathrm{NE}$ & $\mathrm{N}$ & $\mathrm{Y}$ \\
\hline B-419-038 & 24-Aug-11 & B-419-038-DUP-2.5F & SO & 2.5 & Beryllium & 40 & $\mathrm{mg} / \mathrm{kg}$ & & 2,000 & 0.55 & $\mathrm{Y}$ & $\mathrm{N}$ \\
\hline B-419-038 & 24-Aug-11 & B-419-038-DUP-2.5F & SO & 2.5 & Cadmium & 4 & $\mathrm{mg} / \mathrm{kg}$ & & 800 & 0.55 & $\mathrm{Y}$ & $\mathrm{N}$ \\
\hline B-419-038 & 24-Aug-11 & B-419-038-DUP-2.5F & SO & 2.5 & Chromium & 300 & $\mathrm{mg} / \mathrm{kg}$ & $\mathrm{L}$ & $1,500,000(1)$ & 48.9 & $\mathrm{Y}$ & $\mathrm{N}$ \\
\hline B-419-038 & 24-Aug-11 & B-419-038-DUP-2.5F & SO & 2.5 & Copper & 200 & $\mathrm{mg} / \mathrm{kg}$ & & 41,000 & 35.8 & $\mathrm{Y}$ & $\mathrm{N}$ \\
\hline B-419-038 & 24-Aug-11 & B-419-038-DUP-2.5F & SO & 2.5 & Diesel Fuel & 29 & $\mathrm{mg} / \mathrm{kg}$ & & 180 & NE & $\mathrm{N}$ & $\mathrm{N}$ \\
\hline B-419-038 & 24-Aug-11 & B-419-038-DUP-2.5F & SO & 2.5 & Hexavalent & 12 & $\mathrm{mg} / \mathrm{kg}$ & $\mathrm{D}$ & 5.6 & 2.2 & $\mathrm{Y}$ & $\mathrm{Y}$ \\
\hline B-419-038 & 24-Aug-11 & B-419-038-DUP-2.5F & SO & 2.5 & Lead & 230 & $\mathrm{mg} / \mathrm{kg}$ & DO & 800 & 14.3 & $\mathrm{Y}$ & $\mathrm{N}$ \\
\hline B-419-038 & 24-Aug-11 & B-419-038-DUP-2.5F & SO & 2.5 & Mercury & 15 & $\mathrm{mg} / \mathrm{kg}$ & DO & 43 & 0.0759 & $\mathrm{Y}$ & $\mathrm{N}$ \\
\hline B-419-038 & 24-Aug-11 & B-419-038-DUP-2.5F & SO & 2.5 & Zinc & 170 & $\mathrm{mg} / \mathrm{kg}$ & & 310,000 & 69.8 & $\mathrm{Y}$ & $\mathrm{N}$ \\
\hline B-419-038 & 24-Aug-11 & B-419-038-DUP-2.5F & SO & 2.5 & Barium & 0.7 & $\mathrm{mg} / \mathrm{L}$ & & NA & NA & NA & NA \\
\hline
\end{tabular}


US EPA

Preliminary Bldg

Industrial Screening

background

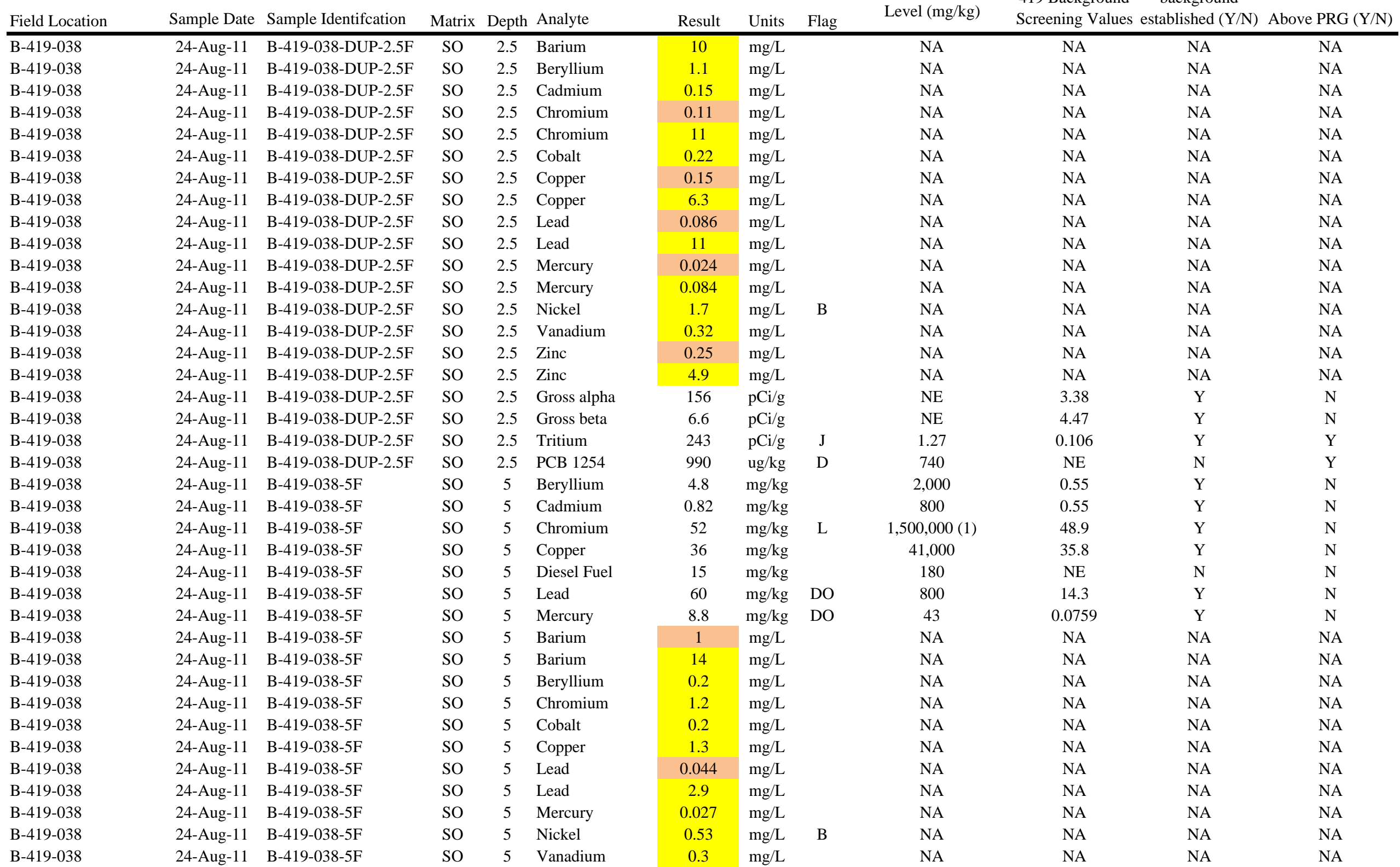


US EPA

Preliminary Bldg

Industrial Screening

background

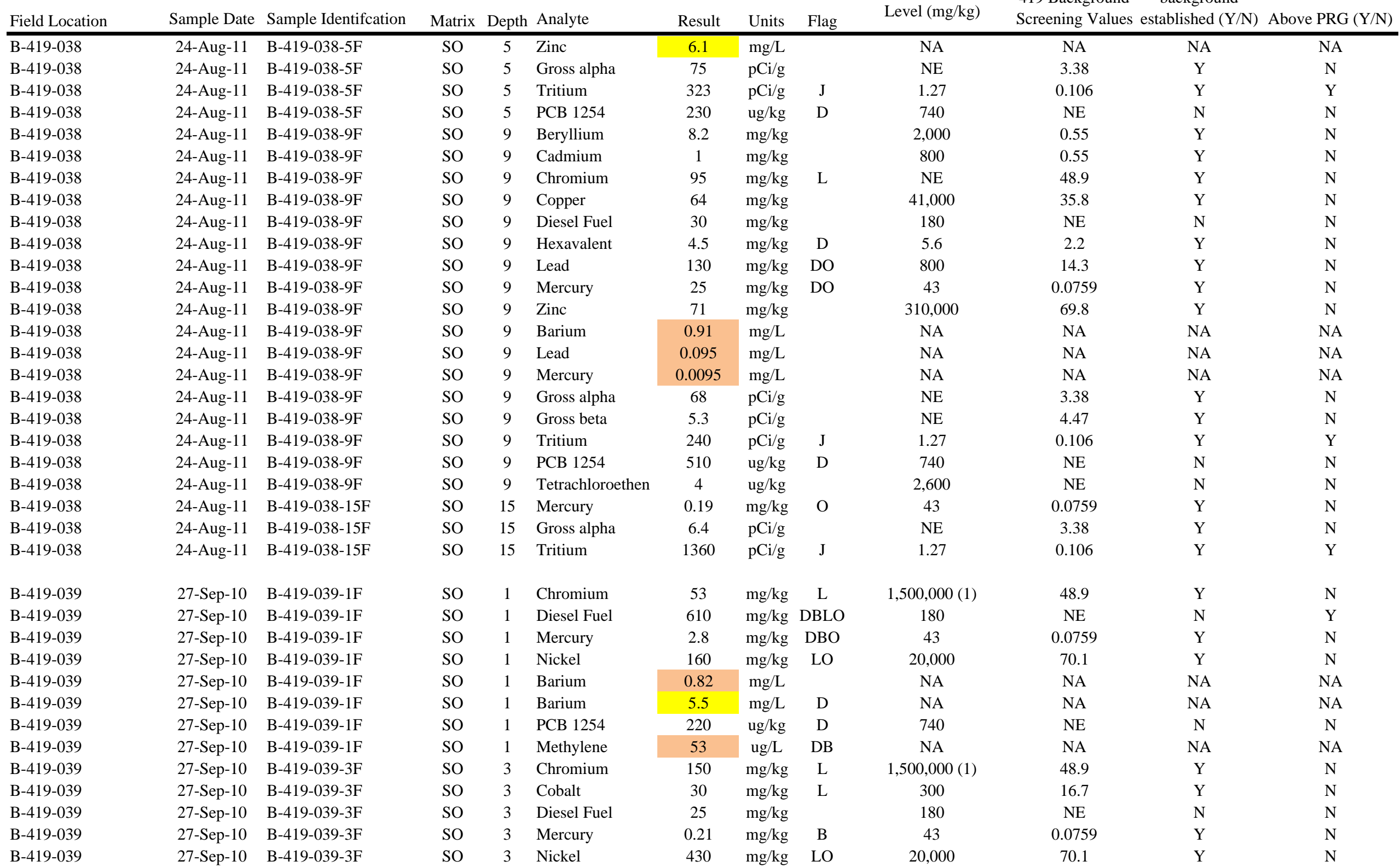


US EPA

Preliminary Bldg

Industrial Screening $\quad$ Plim

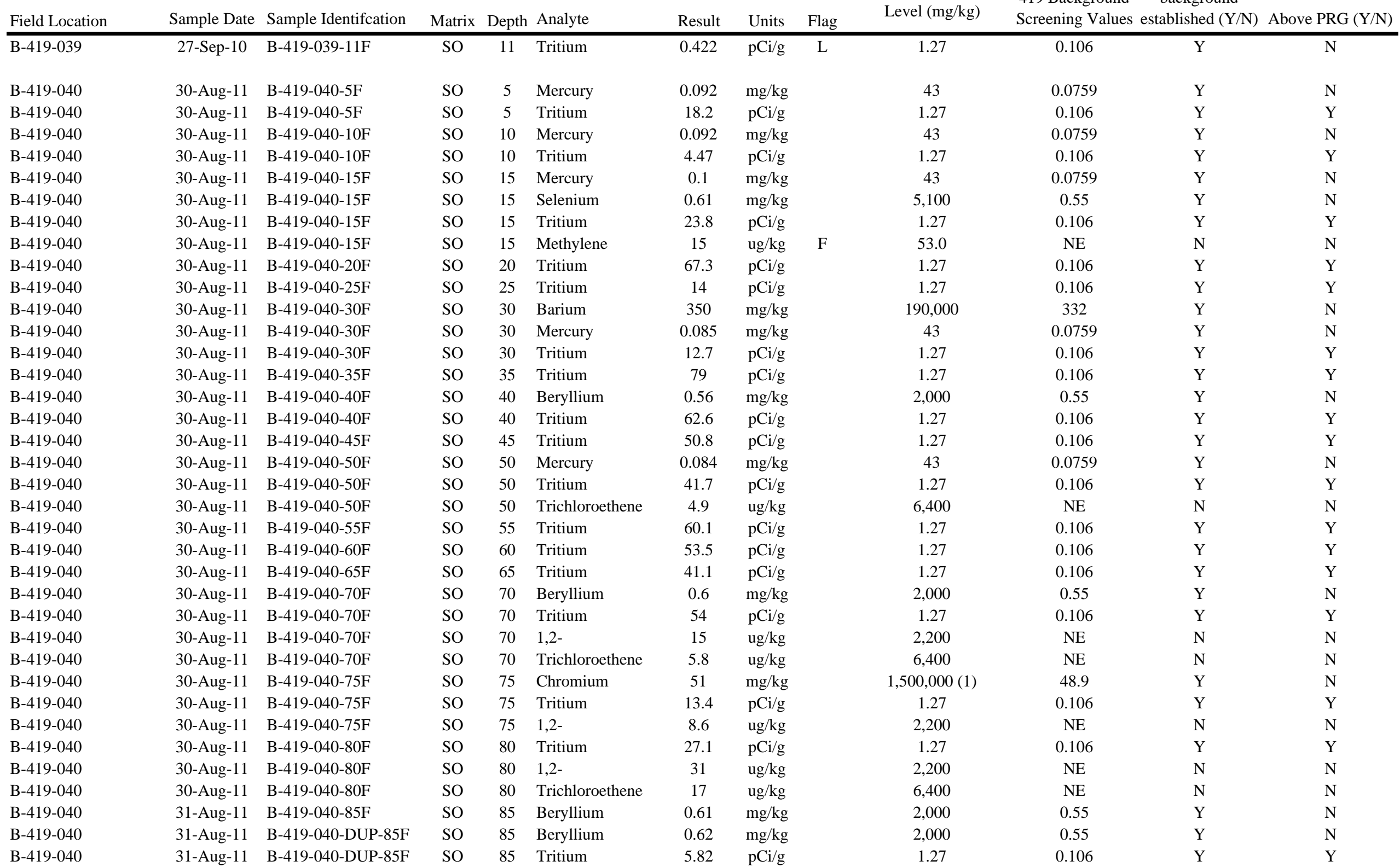


US EPA

Preliminary Bldg

Industrial Screening 419 Bacround

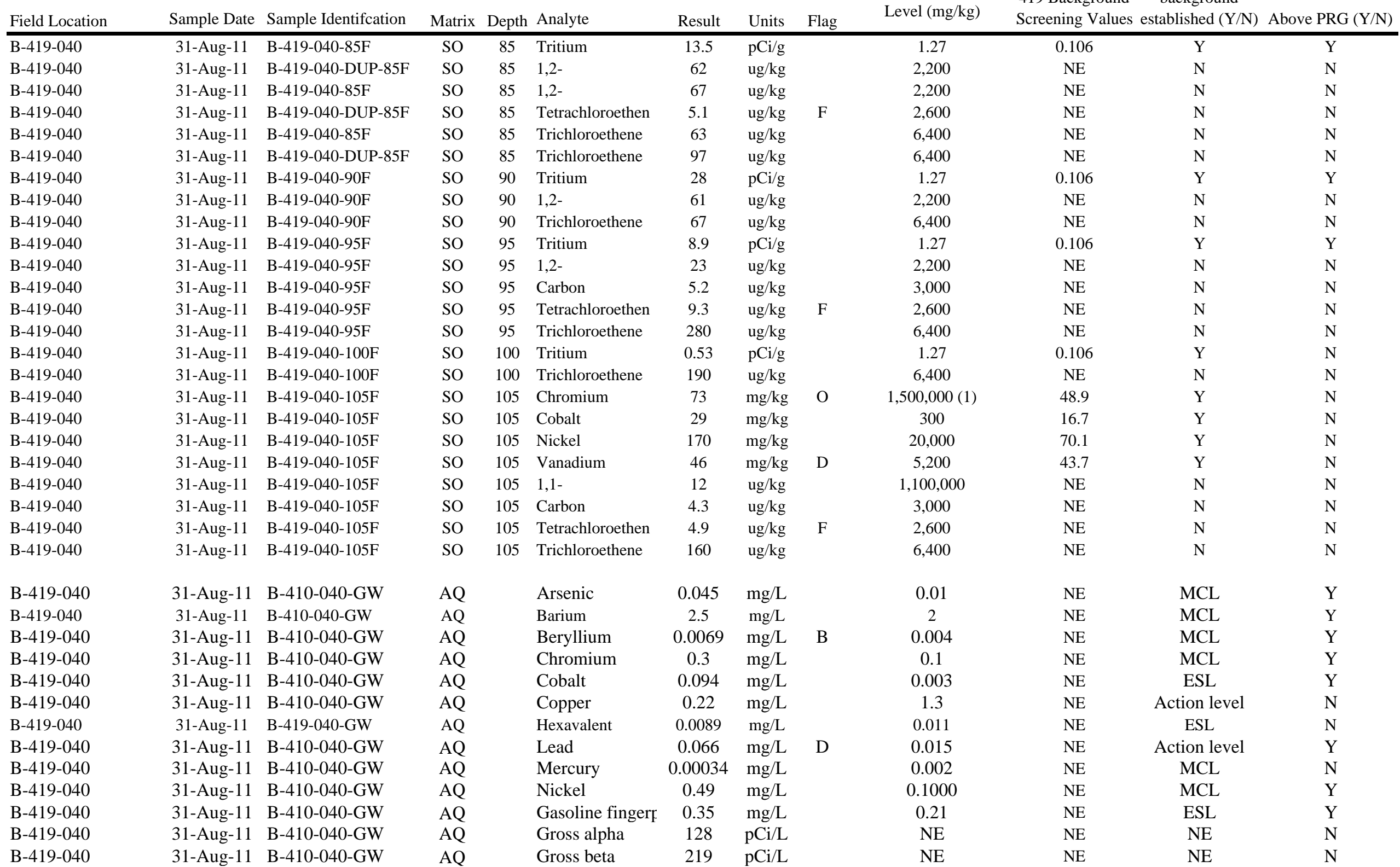


US EPA

Preliminary Bldg

Industrial Screening

Level (mg/kg)

Background baund

\begin{tabular}{|c|c|c|c|c|c|c|c|c|c|c|c|c|}
\hline Field Location & Sample Date & Sample Identifcation & Matrix & Depth & Analyte & Result & Units & Flag & Level (mg/kg) & Screening Values & established (Y/N) & Above PRG (Y/N) \\
\hline B-419-040 & 31-Aug-11 & B-410-040-GW & $\mathrm{AQ}$ & & Tritium & 59700 & $\overline{\mathrm{pCi} / \mathrm{L}}$ & & 20,000 & $\mathrm{NE}$ & $\overline{M C L}$ & $\bar{Y}$ \\
\hline B-419-040 & 31-Aug-11 & B-410-040-GW & $\mathrm{AQ}$ & & 1,1-Dichloroethı & 65 & ug/L & $\mathrm{D}$ & 6 & NE & MCL & $\mathrm{Y}$ \\
\hline B-419-040 & 31-Aug-11 & B-410-040-GW & AQ & & 1,2-Dichloroethi & 110 & ug/L & $\mathrm{D}$ & 5 & NE & MCL & $\mathrm{Y}$ \\
\hline B-419-041 & 6-Sep-11 & B-419-041-5F & SO & 5 & Tritium & 19.8 & $\mathrm{pCi} / \mathrm{g}$ & $\mathrm{L}$ & 1.27 & 0.106 & $\mathrm{Y}$ & $\mathrm{Y}$ \\
\hline B-419-041 & 6-Sep-11 & B-419-041-10F & SO & 10 & Mercury & 0.11 & $\mathrm{mg} / \mathrm{kg}$ & & 43 & 0.0759 & $\mathrm{Y}$ & $\mathrm{N}$ \\
\hline B-419-041 & 6-Sep-11 & B-419-041-10F & SO & 10 & Tritium & 19.9 & $\mathrm{pCi} / \mathrm{g}$ & $\mathrm{L}$ & 1.27 & 0.106 & $\mathrm{Y}$ & $\mathrm{Y}$ \\
\hline B-419-041 & 6-Sep-11 & B-419-041-15F & SO & 15 & Chromium & 72 & $\mathrm{mg} / \mathrm{kg}$ & & $1,500,000(1)$ & 48.9 & $\mathrm{Y}$ & $\mathrm{N}$ \\
\hline B-419-041 & 6-Sep-11 & B-419-041-15F & SO & 15 & Tritium & 116 & $\mathrm{pCi} / \mathrm{g}$ & $\mathrm{L}$ & 1.27 & 0.106 & $\mathrm{Y}$ & $\mathrm{Y}$ \\
\hline B-419-041 & 6-Sep-11 & B-419-041-DUP-20F & SO & 20 & Mercury & 0.077 & $\mathrm{mg} / \mathrm{kg}$ & & 43 & 0.0759 & $\mathrm{Y}$ & $\mathrm{N}$ \\
\hline B-419-041 & 6-Sep-11 & B-419-041-20F & SO & 20 & Mercury & 0.082 & $\mathrm{mg} / \mathrm{kg}$ & & 43 & 0.0759 & $\mathrm{Y}$ & $\mathrm{N}$ \\
\hline B-419-041 & 6-Sep-11 & B-419-041-20F & SO & 20 & Tritium & 990 & $\mathrm{pCi} / \mathrm{g}$ & $\mathrm{L}$ & 1.27 & 0.106 & $\mathrm{Y}$ & $\mathrm{Y}$ \\
\hline B-419-041 & 6-Sep-11 & B-419-041-DUP-20F & SO & 20 & Tritium & 1910 & $\mathrm{pCi} / \mathrm{g}$ & $\mathrm{L}$ & 1.27 & 0.106 & $\mathrm{Y}$ & $\mathrm{Y}$ \\
\hline B-419-041 & 6-Sep-11 & B-419-041-25F & SO & 25 & Tritium & 359 & $\mathrm{pCi} / \mathrm{g}$ & $\mathrm{L}$ & 1.27 & 0.106 & $\mathrm{Y}$ & $\mathrm{Y}$ \\
\hline B-419-041 & 6-Sep-11 & B-419-041-30F & SO & 30 & Tritium & 890 & $\mathrm{pCi} / \mathrm{g}$ & $\mathrm{L}$ & 1.27 & 0.106 & $\mathrm{Y}$ & $\mathrm{Y}$ \\
\hline B-419-041 & 6-Sep-11 & B-419-041-40F & SO & 40 & Tritium & 468 & $\mathrm{pCi} / \mathrm{g}$ & $\mathrm{L}$ & 1.27 & 0.106 & $\mathrm{Y}$ & $\mathrm{Y}$ \\
\hline B-419-041 & 6-Sep-11 & B-419-041-45F & SO & 45 & Mercury & 0.13 & $\mathrm{mg} / \mathrm{kg}$ & & 43 & 0.0759 & $\mathrm{Y}$ & $\mathrm{N}$ \\
\hline B-419-041 & 6-Sep-11 & B-419-041-45F & SO & 45 & Tritium & 277 & $\mathrm{pCi} / \mathrm{g}$ & $\mathrm{L}$ & 1.27 & 0.106 & $\mathrm{Y}$ & $\mathrm{Y}$ \\
\hline B-419-041 & 6-Sep-11 & B-419-041-50F & SO & 50 & Tritium & 311 & $\mathrm{pCi} / \mathrm{g}$ & $\mathrm{L}$ & 1.27 & 0.106 & $\mathrm{Y}$ & $\mathrm{Y}$ \\
\hline B-419-041 & 6-Sep-11 & B-419-041-55F & SO & 55 & Tritium & 220 & $\mathrm{pCi} / \mathrm{g}$ & $\mathrm{L}$ & 1.27 & 0.106 & $\mathrm{Y}$ & $\mathrm{Y}$ \\
\hline B-419-041 & 6-Sep-11 & B-419-041-60F & SO & 60 & Tritium & 117 & $\mathrm{pCi} / \mathrm{g}$ & $\mathrm{L}$ & 1.27 & 0.106 & $\mathrm{Y}$ & $\mathrm{Y}$ \\
\hline B-419-041 & 6-Sep-11 & B-419-041-65F & SO & 65 & Tritium & 57.2 & $\mathrm{pCi} / \mathrm{g}$ & $\mathrm{L}$ & 1.27 & 0.106 & $\mathrm{Y}$ & $\mathrm{Y}$ \\
\hline B-419-041 & 7-Sep-11 & B-419-041-70F & SO & 70 & Tritium & 102 & $\mathrm{pCi} / \mathrm{g}$ & LO & 1.27 & 0.106 & $\mathrm{Y}$ & $\mathrm{Y}$ \\
\hline B-419-041 & 7-Sep-11 & B-419-041-75F & SO & 75 & Tritium & 40.5 & $\mathrm{pCi} / \mathrm{g}$ & $\mathrm{L}$ & 1.27 & 0.106 & $\mathrm{Y}$ & $\mathrm{Y}$ \\
\hline B-419-041 & 7-Sep-11 & B-419-041-75F & SO & 75 & $1,2-$ & 10 & $\mathrm{ug} / \mathrm{kg}$ & & 2,200 & NE & $\mathrm{N}$ & $\mathrm{N}$ \\
\hline B-419-041 & 7-Sep-11 & B-419-041-75F & SO & 75 & Acetone & 18 & ug/kg & & $630,000,000$ & $\mathrm{NE}$ & $\mathrm{N}$ & $\mathrm{N}$ \\
\hline B-419-041 & 7-Sep-11 & B-419-041-75F & SO & 75 & Trichloroethene & 6.3 & ug/kg & & 6,400 & $\mathrm{NE}$ & $\mathrm{N}$ & $\mathrm{N}$ \\
\hline B-419-041 & 7-Sep-11 & B-419-041-80F & SO & 80 & Tritium & 40.4 & $\mathrm{pCi} / \mathrm{g}$ & $\mathrm{L}$ & 1.27 & 0.106 & $\mathrm{Y}$ & $\mathrm{Y}$ \\
\hline B-419-041 & 7-Sep-11 & B-419-041-DUP-80F & SO & 80 & Tritium & 62.8 & $\mathrm{pCi} / \mathrm{g}$ & $\mathrm{L}$ & 1.27 & 0.106 & $\mathrm{Y}$ & $\mathrm{Y}$ \\
\hline B-419-041 & 7-Sep-11 & B-419-041-80F & SO & 80 & $1,2-$ & 17 & ug/kg & & 2,200 & NE & $\mathrm{N}$ & $\mathrm{N}$ \\
\hline B-419-041 & 7-Sep-11 & B-419-041-DUP-80F & SO & 80 & $1,2-$ & 26 & ug/kg & & 2,200 & NE & $\mathrm{N}$ & $\mathrm{N}$ \\
\hline B-419-041 & 7-Sep-11 & B-419-041-85F & SO & 85 & Beryllium & 0.58 & $\mathrm{mg} / \mathrm{kg}$ & & 2,000 & 0.55 & $\mathrm{Y}$ & $\mathrm{N}$ \\
\hline B-419-041 & 7-Sep-11 & B-419-041-85F & SO & 85 & Tritium & 19.6 & $\mathrm{pCi} / \mathrm{g}$ & $\mathrm{L}$ & 1.27 & 0.106 & $\mathrm{Y}$ & $\mathrm{Y}$ \\
\hline
\end{tabular}


US EPA

Preliminary Bldg

Field Location

Sample Date Sample Identifcation

Matrix Depth Analyte

B-419-041

B-419-041

B-419-041

B-419-041

B-419-041

B-419-041

B-419-041

B-419-041

B-419-041

B-419-041

B-419-041

B-419-041

B-419-041

B-419-041

B-419-041

B-419-041

B-419-041

B-419-041

B-419-041

B-419-041

B-419-041

B-419-041

B-419-041

B-419-041

B-419-041

B-419-041

B-419-041

B-419-042A

B-419-042A

B-419-042A

B-419-042A

B-419-042A

B-419-042A

B-419-042A

B-419-042A

$\begin{array}{lllll}\text { 7-Sep-11 } & \text { B-419-041-85F } & \text { SO } & 85 & \text { 1,2- }\end{array}$

7-Sep-11 B-419-041-85F

7-Sep-11 B-419-041-90F

7-Sep-11 B-419-041-90F

7-Sep-11 B-419-041-90F

7-Sep-11 B-419-041-95F

7-Sep-11 B-419-041-95F

7-Sep-11 B-419-041-95F

7-Sep-11 B-419-041-100F

7-Sep-11 B-419-041-100F

7-Sep-11 B-419-041-100F

7-Sep-11 B-419-041-100F

7-Sep-11 B-419-041-GW

7-Sep-11 B-419-041-GW

7-Sep-11 B-419-041-GW

7-Sep-11 B-419-041-GW

7-Sep-11 B-419-041-GW

7-Sep-11 B-419-041-GW

7-Sep-11 B-419-041-GW

7-Sep-11 B-419-041-GW

7-Sep-11 B-419-041-GW

7-Sep-11 B-419-041-GW

7-Sep-11 B-419-041-GW

7-Sep-11 B-419-041-GW

7-Sep-11 B-419-041-GW

7-Sep-11 B-419-041-GW

15-Aug-11 B-419-042A-1F

15-Aug-11 B-419-042A-1F

15-Aug-11 B-419-042A-1F

15-Aug-11 B-419-042A-1F

15-Aug-11 B-419-042A-1F

15-Aug-11 B-419-042A-1F

15-Aug-11 B-419-042A-1F

15-Aug-11 B-419-042A-1F
7-Sep-11 B-419-041-100F

SO 100 Tritium
Industrial Screening

\section{Trichloroethene}

SO 90 Tritium

SO $90 \quad 1,2-$

SO 90 Trichloroethene

SO 95 Tritium

$\mathrm{SO} \quad 95 \quad 1,2-$

SO 95 Trichloroethene

SO $1001,1-$

SO 100 Tetrachloroethen

SO 100 Trichloroethene

\section{AQ}

AQ

AQ

AQ

AQ

AQ

AQ

AQ

AQ

AQ

AQ

AQ

AQ

AQ

Arsenic
Barium
Chromium
Cobalt
Copper
Hexavalent
Lead
Nickel
Gasoline
Gross alpha
Gross beta
Tritium
Tetrachloroe
Trichloroeth

Barium
Beryllium
Cadmium
Cobalt
Diesel Fuel
Gasoline
Lead
Mercury

$\begin{array}{lll}\text { SO } & 1 & \text { Barium } \\ \text { SO } & 1 & \text { Beryllium } \\ \text { SO } & 1 & \text { Cadmium } \\ \text { SO } & 1 & \text { Cobalt } \\ \text { SO } & 1 & \text { Diesel Fuel } \\ \text { SO } & 1 & \text { Gasoline } \\ \text { SO } & 1 & \text { Lead } \\ \text { SO } & 1 & \text { Mercury }\end{array}$

SO 100 Carbon

Result Units Flag

Level (mg/kg)

419 Background background

2,200

ug/kg

$\mathrm{ug} / \mathrm{kg}$

pCi/g

$\mathrm{ug} / \mathrm{kg}$

ug/kg

$\mathrm{pCi} / \mathrm{g}$

ug $/ \mathrm{kg}$

ug/kg

pCi/g

ug/kg

$\mathrm{ug} / \mathrm{kg}$

ug/kg

ug/kg

$0.023 \quad \mathrm{mg} / \mathrm{L}$

$\mathrm{mg} / \mathrm{L}$
$\mathrm{mg} / \mathrm{L}$

$\mathrm{mg} / \mathrm{L}$

$\begin{array}{cc}0.18 & \mathrm{mg} / \mathrm{L} \\ 0.058 & \mathrm{mg} / \mathrm{L}\end{array}$

$0.12 \mathrm{mg} / \mathrm{L}$

$0.0089 \mathrm{mg} / \mathrm{L}$

$0.038 \mathrm{mg} / \mathrm{L}$

$0.26 \mathrm{mg} / \mathrm{L}$

$0.36 \mathrm{mg} / \mathrm{L}$

$46 \quad \mathrm{pCi} / \mathrm{L}$

$83 \mathrm{pCi} / \mathrm{L}$

$59800 \quad \mathrm{pCi} / \mathrm{L}$

$\begin{array}{cc}53 & \mathrm{ug} / \mathrm{L} \\ 1700 & \mathrm{ug} / \mathrm{L}\end{array}$

$\begin{array}{cccc}460 & \mathrm{mg} / \mathrm{kg} & \mathrm{L} & 190,000 \\ 2.8 & \mathrm{mg} / \mathrm{kg} & & 2,000 \\ 2.6 & \mathrm{mg} / \mathrm{kg} & & 800 \\ 130 & \mathrm{mg} / \mathrm{kg} & & 300 \\ 170 & \mathrm{mg} / \mathrm{kg} & \mathrm{D} & 180 \\ 1.2 & \mathrm{mg} / \mathrm{kg} & & 180 \\ 80 & \mathrm{mg} / \mathrm{kg} & & 800 \\ 6.8 & \mathrm{mg} / \mathrm{kg} & \mathrm{D} & 43\end{array}$

1 Mercury 6.8

332
0.55
0.55
16.7
$\mathrm{NE}$
$\mathrm{NE}$
14.3
0.0759

Screening Values established (Y/N) Above PRG (Y/N)

\begin{tabular}{|c|c|c|c|}
\hline 2,200 & $\mathrm{NE}$ & $\mathrm{N}$ & $\mathrm{N}$ \\
\hline 6,400 & NE & $\mathrm{N}$ & $\mathrm{N}$ \\
\hline 1.27 & 0.106 & Y & $\mathrm{Y}$ \\
\hline 2,200 & NE & $\mathrm{N}$ & $\mathrm{N}$ \\
\hline 6,400 & NE & $\mathrm{N}$ & $\mathrm{N}$ \\
\hline 1.27 & 0.106 & Y & $\mathrm{Y}$ \\
\hline 2,200 & $\mathrm{NE}$ & $\mathrm{N}$ & $\mathrm{N}$ \\
\hline 6,400 & NE & $\mathrm{N}$ & $\mathrm{N}$ \\
\hline 1.27 & 0.106 & Y & $\mathrm{N}$ \\
\hline L,100,000 & $\mathrm{NE}$ & $\mathrm{N}$ & $\mathrm{N}$ \\
\hline 3,000 & NE & $\mathrm{N}$ & $\mathrm{N}$ \\
\hline 2,600 & $\mathrm{NE}$ & $\mathrm{N}$ & $\mathrm{N}$ \\
\hline 6,400 & NE & $\mathrm{N}$ & $\mathrm{N}$ \\
\hline 0.010 & $\mathrm{NE}$ & MCL & $\mathrm{Y}$ \\
\hline 2 & $\mathrm{NE}$ & MCL & $\mathrm{Y}$ \\
\hline 0.1 & $\mathrm{NE}$ & MCL & $\mathrm{Y}$ \\
\hline 0.003 & $\mathrm{NE}$ & ESL & $\mathrm{Y}$ \\
\hline 1.3 & $\mathrm{NE}$ & Action Level & $\mathrm{N}$ \\
\hline 0.011 & $\mathrm{NE}$ & ESL & $\mathrm{N}$ \\
\hline 0.015 & $\mathrm{NE}$ & Action Level & $\mathrm{Y}$ \\
\hline 0.1 & $\mathrm{NE}$ & MCL & $\mathrm{Y}$ \\
\hline 0.21 & $\mathrm{NE}$ & ESL & $\mathrm{Y}$ \\
\hline $\mathrm{NE}$ & $\mathrm{NE}$ & $\mathrm{NE}$ & $\mathrm{N}$ \\
\hline NE & $\mathrm{NE}$ & $\mathrm{NE}$ & $\mathrm{N}$ \\
\hline 20,000 & $\mathrm{NE}$ & MCL & $\mathrm{Y}$ \\
\hline 5 & $\mathrm{NE}$ & MCL & $\mathrm{Y}$ \\
\hline 5 & NE & MCL & Y \\
\hline
\end{tabular}

0.0759

$\begin{array}{ll}\mathrm{Y} & \mathrm{N} \\ \mathrm{Y} & \mathrm{N} \\ \mathrm{Y} & \mathrm{N} \\ \mathrm{Y} & \mathrm{N} \\ \mathrm{N} & \mathrm{N} \\ \mathrm{N} & \mathrm{N} \\ \mathrm{Y} & \mathrm{N} \\ \mathrm{Y} & \mathrm{N}\end{array}$


US EPA

Preliminary Bldg

Industrial Screening $\quad 41$ r

background

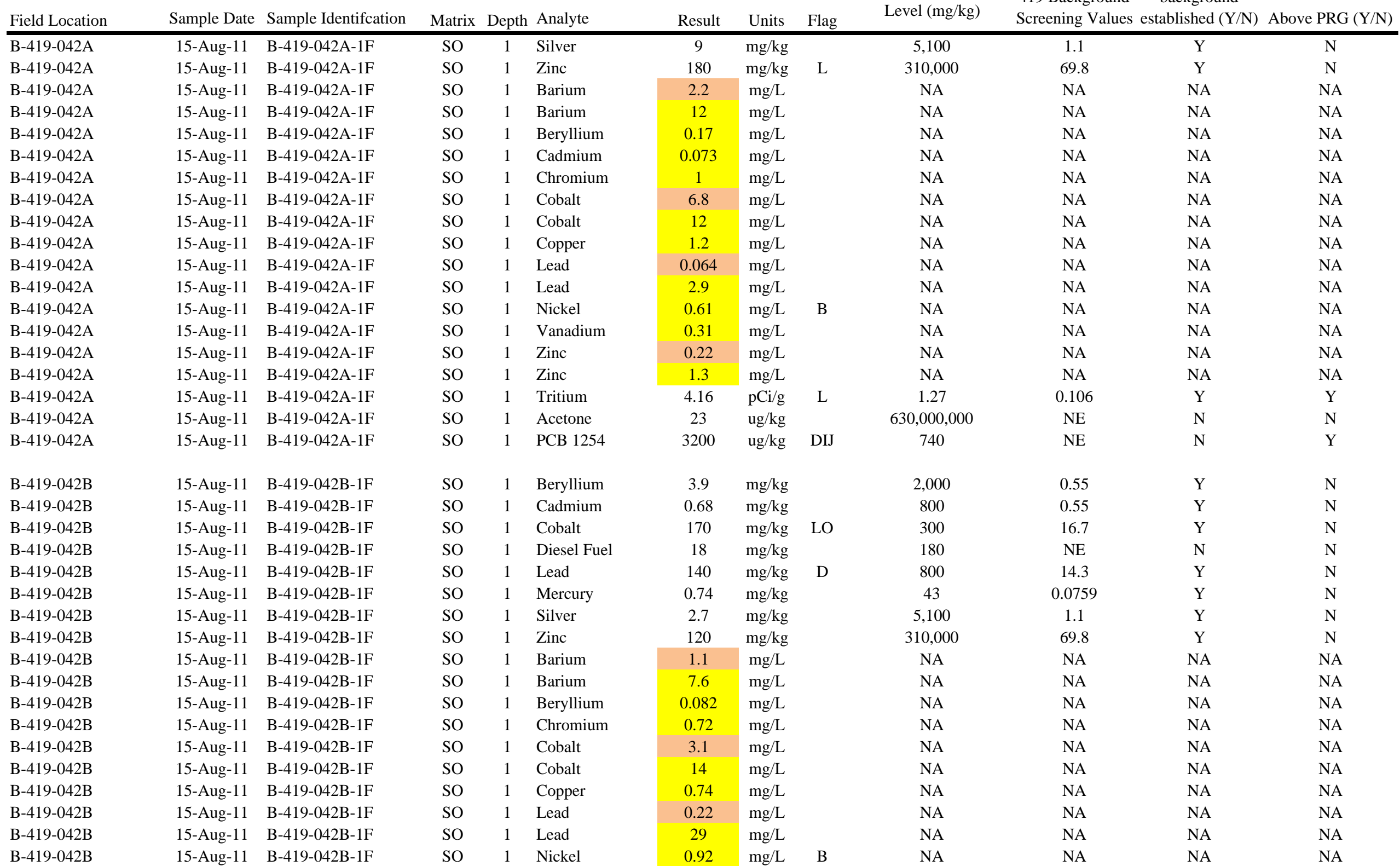


US EPA

Preliminary Bldg

Field Location Sample Date Sample Identifcation Matrix Depth Analyte

Result Units Flag

15-Aug-11 B-419-042B-1F $\quad$ SO 11 Vanadium

15-Aug-11 B-419-042B-1F

15-Aug-11 B-419-042B-1F

B-419-042B

B-419-042B

B-419-042B

B-419-042B

B-419-042B

B-419-043

B-419-043

B-419-043

B-419-043

B-419-043

B-419-043

B-419-043

B-419-043

B-419-043

B-419-043

B-419-043

B-419-043

B-419-044

B-419-044

B-419-044

B-419-044

B-419-045

B-419-045

B-419-045

B-419-046

B-419-046

B-419-046

B-419-046

B-419-046

B-419-046

B-419-046

B-419-046

B-419-046
15-Aug-11 B-419-042B-1F

15-Aug-11 B-419-042B-1F

15-Aug-11 B-419-042B-1F

15-Aug-11 B-419-043-1F

15-Aug-11 B-419-043-1F

15-Aug-11 B-419-043-1F

15-Aug-11 B-419-043-1F

15-Aug-11 B-419-043-1F

15-Aug-11 B-419-043-1F

15-Aug-11 B-419-043-1F

15-Aug-11 B-419-043-1F

15-Aug-11 B-419-043-1F

15-Aug-11 B-419-043-1F

15-Aug-11 B-419-043-1F

15-Aug-11 B-419-043-1F

15-Aug-11 B-419-044-1F

15-Aug-11 B-419-044-1F

15-Aug-11 B-419-044-1F

15-Aug-11 B-419-044-1F

15-Aug-11 B-419-045-1F

15-Aug-11 B-419-045-1F

15-Aug-11 B-419-045-1F

24-Aug-11 B-419-046-1F

24-Aug-11 B-419-046-1F

24-Aug-11 B-419-046-1F

24-Aug-11 B-419-046-1F

24-Aug-11 B-419-046-1F

24-Aug-11 B-419-046-1F

24-Aug-11 B-419-046-1F

24-Aug-11 B-419-046-1F

24-Aug-11 B-419-046-1F

$\begin{array}{lllclll}\text { SO } & 1 & \text { Vanadium } & 0.42 & \mathrm{mg} / \mathrm{L} & \\ \text { SO } & 1 & \text { Zinc } & 0.3 & \mathrm{mg} / \mathrm{L} & \\ \text { SO } & 1 & \text { Zinc } & 3.8 & \mathrm{mg} / \mathrm{L} & \\ \text { SO } & 1 & \text { Tritium } & 0.63 & \mathrm{pCi} / \mathrm{g} & \\ \text { SO } & 1 & \text { Acetone } & 40 & \mathrm{ug} / \mathrm{kg} & \\ \text { SO } & 1 & \text { PCB 1254 } & 5000 & \mathrm{ug} / \mathrm{kg} & \text { DIJ }\end{array}$

\section{SO 1 Antimony}

SO 1 Cobalt

SO 1 Copper

SO 1 Lead

SO 1 Molybdenum

SO 1 Zinc

SO 1 Benzo(a)anthrace

SO 1 Benzo(b)fluorant

SO 1 Chrysene

SO 1 Fluoranthene

SO 1 Phenanthrene

SO 1 Pyrene

\section{SO 1 Cobalt}

SO 1 Lead

SO 1 Mercury

SO 1 Silver

\section{SO 1 Cadmium}

SO 1 Cobalt

SO 1 Silver

\section{SO 1 Beryllium}

SO 1 Lead

SO 1 Barium

SO 1 Barium

SO 1 Beryllium

SO 1 Chromium

SO 1 Cobalt

SO 1 Copper
SO 1 Mercury
Industrial Screening

Level (mg/kg)

419 Back

$\begin{array}{llll}\text { NA } & \text { NA } & \text { NA } & \text { NA }\end{array}$

NA

NA

1.27

$630,000,000$

740

NA

NA

0.106

$\mathrm{NE}$

$\mathrm{NE}$

2.2

410
300

41,000

800

5,100

310,000

2,100

2,100

210,000

$22,000,000$

$\mathrm{NE}$

$17,000,000$

NA NA

NA

NA

$\mathrm{N}$

$\mathrm{N}$

$\mathrm{N}$

$\mathrm{Y}$

$\begin{array}{cccc}50 & \mathrm{mg} / \mathrm{kg} & \mathrm{LO} & 300 \\ 67 & \mathrm{mg} / \mathrm{kg} & & 41,000 \\ 34 & \mathrm{mg} / \mathrm{kg} & \mathrm{D} & 800 \\ 7 & \mathrm{mg} / \mathrm{kg} & & 5,100 \\ 97 & \mathrm{mg} / \mathrm{kg} & & 310,000 \\ 370 & \mathrm{ug} / \mathrm{kg} & 2,100 \\ 420 & \mathrm{ug} / \mathrm{kg} & 2,100 \\ 410 & \mathrm{ug} / \mathrm{kg} & 210,000 \\ 840 & \mathrm{ug} / \mathrm{kg} & 22,000,000 \\ 770 & \mathrm{ug} / \mathrm{kg} & \mathrm{NE} \\ 740 & \mathrm{ug} / \mathrm{kg} & & 17,000,000\end{array}$

\section{7}

35.8

14.3

1.1

1.1
69.8

NE

NE

NE

NE

NE

NE

$\begin{array}{cc}300 & 16.7 \\ 800 & 14.3 \\ 43 & 0.0759\end{array}$

5,100

1.1

0.55

800

300
5,100

16.7

1.1

0.55

2,000

800

43

NA

NA

NA

NA

NA

NA
14.3

0.0759

NA

NA

NA

NA

$Y$

Y

Y

$\mathrm{Y}$
$\mathrm{Y}$

Y

Y

N

N

N

$\mathrm{N}$
$\mathrm{N}$

N

Y

Y

Y

Y

$\mathrm{Y}$
$\mathrm{Y}$

N$$
\text { N }
$$

Y N

Y

$\mathrm{Y} \quad \mathrm{N}$

NA

NA

NA

NA

NA

NA 
US EPA

Preliminary Bldg

Industrial Screening

Level (mg/kg)

background

\begin{tabular}{|c|c|c|c|c|c|c|c|c|c|c|c|c|}
\hline Field Location & Sample Date & Sample Identifcation & Matrix & Depth & Analyte & Result & Units & Flag & Level (mg/kg) & Screening Values & established (Y/N) & Above PRG (Y/N) \\
\hline B-419-046 & 24-Aug-11 & B-419-046-1F & SO & 1 & Lead & 0.56 & $\mathrm{mg} / \mathrm{L}$ & & NA & NA & NA & NA \\
\hline B-419-046 & 24-Aug-11 & B-419-046-1F & SO & 1 & Lead & 13 & $\mathrm{mg} / \mathrm{L}$ & & NA & NA & NA & NA \\
\hline B-419-046 & 24-Aug-11 & B-419-046-1F & SO & 1 & Zinc & 1.7 & $\mathrm{mg} / \mathrm{L}$ & & NA & NA & NA & NA \\
\hline B-419-046 & 24-Aug-11 & B-419-046-1F & SO & 1 & Gross beta & 6.6 & $\mathrm{pCi} / \mathrm{g}$ & & $\mathrm{NE}$ & 4.47 & $\mathrm{Y}$ & $\mathrm{N}$ \\
\hline B-419-046 & 24-Aug-11 & B-419-046-1F & SO & 1 & Tritium & 232 & $\mathrm{pCi} / \mathrm{g}$ & $\mathrm{J}$ & 1.27 & 0.106 & $\mathrm{Y}$ & $\mathrm{Y}$ \\
\hline B-419-046 & 24-Aug-11 & B-419-046-1F & SO & 1 & РCB 1254 & 97 & ug/kg & & 740 & NE & $\mathrm{N}$ & $\mathrm{N}$ \\
\hline B-419-046 & 24-Aug-11 & B-419-046-5F & SO & 5 & Lead & 50 & $\mathrm{mg} / \mathrm{kg}$ & DO & 800 & 14.3 & $\mathrm{Y}$ & $\mathrm{N}$ \\
\hline B-419-046 & 24-Aug-11 & B-419-046-5F & SO & 5 & Mercury & 0.99 & $\mathrm{mg} / \mathrm{kg}$ & $\mathrm{O}$ & 43 & 0.0759 & $\mathrm{Y}$ & $\mathrm{N}$ \\
\hline B-419-046 & 24-Aug-11 & B-419-046-5F & SO & 5 & Barium & 0.89 & $\mathrm{mg} / \mathrm{L}$ & & NA & NA & NA & NA \\
\hline B-419-046 & 24-Aug-11 & B-419-046-5F & SO & 5 & Barium & 8.8 & $\mathrm{mg} / \mathrm{L}$ & & NA & NA & NA & NA \\
\hline B-419-046 & 24-Aug-11 & B-419-046-5F & SO & 5 & Chromium & 0.17 & $\mathrm{mg} / \mathrm{L}$ & & NA & NA & NA & NA \\
\hline B-419-046 & 24-Aug-11 & B-419-046-5F & SO & 5 & Cobalt & 0.14 & $\mathrm{mg} / \mathrm{L}$ & & NA & NA & NA & NA \\
\hline B-419-046 & 24-Aug-11 & B-419-046-5F & SO & 5 & Copper & 0.31 & $\mathrm{mg} / \mathrm{L}$ & & NA & NA & NA & NA \\
\hline B-419-046 & 24-Aug-11 & B-419-046-5F & SO & 5 & Lead & 0.097 & $\mathrm{mg} / \mathrm{L}$ & & NA & NA & NA & NA \\
\hline B-419-046 & 24-Aug-11 & B-419-046-5F & SO & 5 & Lead & 2.5 & $\mathrm{mg} / \mathrm{L}$ & & NA & NA & NA & NA \\
\hline B-419-046 & 24-Aug-11 & B-419-046-5F & SO & 5 & Zinc & 1.4 & $\mathrm{mg} / \mathrm{L}$ & & NA & NA & NA & NA \\
\hline B-419-046 & 24-Aug-11 & B-419-046-5F & SO & 5 & Gross alpha & 5.5 & $\mathrm{pCi} / \mathrm{g}$ & & $\mathrm{NE}$ & 3.38 & $\mathrm{Y}$ & $\mathrm{N}$ \\
\hline B-419-046 & 24-Aug-11 & B-419-046-5F & SO & 5 & Tritium & 367 & $\mathrm{pCi} / \mathrm{g}$ & $\mathrm{J}$ & 1.27 & 0.106 & $\mathrm{Y}$ & $\mathrm{Y}$ \\
\hline B-419-046 & 24-Aug-11 & B-419-046-10F & SO & 10 & Beryllium & 2.1 & $\mathrm{mg} / \mathrm{kg}$ & & 2,000 & 0.55 & $\mathrm{Y}$ & $\mathrm{N}$ \\
\hline B-419-046 & 24-Aug-11 & B-419-046-10F & SO & 10 & Cadmium & 1.2 & $\mathrm{mg} / \mathrm{kg}$ & & 800 & 0.55 & $\mathrm{Y}$ & $\mathrm{N}$ \\
\hline B-419-046 & 24-Aug-11 & B-419-046-10F & SO & 10 & Copper & 41 & $\mathrm{mg} / \mathrm{kg}$ & & 41,000 & 35.8 & $\mathrm{Y}$ & $\mathrm{N}$ \\
\hline B-419-046 & 24-Aug-11 & B-419-046-10F & SO & 10 & Lead & 330 & $\mathrm{mg} / \mathrm{kg}$ & DO & 800 & 14.3 & $\mathrm{Y}$ & $\mathrm{N}$ \\
\hline B-419-046 & 24-Aug-11 & B-419-046-10F & SO & 10 & Mercury & 3.6 & $\mathrm{mg} / \mathrm{kg}$ & DO & 43 & 0.0759 & $\mathrm{Y}$ & $\mathrm{N}$ \\
\hline B-419-046 & 24-Aug-11 & B-419-046-10F & SO & 10 & Barium & 0.73 & $\mathrm{mg} / \mathrm{L}$ & & NA & NA & NA & NA \\
\hline B-419-046 & 24-Aug-11 & B-419-046-10F & SO & 10 & Barium & 9.6 & $\mathrm{mg} / \mathrm{L}$ & & NA & NA & NA & NA \\
\hline B-419-046 & 24-Aug-11 & B-419-046-10F & SO & 10 & Beryllium & 0.05 & $\mathrm{mg} / \mathrm{L}$ & & NA & NA & NA & NA \\
\hline B-419-046 & 24-Aug-11 & B-419-046-10F & SO & 10 & Cadmium & 0.054 & $\mathrm{mg} / \mathrm{L}$ & & NA & NA & NA & NA \\
\hline B-419-046 & 24-Aug-11 & B-419-046-10F & SO & 10 & Chromium & 0.57 & $\mathrm{mg} / \mathrm{L}$ & & NA & NA & NA & NA \\
\hline B-419-046 & 24-Aug-11 & B-419-046-10F & SO & 10 & Cobalt & 0.16 & $\mathrm{mg} / \mathrm{L}$ & & NA & NA & NA & NA \\
\hline B-419-046 & 24-Aug-11 & B-419-046-10F & SO & 10 & Copper & 0.76 & $\mathrm{mg} / \mathrm{L}$ & & NA & NA & NA & NA \\
\hline B-419-046 & 24-Aug-11 & B-419-046-10F & SO & 10 & Lead & 0.27 & $\mathrm{mg} / \mathrm{L}$ & & NA & NA & NA & NA \\
\hline B-419-046 & 24-Aug-11 & B-419-046-10F & SO & 10 & Vanadium & 0.2 & $\mathrm{mg} / \mathrm{L}$ & & NA & NA & NA & NA \\
\hline B-419-046 & 24-Aug-11 & B-419-046-10F & SO & 10 & Zinc & 0.89 & $\mathrm{mg} / \mathrm{L}$ & & NA & NA & NA & NA \\
\hline
\end{tabular}


US EPA

Preliminary Bldg

Industrial Screening

Field Location Sample Date Sample Identifcation Matrix Depth Analyte

Level (mg/kg)

419 Background background

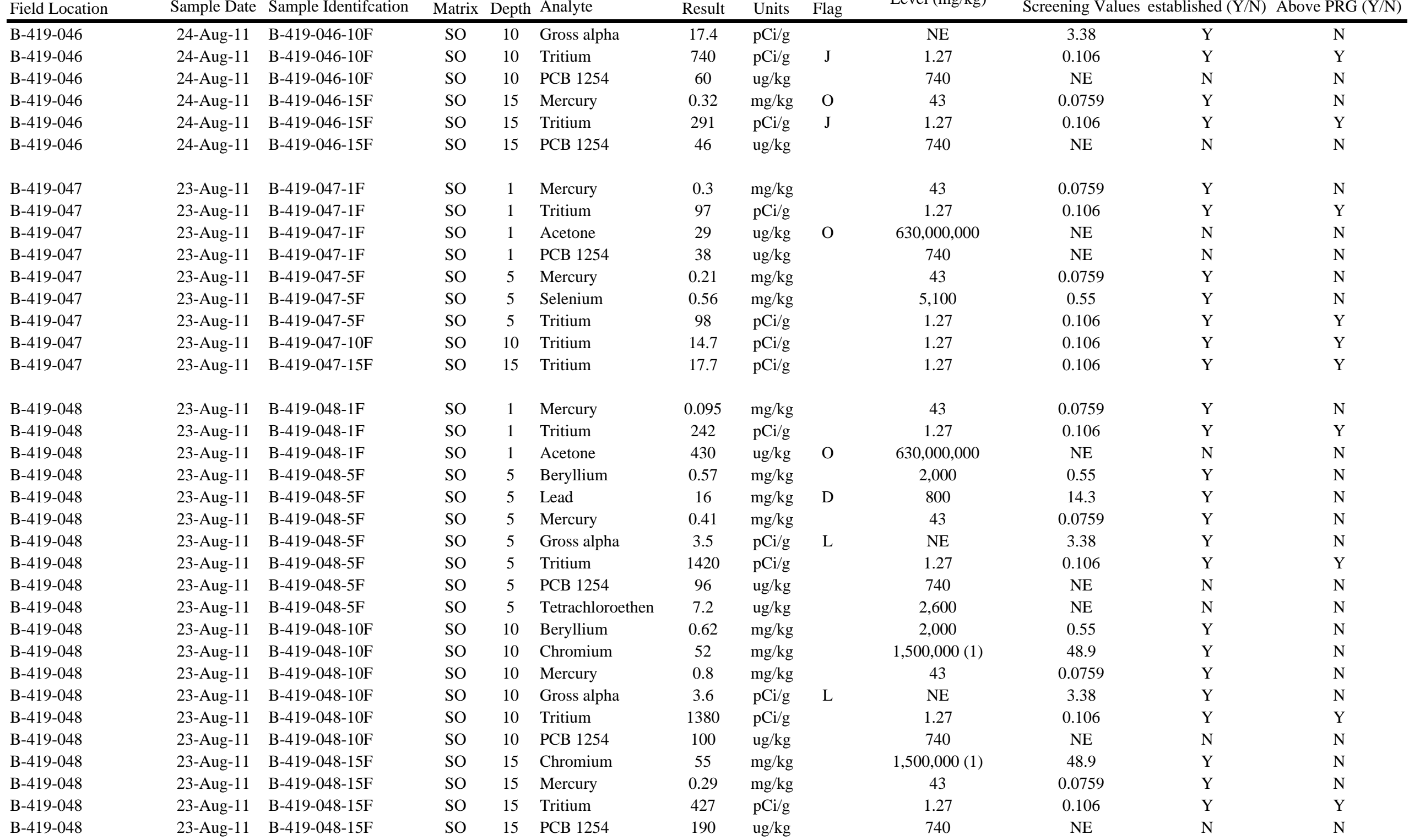


US EPA

Preliminary Bldg

Industrial Screening

Level (mg/kg)

background

\begin{tabular}{|c|c|c|c|c|c|c|c|c|c|c|c|c|}
\hline Field Location & Sample Date & Sample Identifcation & Matrix & Depth & Analyte & Result & Units & Flag & Level (mg/kg) & Screening Values & established $(\mathrm{Y} / \mathrm{N})$ & Above PRG (Y/N) \\
\hline B-419-049 & 22-Aug-11 & B-419-049-1F & SO & 1 & Diesel Fuel & 11 & $\mathrm{mg} / \mathrm{kg}$ & & 180 & $\mathrm{NE}$ & $\mathrm{N}$ & $\mathrm{N}$ \\
\hline B-419-049 & 22-Aug-11 & B-419-049-1F & SO & 1 & Hexavalent & 3.2 & $\mathrm{mg} / \mathrm{kg}$ & $\mathrm{D}$ & 5.6 & 2.2 & $\mathrm{Y}$ & $\mathrm{N}$ \\
\hline B-419-049 & 22-Aug-11 & B-419-049-1F & SO & 1 & Mercury & 0.27 & $\mathrm{mg} / \mathrm{kg}$ & & 43 & 0.0759 & $\mathrm{Y}$ & $\mathrm{N}$ \\
\hline B-419-049 & 22-Aug-11 & B-419-049-1F & SO & 1 & Gross alpha & 3.5 & $\mathrm{pCi} / \mathrm{g}$ & $\mathrm{L}$ & NE & 3.38 & $\mathrm{Y}$ & $\mathrm{N}$ \\
\hline B-419-049 & 22-Aug-11 & B-419-049-1F & SO & 1 & Tritium & 1180 & $\mathrm{pCi} / \mathrm{g}$ & & 1.27 & 0.106 & $\mathrm{Y}$ & $\mathrm{Y}$ \\
\hline B-419-049 & 22-Aug-11 & B-419-049-5F & SO & 5 & Mercury & 0.25 & $\mathrm{mg} / \mathrm{kg}$ & & 43 & 0.0759 & $\mathrm{Y}$ & $\mathrm{N}$ \\
\hline B-419-049 & 22-Aug-11 & B-419-049-5F & SO & 5 & Tritium & 1680 & $\mathrm{pCi} / \mathrm{g}$ & & 1.27 & 0.106 & $\mathrm{Y}$ & $\mathrm{Y}$ \\
\hline B-419-049 & 22-Aug-11 & B-419-049-5F & SO & 5 & РCB 1254 & 81 & ug/kg & & 740 & NE & $\mathrm{N}$ & $\mathrm{N}$ \\
\hline B-419-049 & 22-Aug-11 & B-419-049-10F & SO & 10 & Mercury & 0.27 & $\mathrm{mg} / \mathrm{kg}$ & & 43 & 0.0759 & $\mathrm{Y}$ & $\mathrm{N}$ \\
\hline B-419-049 & 22-Aug-11 & B-419-049-10F & SO & 10 & Tritium & 1740 & $\mathrm{pCi} / \mathrm{g}$ & & 1.27 & 0.106 & $\mathrm{Y}$ & $\mathrm{Y}$ \\
\hline B-419-049 & 22-Aug-11 & B-419-049-10F & SO & 10 & РCB 1254 & 74 & ug/kg & & 740 & $\mathrm{NE}$ & $\mathrm{N}$ & $\mathrm{N}$ \\
\hline B-419-049 & 22-Aug-11 & B-419-049-15F & SO & 15 & Tritium & 1440 & $\mathrm{pCi} / \mathrm{g}$ & & 1.27 & 0.106 & $\mathrm{Y}$ & $\mathrm{Y}$ \\
\hline B-419-050 & 22-Aug-11 & B-419-050-1F & SO & 1 & Beryllium & 1.3 & $\mathrm{mg} / \mathrm{kg}$ & & 2,000 & 0.55 & $\mathrm{Y}$ & $\mathrm{N}$ \\
\hline B-419-050 & 22-Aug-11 & B-419-050-1F & SO & 1 & Lead & 150 & $\mathrm{mg} / \mathrm{kg}$ & $\mathrm{O}$ & 800 & 14.3 & $\mathrm{Y}$ & $\mathrm{N}$ \\
\hline B-419-050 & 22-Aug-11 & B-419-050-1F & SO & 1 & Mercury & 13 & $\mathrm{mg} / \mathrm{kg}$ & $\mathrm{D}$ & 43 & 0.0759 & $\mathrm{Y}$ & $\mathrm{N}$ \\
\hline B-419-050 & 22-Aug-11 & B-419-050-1F & SO & 1 & Chromium & 0.14 & $\mathrm{mg} / \mathrm{L}$ & & NA & NA & NA & NA \\
\hline B-419-050 & 22-Aug-11 & B-419-050-1F & SO & 1 & Cobalt & 0.22 & $\mathrm{mg} / \mathrm{L}$ & & NA & NA & NA & NA \\
\hline B-419-050 & 22-Aug-11 & B-419-050-1F & SO & 1 & Copper & 0.13 & $\mathrm{mg} / \mathrm{L}$ & & NA & NA & NA & NA \\
\hline B-419-050 & 22-Aug-11 & B-419-050-1F & SO & 1 & Lead & 0.15 & $\mathrm{mg} / \mathrm{L}$ & & NA & NA & NA & NA \\
\hline B-419-050 & 22-Aug-11 & B-419-050-1F & SO & 1 & Lead & 0.67 & $\mathrm{mg} / \mathrm{L}$ & & NA & NA & NA & NA \\
\hline B-419-050 & 22-Aug-11 & B-419-050-1F & SO & 1 & Mercury & 0.06 & $\mathrm{mg} / \mathrm{L}$ & & NA & NA & NA & NA \\
\hline B-419-050 & 22-Aug-11 & B-419-050-1F & SO & 1 & Nickel & 0.27 & $\mathrm{mg} / \mathrm{L}$ & B & NA & NA & NA & NA \\
\hline B-419-050 & 22-Aug-11 & B-419-050-1F & SO & 1 & Vanadium & 0.17 & $\mathrm{mg} / \mathrm{L}$ & & NA & NA & NA & NA \\
\hline B-419-050 & 22-Aug-11 & B-419-050-1F & SO & 1 & Zinc & 2.7 & $\mathrm{mg} / \mathrm{L}$ & & NA & NA & NA & NA \\
\hline B-419-050 & 22-Aug-11 & B-419-050-1F & SO & 1 & Gross alpha & 3.6 & $\mathrm{pCi} / \mathrm{g}$ & & $\mathrm{NE}$ & 3.38 & $\mathrm{Y}$ & $\mathrm{N}$ \\
\hline B-419-050 & 22-Aug-11 & B-419-050-1F & SO & 1 & Tritium & 1330 & $\mathrm{pCi} / \mathrm{g}$ & & 1.27 & 0.106 & $\mathrm{Y}$ & $\mathrm{Y}$ \\
\hline B-419-050 & 22-Aug-11 & B-419-050-1F & SO & 1 & РCB 1248 & 280 & ug/kg & $\mathrm{D}$ & 740 & NE & $\mathrm{N}$ & $\mathrm{N}$ \\
\hline B-419-050 & 22-Aug-11 & B-419-050-DUP-1.5F & SO & 1.5 & Beryllium & 1.7 & $\mathrm{mg} / \mathrm{kg}$ & & 2,000 & 0.55 & $\mathrm{Y}$ & $\mathrm{N}$ \\
\hline B-419-050 & 22-Aug-11 & B-419-050-DUP-1.5F & SO & 1.5 & Lead & 300 & $\mathrm{mg} / \mathrm{kg}$ & $\mathrm{O}$ & 800 & 14.3 & $\mathrm{Y}$ & $\mathrm{N}$ \\
\hline B-419-050 & 22-Aug-11 & B-419-050-DUP-1.5F & SO & 1.5 & Mercury & 22 & $\mathrm{mg} / \mathrm{kg}$ & $\mathrm{D}$ & 43 & 0.0759 & $\mathrm{Y}$ & $\mathrm{N}$ \\
\hline B-419-050 & 22-Aug-11 & B-419-050-DUP-1.5F & SO & 1.5 & Tritium & 451 & $\mathrm{pCi} / \mathrm{g}$ & & 1.27 & 0.106 & $\mathrm{Y}$ & $\mathrm{Y}$ \\
\hline B-419-050 & 22-Aug-11 & B-419-050-5F & SO & 5 & Gross alpha & 4 & $\mathrm{pCi} / \mathrm{g}$ & & NE & 3.38 & $\mathrm{Y}$ & $\mathrm{N}$ \\
\hline B-419-050 & 22-Aug-11 & B-419-050-5F & SO & 5 & Tritium & 1130 & $\mathrm{pCi} / \mathrm{g}$ & & 1.27 & 0.106 & $\mathrm{Y}$ & $\mathrm{Y}$ \\
\hline
\end{tabular}


US EPA

Preliminary Bldg

Industrial Screening

Field Location

Sample Date Sample Identifcation

Matrix Depth Analyte 22-Aug-11 B-419-050-10F 22-Aug-11 B-419-050-10F 22-Aug-11 B-419-050-14.5F 22-Aug-11 B-419-050-14.5F

$\begin{array}{cclcc}\text { SO } & 10 & \text { Mercury } & 0.2 & \mathrm{mg} / \mathrm{kg} \\ \text { SO } & 10 & \text { Tritium } & 1430 & \mathrm{pCi} / \mathrm{g} \\ \text { SO } & 14.5 & \text { Mercury } & 0.09 & \mathrm{mg} / \mathrm{kg} \\ \text { SO } & 14.5 & \text { Tritium } & 1350 & \mathrm{pCi} / \mathrm{g}\end{array}$

23-Aug-11 B-419-051-1F 23-Aug-11 B-419-051-1F 23-Aug-11 B-419-051-5F 23-Aug-11 B-419-051-5F 23-Aug-11 B-419-051-10F 23-Aug-11 B-419-051-10F 23-Aug-11 B-419-051-15F 23-Aug-11 B-419-051-15F

19-Sep-10 B-419-101-7F 19-Sep-10 B-419-101-7F 19-Sep-10 B-419-101-10F 19-Sep-10 B-419-101-10F

$\begin{array}{lllll}\text { SO } & 1 & \text { Tritium } & 9.4 & \mathrm{pCi} / \mathrm{g}\end{array}$

$\begin{array}{lllll}\mathrm{SO} & 1 & \text { Tetrachloroethen } & 4.4 & \mathrm{ug} / \mathrm{kg}\end{array}$

B-419-05

B-419-051

B-419-05

B-419-05

B-419-05

B-419-101

B-419-101

B-419-10

B-419-101

19-Sep-10 B-419-102-DUP-1F

19-Sep-10 B-419-102-1F

19-Sep-10 B-419-102-DUP-1F

19-Sep-10 B-419-102-1F

19-Sep-10 B-419-102-3F

B-419-102

B-419-103

B-419-103

B-419-103

B-419-103

B-419-103

B-419-104

B-419-104

B-419-104

B-419-104

B-419-104

B-419-104
21-Sep-10 B-419-103-7F

21-Sep-10 B-419-103-10F

21-Sep-10 B-419-103-10F

21-Sep-10 B-419-103-25F

21-Sep-10 B-419-103-30F

21-Sep-10 B-419-104-DUP-7F

21-Sep-10 B-419-104-7F

21-Sep-10 B-419-104-10F

21-Sep-10 B-419-104-10F

21-Sep-10 B-419-104-25F

21-Sep-10 B-419-104-30F
SO 5 Mercury $\quad 0.17 \quad \mathrm{mg} / \mathrm{kg}$

$\begin{array}{lllll}\mathrm{SO} & 5 & \text { Tritium } & 99 & \mathrm{pCi} / \mathrm{g}\end{array}$

SO 10 Mercury $\quad 0.11 \quad \mathrm{mg} / \mathrm{kg}$

$\begin{array}{lllll}\text { SO } & 10 & \text { Tritium } & 17.7 & \mathrm{pCi} / \mathrm{g}\end{array}$

SO 15 Mercury $\quad 0.087 \quad \mathrm{mg} / \mathrm{kg}$

SO 15 Tritium $5.33 \quad \mathrm{pCi} / \mathrm{g}$

$\begin{array}{llllll}\text { SO } & 7 & \text { Mercury } & 0.093 \quad \mathrm{mg} / \mathrm{kg} & \text { B }\end{array}$

SO $\quad 7$ Tritium

SO 10 Mercury

SO 10 Tritium

$228 \mathrm{pCi} / \mathrm{g}$

$0.39 \mathrm{mg} / \mathrm{kg} \quad \mathrm{B}$

$1130 \mathrm{pCi} / \mathrm{g}$

SO 1 Diesel Fuel

SO 1 Diesel Fuel

44

$\quad 120 \quad \mathrm{mg} / \mathrm{kg}$

Methylene chlorid 430 ug/L DB

Methylene chlorid $440 \quad$ ug/L $\quad$ DB

3 Diesel Fuel

$9.1 \mathrm{mg} / \mathrm{kg}$

\section{$\begin{array}{llllll}\text { SO } & 7 & \text { Tritium } & 0.207 & \mathrm{pCi} / \mathrm{g} & \mathrm{J}\end{array}$}

SO 10 Mercury

SO 10 Tritium

SO 25 Trichloroethene

SO 30 Diesel Fuel

$\begin{array}{ccc}0.19 & \mathrm{mg} / \mathrm{kg} & \mathrm{B} \\ 1.8 & \mathrm{pCi} / \mathrm{g} & \mathrm{J}\end{array}$

11

$\mathrm{ug} / \mathrm{kg}$

$\mathrm{mg} / \mathrm{kg}$

\section{B}

$\begin{array}{ccc}0.1 & \mathrm{mg} / \mathrm{kg} & \text { B } \\ 0.74 & \mathrm{pCi} / \mathrm{g} & \mathrm{J} \\ 0.086 & \mathrm{mg} / \mathrm{kg} & \text { B } \\ 0.89 & \mathrm{pCi} / \mathrm{g} & \mathrm{J} \\ 16 & \mathrm{mg} / \mathrm{kg} & \text { B } \\ 9.9 & \mathrm{mg} / \mathrm{kg} & \text { B }\end{array}$

Level (mg/kg)

background

Screening Values established (Y/N) Above PRG (Y/N)

$\begin{array}{cccc}43 & 0.0759 & \mathrm{Y} & \mathrm{N} \\ 1.27 & 0.106 & \mathrm{Y} & \mathrm{Y} \\ 43 & 0.0759 & \mathrm{Y} & \mathrm{N} \\ 1.27 & 0.106 & \mathrm{Y} & \mathrm{Y}\end{array}$

1.27

2,600

43

1.27

1.27

43

1.27

43

1.27

1.27

180

180

NA

NA

180

1.27

43

1.27

6,400

180

43
1.27
43
1.27
180
180

$\begin{array}{cc}0.106 & \mathrm{Y} \\ \mathrm{NE} & \mathrm{N} \\ 0.0759 & \mathrm{Y} \\ 0.106 & \mathrm{Y} \\ 0.0759 & \mathrm{Y} \\ 0.106 & \mathrm{Y} \\ 0.0759 & \mathrm{Y} \\ 0.106 & \mathrm{Y} \\ & \\ 0.0759 & \mathrm{Y} \\ 0.106 & \mathrm{Y} \\ 0.0759 & \mathrm{Y} \\ 0.106 & \mathrm{Y} \\ & \\ \mathrm{NE} & \mathrm{N} \\ \mathrm{NE} & \mathrm{N} \\ \mathrm{NA} & \mathrm{N} \\ \mathrm{NA} & \mathrm{N} \\ \mathrm{NE} & \mathrm{N} \\ & \\ 0.106 & \mathrm{Y} \\ 0.0759 & \mathrm{Y} \\ 0.106 & \mathrm{Y} \\ \mathrm{NE} & \mathrm{N} \\ \mathrm{NE} & \mathrm{N} \\ 0.0759 & \mathrm{Y} \\ 0.106 & \mathrm{Y} \\ 0.0759 & \mathrm{Y} \\ 0.106 & \mathrm{Y} \\ \mathrm{NE} & \end{array}$


US EPA

Preliminary Bldg

Industrial Screening 419 Background

background

\begin{tabular}{|c|c|c|c|c|c|c|c|c|c|c|c|c|}
\hline Field Location & Sample Date & Sample Identifcation & Matrix & Depth & Analyte & Result & Units & Flag & Level (mg/kg) & Screening Values & established (Y/N) & Above PRG (Y/N) \\
\hline B-419-105 & 22-Sep-10 & B-419-105-25F & SO & 25 & $\operatorname{Bis}(2-$ & 420 & $\mathrm{ug} / \mathrm{kg}$ & & 120,000 & $\mathrm{NE}$ & $\mathrm{N}$ & $\mathrm{N}$ \\
\hline B-419-106 & 22-Sep-10 & B-419-106-7F & SO & 7 & Mercury & 0.084 & $\mathrm{mg} / \mathrm{kg}$ & & 43 & 0.0759 & $\mathrm{Y}$ & $\mathrm{N}$ \\
\hline B-419-R124-001 & 19-Sep-11 & B-419-R124-001-3F & SO & 3 & Beryllium & 0.76 & $\mathrm{mg} / \mathrm{kg}$ & & 2,000 & 0.55 & $\mathrm{Y}$ & $\mathrm{N}$ \\
\hline B-419-R124-001 & 19-Sep-11 & B-419-R124-001-3F & SO & 3 & Diesel Fuel & 5.7 & $\mathrm{mg} / \mathrm{kg}$ & & 180 & NE & $\mathrm{N}$ & $\mathrm{N}$ \\
\hline B-419-R124-001 & 19-Sep-11 & B-419-R124-001-3F & SO & 3 & Mercury & 4.3 & $\mathrm{mg} / \mathrm{kg}$ & $\mathrm{D}$ & 43 & 0.0759 & $\mathrm{Y}$ & $\mathrm{N}$ \\
\hline B-419-R124-001 & 19-Sep-11 & B-419-R124-001-3F & SO & 3 & Molybdenum & 2.1 & $\mathrm{mg} / \mathrm{kg}$ & & 5,100 & 1.1 & $\mathrm{Y}$ & $\mathrm{N}$ \\
\hline B-419-R124-001 & 19-Sep-11 & B-419-R124-001-3F & SO & 3 & Barium & 1 & $\mathrm{mg} / \mathrm{L}$ & & NA & NA & NA & NA \\
\hline B-419-R124-001 & 19-Sep-11 & B-419-R124-001-3F & SO & 3 & Barium & 8.2 & $\mathrm{mg} / \mathrm{L}$ & B & NA & NA & NA & NA \\
\hline B-419-R124-001 & 19-Sep-11 & B-419-R124-001-3F & SO & 3 & Chromium & 0.11 & $\mathrm{mg} / \mathrm{L}$ & & NA & NA & NA & NA \\
\hline B-419-R124-001 & 19-Sep-11 & B-419-R124-001-3F & SO & 3 & Cobalt & 0.34 & $\mathrm{mg} / \mathrm{L}$ & & NA & NA & NA & NA \\
\hline B-419-R124-001 & 19-Sep-11 & B-419-R124-001-3F & SO & 3 & Copper & 0.34 & $\mathrm{mg} / \mathrm{L}$ & & NA & NA & NA & NA \\
\hline B-419-R124-001 & 19-Sep-11 & B-419-R124-001-3F & SO & 3 & Lead & 0.094 & $\mathrm{mg} / \mathrm{L}$ & & NA & NA & NA & NA \\
\hline B-419-R124-001 & 19-Sep-11 & B-419-R124-001-3F & SO & 3 & Mercury & 0.015 & $\mathrm{mg} / \mathrm{L}$ & & NA & NA & NA & NA \\
\hline B-419-R124-001 & 19-Sep-11 & B-419-R124-001-3F & SO & 3 & Nickel & 0.44 & $\mathrm{mg} / \mathrm{L}$ & & NA & NA & NA & NA \\
\hline B-419-R124-001 & 19-Sep-11 & B-419-R124-001-3F & SO & 3 & Americium 241 & 0.159 & $\mathrm{pCi} / \mathrm{g}$ & B & 4.82 & 0.062 & $\mathrm{Y}$ & $\mathrm{N}$ \\
\hline B-419-R124-001 & 19-Sep-11 & B-419-R124-001-3F & SO & 3 & Gross alpha & 21.2 & $\mathrm{pCi} / \mathrm{g}$ & & NE & 3.38 & $\mathrm{Y}$ & $\mathrm{N}$ \\
\hline B-419-R124-001 & 19-Sep-11 & B-419-R124-001-3F & SO & 3 & Gross beta & 28.1 & $\mathrm{pCi} / \mathrm{g}$ & & $\mathrm{NE}$ & 4.47 & $\mathrm{Y}$ & $\mathrm{N}$ \\
\hline B-419-R124-001 & 19-Sep-11 & B-419-R124-001-3F & SO & 3 & Lead 212 & 0.4 & $\mathrm{pCi} / \mathrm{g}$ & & 5,330 & 0.938 & $\mathrm{Y}$ & $\mathrm{N}$ \\
\hline B-419-R124-001 & 19-Sep-11 & B-419-R124-001-3F & SO & 3 & Plutonium $239+2 \measuredangle$ & 0.64 & $\mathrm{pCi} / \mathrm{g}$ & $\mathrm{O}$ & 12.5 & 0.046 & $\mathrm{Y}$ & $\mathrm{N}$ \\
\hline B-419-R124-001 & 19-Sep-11 & B-419-R124-001-3F & SO & 3 & Potassium 40 & 10.8 & $\mathrm{pCi} / \mathrm{g}$ & & 0.265 & 18.4 & $\mathrm{Y}$ & $\mathrm{Y}$ \\
\hline B-419-R124-001 & 19-Sep-11 & B-419-R124-001-3F & SO & 3 & Thorium 228 & 0.6 & $\mathrm{pCi} / \mathrm{g}$ & & 109 & 0.991 & $\mathrm{Y}$ & $\mathrm{N}$ \\
\hline B-419-R124-001 & 19-Sep-11 & B-419-R124-001-3F & SO & 3 & Thorium 230 & 0.56 & $\mathrm{pCi} / \mathrm{g}$ & & 18 & 0.957 & $\mathrm{Y}$ & $\mathrm{N}$ \\
\hline B-419-R124-001 & 19-Sep-11 & B-419-R124-001-3F & SO & 3 & Thorium 232 & 0.56 & $\mathrm{pCi} / \mathrm{g}$ & & 17 & 0.983 & $\mathrm{Y}$ & $\mathrm{N}$ \\
\hline B-419-R124-001 & 19-Sep-11 & B-419-R124-001-3F & SO & 3 & Thorium 234 & 30 & $\mathrm{pCi} / \mathrm{g}$ & & 2,750 & 2.44 & $\mathrm{Y}$ & $\mathrm{N}$ \\
\hline B-419-R124-001 & 19-Sep-11 & B-419-R124-001-3F & SO & 3 & Tritium & 2.2 & $\mathrm{pCi} / \mathrm{g}$ & & 1.27 & 0.106 & $\mathrm{Y}$ & $\mathrm{Y}$ \\
\hline B-419-R124-001 & 19-Sep-11 & B-419-R124-001-3F & SO & 3 & Uranium 234 and & 18.4 & $\mathrm{pCi} / \mathrm{g}$ & & 25.5 & 0.76 & $\mathrm{Y}$ & $\mathrm{N}$ \\
\hline B-419-R124-001 & 19-Sep-11 & B-419-R124-001-3F & SO & 3 & Uranium 235 and & 1.39 & $\mathrm{pCi} / \mathrm{g}$ & & 31.3 & 0.0904 & $\mathrm{Y}$ & $\mathrm{N}$ \\
\hline B-419-R124-001 & 19-Sep-11 & B-419-R124-001-3F & SO & 3 & Uranium 238 & 46 & $\mathrm{pCi} / \mathrm{g}$ & & 33 & 0.717 & $\mathrm{Y}$ & $\mathrm{Y}$ \\
\hline B-419-R124-001 & 19-Sep-11 & B-419-R124-001-3F & SO & 3 & Acetone & 100 & $\mathrm{ug} / \mathrm{kg}$ & & $630,000,000$ & $\mathrm{NE}$ & $\mathrm{N}$ & $\mathrm{N}$ \\
\hline B-419-R124-001 & 19-Sep-11 & B-419-R124-001-5F & SO & 5 & Mercury & 0.14 & $\mathrm{mg} / \mathrm{kg}$ & & 43 & 0.0759 & $\mathrm{Y}$ & $\mathrm{N}$ \\
\hline
\end{tabular}


US EPA

Preliminary Bldg

Industrial Screening

background

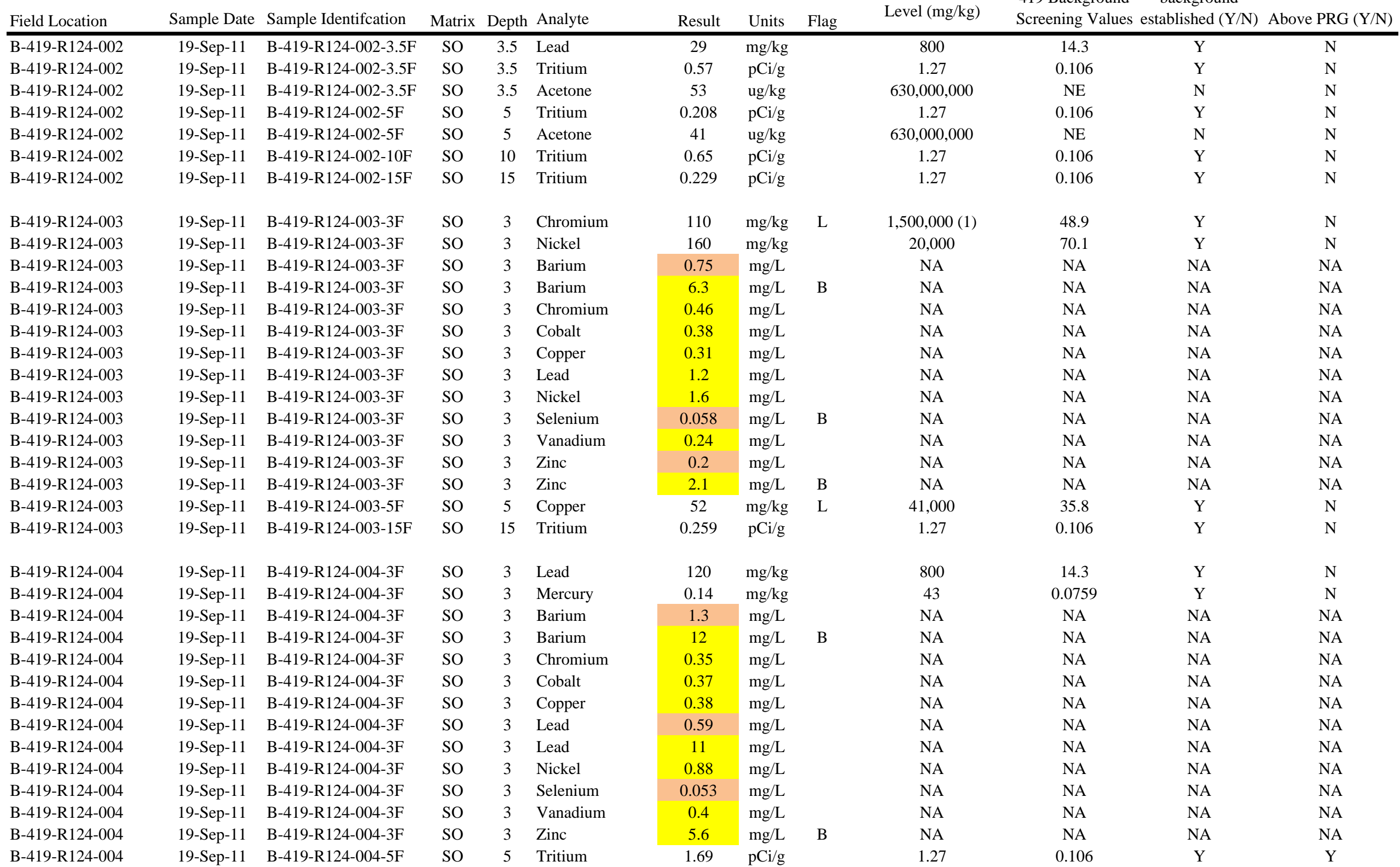


US EPA

Industrial Screening

Level (mg/kg)

Preliminary Bldg

419 Backoroun

background

Field Location

Sample Date Sample Identifcation

Matrix Depth Analyte

Result

Units Flag

Screening Values established (Y/N) Above PRG (Y/N)

B-419-R124-004

B-419-R124-004

19-Sep-11 B-419-R124-004-5F

SO 5 Acetone

19-Sep-11 B-419-R124-004-10F

19-Sep-11 B-419-R124-004-15

$630,000,000$

NE
0.106

0.106

$0.208 \quad \mathrm{pCi} / \mathrm{g}$

1.27

0.106

$\mathrm{N}$
$\mathrm{Y}$

$\mathrm{Y}$
$\mathrm{Y}$

$\mathrm{N}$

N 
US EPA

Industrial Screen

Level (mg/kg)

Preliminary Bldg

Result Units Flag
419 Background background

Screening Values established (Y/N) Above PRG (Y/N)

Notes:

Data presented are detects in soil above background. If a background number has not been established for an analyte that analyte is listed.

Industrial screening levels are United States Environmental Protection Regional screening levels for all analytes except for radionuclides and diesel.

Radionuclides were screened against USEPA Composite Worker Soil Preliminary Remediation Goals.

Diesel and gasoling range analytes were compared to the RWQCB Environmental Screening Levels for shallow soil and non drinking water conditions were used.

(1) Chromium is assumed to be Chromium III because Hexavalent Chromium was not detected in the sample.

= Analyte analyzed by STLC

= Analyte analyzed by TCLP

$\mathrm{AQ}=$ Aqueous matrix

$\mathrm{OT}=$ concrete matrix

$\mathrm{SO}=$ soil matrix

ESL=Regional Water Quality Control Board Environmental Sreening Value

MCL = United States Environmental Protection Agency maximum contaminant level

$\mathrm{NE}=$ not established

action level = United States Environmental Protection Agency action level

QC Flag = Quality Control (QC) flags:

$\mathrm{B}=$ analyte found in method blank 


\section{The Development of}

Project-Specific Background

Levels for Soil 


\section{B419 Background Screening Values (Background Threshold Values)}

March 2012

For use in the B419 Project LLNL has developed a set of background threshold values (EPA 2007a, 2007b) for total metals and radiological isotopes in soil. The background screening values are displayed in the attached table. There were three steps to this process: (1) identify locations considered to be represent background for the Livermore Site; (2) collect and analyze samples; and (3) calculate background threshold values (BTVs; also referred to as screening values) from the analytical results.

To determine how many samples to collect, LLNL followed the MARSSIM guidance for a Wilcoxon Rank Sum Test or Sign Test, with $\Delta / \sigma=1$ and $5 \%$ for both the Type I and Type II errors. This means that if the B419 site is more than one standard deviation above or below the site background those statistical tests would have a high probability (95\%) of making the correct decision. These choices indicate that the number of measurements is $n=32$, in both the site and the background (MARSSIM Table I.2b). Therefore, at least 32 background samples are required. To account for the possibility of lost samples, an additional three sample locations were identified (i.e., to allow for up to $10 \%$ lost samples). Therefore, a minimum of 35 randomly selected locations was required. The final sample locations were identified as follows:

1. Two hundred locations within the LLNL perimeter were generated using a random number generator.

2. Using a basemap showing locations of buildings, roads, etc., the two hundred random points were screened, and those that were inside buildings were removed.

3. The remaining 166 locations were reviewed by staff from the Environmental Restoration Division CERCLA remediation effort, and those considered to be inappropriate for use as background were flagged. Thirty-three of the 166 were eliminated this review, leaving 133 potential sampling locations.

The remaining 133 locations were then available for background sampling. Due to potential inaccuracies in the basemap it was expected that some of the 133 locations could be in paved areas or otherwise inaccessible. If there was accessible soil within 25 feet of such a location, the location was adjusted. Otherwise the location was discarded and the next location in the list was used. Since the number of discarded locations was unknown in advance of the sampling effort, the first 50 of the 133 locations, shown in Figure 1, were given to the sampling team. The remaining 83 locations were held in reserve, and it turned out to be unnecessary to use any of them. The sampling team was directed to go through the list sequentially, in order to preserve the randomness of the locations.

One composite sample was collected using a hand auger from each location at a depth between 6 inches to 2 feet below grade. Each sample was analyzed for TTLC metals and 
radioactivity, including gross alpha, gross beta, tritium, alpha spectroscopy, gamma spectroscopy, Carbon 14, Strontium 90, and Technetium 99).

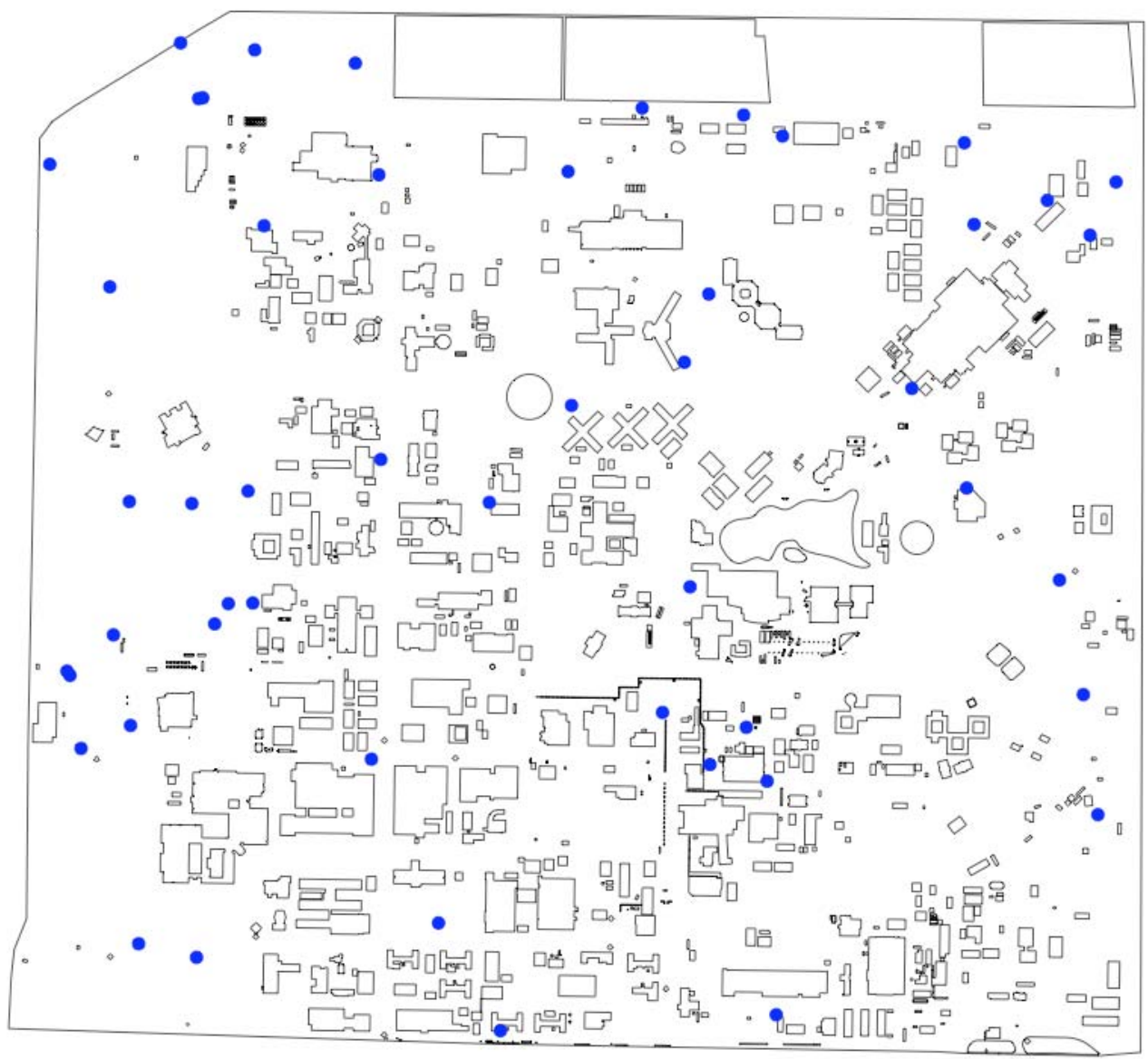

Figure 1. Fifty randomly selected sample locations on the Livermore Site.

Subsequently, LLNL chose to compare each B419 Site sample individually with background. As stated in EPA 2007b (page v), “... upper limits based upon background (or reference) data are used as estimates of BTVs, compliance limits $(C L)$, or notto-exceed values. These upper limits are often used in site (point-by-point) versus background comparison evaluations.” BTVs are designed to represent a value that should rarely be exceeded by samples from background.

Specifically, LLNL chose to use the upper prediction limit (UPL) method for the normal distribution from EPA 2007b (Eq. 3-10 in Section 3.6.1 with $\alpha=0.005$ ) to calculate BTVs. Samples from background should exceed such a BTV at a rate of approximately 1 in 200. The key to successful use of the UPL for a BTV is to determine whether the 
statistical model that underlies the UPL method fits the data. If it does not, then the exceedance rate in actual use could be substantially different from the intended rate.

LLNL first reviewed results for outliers and the number of results reported by the analytical laboratory as above the contract reporting limit (referred to as ">CRL"). One or two high outliers were excluded from four analytes. With one exception, the metals were either all $>$ CRL, or did not have enough results $>$ CRL for statistical calculations. The exception, mercury, had 21 out of 33 results >CRL (after excluding two outliers), and this was considered sufficient for statistical calculations. For radiological isotopes LLNL required the laboratory to report estimated concentrations based on the underlying count data, even when the result was $<$ CRL. These estimated concentrations were used.

After these reviews the statistical distribution of the results was examined. All of the analytes' probability plots indicated that the normal distribution was a good fit, so the UPL was calculated as described above.

\section{References}

EPA 2007a. ProUCL Version 4.0 User Guide, EPA/600/R-07/038, 2007.

EPA 2007b. ProUCL Version 4.0 Technical Guide, EPA/600/R-07/041, 2007.

For reference, here is a quote of the first three paragraphs of the executive summary of EPA 2007b (page v).

\section{Executive Summary}

Statistical inference, including both estimation and hypotheses testing approaches, is routinely used to:

1. Estimate environmental parameters of interest, such as exposure point concentration (EPC) terms, not-to-exceed values, and background level threshold values (BTVs) for contaminants of potential concern (COPC),

2. Identify areas of concern (AOC) at a contaminated site,

3. Compare contaminant concentrations found at two or more AOCs of a contaminated site,

4. Compare contaminant concentrations found at an AOC with background or reference area contaminant concentrations, and

5. Compare site concentrations with a cleanup standard to verify the attainment of cleanup standards. 
Several exposure and risk management and cleanup decisions in support of United States Environmental Protection Agency (EPA) projects are often made based upon the mean concentrations of the COPCs. A 95\% upper confidence limit (UCL95) of the unknown population (e.g., an AOC) arithmetic mean (AM), $\mu 1$, can be used to:

- Estimate the EPC term of the AOC under investigation,

- Determine the attainment of cleanup standards,

- Compare site mean concentrations with reference area mean concentrations, and

- Estimate background level mean contaminant concentrations. The background mean contaminant concentration level may be used to compare the mean of an area of concern. It should be noted that it is not appropriate to compare individual point-by-point site observations with the background mean concentration level.

It is important to compute a reliable and stable UCL95 of the population mean using the available data. The UCL95 should approximately provide the $95 \%$ coverage for the unknown population mean, $\mu 1$. Based upon the available background data, it is equally important to compute reliable and stable upper percentiles, upper prediction limits (UPLs), or upper tolerance limits (UTLs). These upper limits based upon background (or reference) data are used as estimates of BTVs, compliance limits $(C L)$, or not-to- exceed values. These upper limits are often used in site (point-by-point) versus background comparison evaluations. 


\begin{tabular}{|c|c|c|}
\hline Description & ParType & Screening value \\
\hline Antimony & Metal & 2.2 \\
\hline Arsenic & Metal & 7.59 \\
\hline Barium & Metal & 332 \\
\hline Beryllium & Metal & 0.55 \\
\hline Cadmium & Metal & 0.55 \\
\hline Chromium & Metal & 48.9 \\
\hline Cobalt & Metal & 16.7 \\
\hline Copper & Metal & 35.8 \\
\hline Hexavalent Chromium & Metal & 2.2 \\
\hline Lead & Metal & 14.3 \\
\hline Mercury & Metal & 0.0759 \\
\hline Molybdenum & Metal & 1.1 \\
\hline Nickel & Metal & 70.1 \\
\hline Selenium & Metal & 0.55 \\
\hline Silver & Metal & 1.1 \\
\hline Thallium & Metal & 1.1 \\
\hline Vanadium & Metal & 43.7 \\
\hline Zinc & Metal & 69.8 \\
\hline Actinium 228 & Radiological & 1.5 \\
\hline Aluminum 26 & Radiological & 0.133 \\
\hline Am $241 \mathrm{GS}$ & Radiological & 0.539 \\
\hline Americium 241 & Radiological & 0.062 \\
\hline Antimony 124 & Radiological & 0.145 \\
\hline Antimony 125 & Radiological & 0.35 \\
\hline Beryllium 7 & Radiological & 1.15 \\
\hline Bismuth 212 & Radiological & 2.52 \\
\hline Bismuth 214 & Radiological & 0.912 \\
\hline Carbon 14 & Radiological & 10.2 \\
\hline Cerium 139 & Radiological & 0.0777 \\
\hline Cerium 144 & Radiological & 0.626 \\
\hline Cesium 134 & Radiological & 0.189 \\
\hline Cesium 137 & Radiological & 0.16 \\
\hline Chromium 51 & Radiological & 0.957 \\
\hline Cobalt 56 & Radiological & 0.428 \\
\hline Cobalt 57 & Radiological & 0.0547 \\
\hline Cobalt 58 & Radiological & 0.181 \\
\hline Cobalt 60 & Radiological & 0.154 \\
\hline Curium 242 & Radiological & 0.0143 \\
\hline Curium 244 & Radiological & 0.0269 \\
\hline Europium 152 & Radiological & 0.88 \\
\hline Europium 154 & Radiological & 0.852 \\
\hline Europium 155 & Radiological & 0.34 \\
\hline Gross alpha & Radiological & 3.38 \\
\hline Gross beta & Radiological & 4.47 \\
\hline lodine 131 & Radiological & 0.395 \\
\hline Iron 59 & Radiological & 0.399 \\
\hline
\end{tabular}




\begin{tabular}{|l|l|r|}
\hline Lead 212 & Radiological & 0.938 \\
\hline Lead 214 & Radiological & 0.844 \\
\hline Manganese 54 & Radiological & 0.159 \\
\hline Niobium 94 & Radiological & 0.137 \\
\hline Niobium 95 & Radiological & 0.169 \\
\hline Plutonium 238 & Radiological & 0.0355 \\
\hline Plutonium 239+240 & Radiological & 0.046 \\
\hline Potassium 40 & Radiological & 18.4 \\
\hline Protactinium 234m & Radiological & 29.6 \\
\hline Ruthenium 106 & Radiological & 1.25 \\
\hline Scandium 46 & Radiological & 0.134 \\
\hline Silver 110m & Radiological & 0.161 \\
\hline Sodium 22 & Radiological & 0.188 \\
\hline Strontium 90 & Radiological & 0.264 \\
\hline Technetium 99 & Radiological & 0.647 \\
\hline Thallium 208 & Radiological & 0.371 \\
\hline Thorium 227 & Radiological & 1.05 \\
\hline Thorium 228 & Radiological & 0.991 \\
\hline Thorium 230 & Radiological & 0.957 \\
\hline Thorium 232 & Radiological & 0.983 \\
\hline Thorium 234 & Radiological & 2.44 \\
\hline Tritium & Radiological & 0.106 \\
\hline Uranium 234 and Uranium 23 & Radiological & 0.76 \\
\hline Uranium 235 & Radiological & 0.645 \\
\hline Uranium 235 and Uranium 23 & Radiological & 0.0904 \\
\hline Uranium 238 & Radiological & 0.717 \\
\hline Zinc 65 & Radiological & 0.261 \\
\hline
\end{tabular}

